\title{
Fano Symmetric Varieties with Low Rank
}

\author{
by
}

Alessandro Ruzzi

\begin{abstract}
The symmetric projective varieties of rank one are all smooth and Fano by a classical result of Akhiezer. We classify the locally factorial (respectively smooth) projective symmetric $G$-varieties of rank 2 that are Fano. When $G$ is semisimple we also classify the locally factorial (respectively smooth) projective symmetric $G$-varieties of rank 2 that are only quasi-Fano. Moreover, we classify the Fano symmetric $G$-varieties of rank 3 obtainable from a wonderful variety by a sequence of blow-ups along $G$-stable varieties. Finally, we classify the Fano symmetric varieties of arbitrary rank that are obtainable from a wonderful variety by a sequence of blow-ups along closed orbits.
\end{abstract}

2010 Mathematics Subject Classification: Primary 14J45; Secondary 14M27.

Keywords: Fano varieties, symmetric varieties.

A Gorenstein (projective) normal algebraic variety $X$ over $\mathbb{C}$ is called a Fano variety if the anticanonical divisor is ample. The Fano surfaces are classically called Del Pezzo surfaces. The importance of Fano varieties in the theory of higher dimensional varieties is similar to the importance of Del Pezzo surfaces in the theory of surfaces. Moreover Mori's program predicts that every uniruled variety is birational to a fiberspace whose general fiber is a Fano variety (with terminal singularities).

Let $\theta$ be an involution of a reductive group $G$ (over $\mathbb{C}$ ) and let $H$ be a closed subgroup of $G$ such that $G^{\theta} \subset H \subset N_{G}\left(G^{\theta}\right)$. A normal $G$-variety with an open orbit isomorphic to $G / H$ is called a symmetric variety. Symmetric varieties are a generalization of toric varieties. The toric smooth Fano varieties with rank at most four have been classified. By Theorem 4.2 of [AlBr04], there are finitely many Fano smooth symmetric varieties with a fixed open orbit. In [Ru07] we have classified the smooth compact symmetric varieties with Picard number one and $G$ semisimple,

Communicated by S. Mukai. Received October 25, 2010. Revised February 8, 2011.

A. Ruzzi: Dipartimento di Matematica "G. Castelnuovo", "Sapienza" Università di Roma, Piazzale Aldo Moro 5, 00185 Roma, Italy;

e-mail: alessandroruzzi78@gmail.com 
while in [Ru10] we have given an explicit geometrical description of such varieties; they are automatically Fano.

In this work, we want to classify the Fano symmetric varieties with low rank (and $G$ semisimple). First, we consider a special case of arbitrary rank. We say that a variety $X$ is quasi $\mathbb{Q}$-Fano if $-K_{X}$ is a nef and big $\mathbb{Q}$-divisor. For any fixed open orbit $G / H$ with $G$ semisimple, there is a unique maximal compactification among those that have only one closed orbit. Such a variety is called the standard compactification. Vice versa, if such a compactification exists, then we can suppose that $G$ is semisimple. If the standard compactification is smooth, it is called the wonderful compactification; this is the case, for example, if $H=N_{G}\left(G^{\theta}\right)$ (see [dCoPr83, Theorem 3.1]). We prove that the standard symmetric varieties are all quasi $\mathbb{Q}$-Fano and we describe when they are Fano. In particular, we prove:

Theorem A. Let $X$ be a wonderful symmetric variety. Then:

- The anticanonical divisor of $X$ is always nef and big.

- If $X$ is not a Fano variety and $G$ is simple, then $H=N\left(G^{\theta}\right)$ and there is a maximal torus of $G$ over which $\theta$ acts as the inverse map.

We also determine the Fano symmetric varieties obtainable from a wonderful one by a sequence of blow-ups along closed orbits. In particular, we prove:

Theorem B. Let $X$ be a Fano symmetric variety obtainable from a wonderful one $X_{0}$ by a sequence of blow-ups along closed orbits. Then $X$ is either $X_{0}$ or the blow-up of $X_{0}$ along the unique closed orbit.

Next we consider symmetric varieties of rank at most three. The rank of a symmetric variety $X$ is the maximal codimension of an orbit under the action of the unipotent radical of any Borel subgroup of $G$. The symmetric varieties with rank one are all wonderful; moreover one can show that they are isomorphic, under the action of $\operatorname{Aut}^{0}(X)$, either to a projective homogeneous variety $G / P$ with $P$ maximal, or to $\mathbb{P}^{n} \times \mathbb{P}^{n}$ (see [A83]). Thus they are all Fano.

We classify all the locally factorial (resp. smooth) Fano symmetric varieties of rank 2. When $G$ is semisimple, we also classify the locally factorial (resp. smooth) symmetric varieties that are only quasi-Fano. In the proof of this result we obtain a classification of the toroidal Fano varieties of rank 2 with $G$ semisimple (without the regularity assumption). To state the previous results we need the theory of colored fans. However, if we suppose that there is a wonderful compactification $X_{0}$ and that $X$ is toroidal (i.e. $X$ dominates $X_{0}$ ), then the previous theorems have a more elementary form. Supposing the existence of a wonderful compactification, the toroidal varieties are classified by the toric varieties that dominate the affine 
space. Indeed, $X_{0}$ contains a $T$-subvariety isomorphic to $\mathbb{A}^{\operatorname{rank}(G / H)}$ and there is a morphism $p: X \rightarrow X_{0}$. Finally, the toric variety associated to $X$ is the inverse image of $\mathbb{A}^{\operatorname{rank}(G / H)}$ in $X$.

Theorem C. Let $X$ be a Fano toroidal symmetric variety of rank two with $G$ semisimple and such that the standard compactification is wonderful. Then, up to isomorphism, the fan of the associated toric variety $Z$ has maximal cones

$$
\left\{\operatorname{cone}\left(e_{1}, e_{1}+n e_{2}\right), \operatorname{cone}\left(e_{2}, e_{1}+n e_{2}\right)\right\}
$$

for an appropriate $n \in \mathbb{Z}^{+}$. Here $\left\{e_{1}, e_{2}\right\}$ is the lattice basis associated to $\mathbb{A}^{2} \subset X_{0}$. Moreover $X$ is smooth if and only if $n=1$.

Theorem D. Let $X$ be a quasi-Fano smooth toroidal symmetric variety of rank two with $G$ semisimple and such that the standard compactification is wonderful. Then, up to isomorphism, the fan of the associated toric variety $Z$ has maximal cones

$$
\begin{array}{r}
\text { cone }\left(e_{1}, e_{1}+e_{2}\right), \operatorname{cone}\left(e_{1}+e_{2}, e_{1}+2 e_{2}\right), \ldots, \operatorname{cone}\left(e_{1}+(n-1) e_{2}, e_{1}+n e_{2}\right), \\
\left.\operatorname{cone}\left(e_{1}+n e_{2}, e_{2}\right)\right\}
\end{array}
$$

for an appropriate $n \in \mathbb{Z}^{+}$. Here $\left\{e_{1}, e_{2}\right\}$ is the lattice basis associated to $\mathbb{A}^{2} \subset X_{0}$.

Finally, we classify the smooth Fano symmetric varieties of rank three that are obtainable from a wonderful one by a sequence of blow-ups along $G$-subvarieties (in particular $G$ is semisimple). This class of varieties is quite large; indeed any compact symmetric variety is dominated by a variety obtained from a wonderful variety by a sequence of blow-ups along $G$-subvarieties of codimension two (see [dCoPr85, Theorem 2.4]). In particular, we prove the following necessary condition:

Theorem E. Let $X$ be a Fano symmetric variety of rank three that is obtainable from a wonderful one $X_{0}$ by a sequence of blow-ups along $G$-subvarieties. Then we have the following possibilities:

- $X$ is the wonderful compactification $X_{0}$;

- $X$ is a blow-up of $X_{0}$ along a $G$-subvariety;

- $X$ is obtainable from $X_{0}$ by a blow-up along a G-subvariety, followed by a blowup along a $G$-subvariety of codimension two.

Moreover, there are at most eleven Fano varieties obtainable from a wonderful variety by a sequence of blow-ups. 
This last result on rank-3 varieties can be generalized to varieties obtainable from generic wonderful varieties of rank 3 by a sequence of blow-ups along $G$ subvarieties (without supposing $G / H$ symmetric).

This paper is organized as follows. In $\S \S 1.1-1.5$ we explain the classification of symmetric varieties. Then, in $\S 1.6$ and $\S 1.7$ we define the symmetric varieties which we are most interested in: the wonderful varieties and toroidal varieties. In $\$ 1.8$ we describe the Picard group of a symmetric variety. In $\S 2$ we state and explain our results. In $\S 3$ we prove Theorem A, while in $\S 4$ we prove Theorem B. In $\S 5$ we prove the results in rank three, in particular Theorem E. Finally in $\S 6$ we classify the 2-rank Fano symmetric varieties. This last section is divided into three parts: 1) In $\S 6.1$ we classify the locally factorial (resp. smooth) Fano symmetric varieties with $G$ semisimple; in particular we prove Theorem C. 2) In $\S 6.2$ we classify the locally factorial (resp. smooth) quasi-Fano symmetric varieties with $G$ semisimple; in particular we prove Theorem D. 3) In $\S 6.3$ we classify the locally factorial (resp. smooth) Fano symmetric varieties with $G$ non-semisimple.

\section{$\S 1$. Introduction and notation}

In this section we introduce the necessary notation. The reader interested in the embedding theory of spherical varieties can consult [Kn91], [Br97a] or [T06]. In [Vu90] this theory is explained in the particular case of symmetric varieties.

\section{$\S 1.1$. First definitions}

Let $G$ be a connected reductive algebraic group over $\mathbb{C}$ and let $\theta$ be an involution of $G$. Given a closed subgroup $H$ such that $G^{\theta} \subset H \subset N_{G}\left(G^{\theta}\right)$, we say that $G / H$ is a symmetric space and that $H$ is a symmetric subgroup. A normal $G$-variety is called a spherical variety if it contains a dense $B$-orbit ( $B$ is a chosen Borel subgroup of $G$ ). We say that a subtorus of $G$ is split if $\theta(t)=t^{-1}$ for all its elements $t$; moreover it is a maximal split torus if it has maximal dimension. A maximal torus containing a maximal split torus is maximally split; any maximally split torus is $\theta$ stable (see [T06, Lemma 26.5]). We fix arbitrarily a maximal split torus $T^{1}$ and a maximally split torus $T$ containing $T^{1}$. We denote by $n$ the rank of $G$ (i.e. the dimension of $T$ ). We can choose a Borel subgroup $B \supset T$ such that, for any positive root $\alpha$, either $\theta(\alpha)=\alpha$ or $\theta(\alpha)$ is negative. Moreover, $B H$ is dense in $G$ (see [dCoPr83, Lemma 1.2 and Proposition 1.3]). In particular, every normal equivariant open embedding of $G / H$ is spherical; we call it a symmetric variety. Let $R_{G}$ be the root system of $G$ with respect to $T$ and let $R_{G}^{+}$be the set of positive roots with respect to $B$.

We can assume that $G$ is the direct product of a simply connected, semisimple group with a central split torus (see [Vu90, §2.1] or [Ru07, Remark 1]). 


\section{$\S 1.2$. Colored fans}

Now, we introduce some details about the classification of the symmetric varieties with fixed $G / H$ by their colored fans (this classification is defined more generally for spherical varieties). Let $D(G / H)$ be the set of $B$-stable prime divisors of $G / H$; its elements are called colors. We say that a spherical variety is simple if it contains only one closed orbit. Let $X$ be a simple symmetric variety with closed orbit $Y$. We define the set of colors of $X$ as the subset $D(X)$ of $D(G / H)$ consisting of the colors whose closure in $X$ contains $Y$. To each prime divisor $D$ of $X$, we can associate the normalized discrete valuation $v_{D}$ of $\mathbb{C}(G / H)$ whose ring is $\mathcal{O}_{X, D} ; D$ is $G$-stable if and only if $v_{D}$ is $G$-invariant. Let $N$ be the set of all $G$-invariant valuations of $\mathbb{C}(G / H)$ taking values in $\mathbb{Z}$ and let $N(X)$ be the set of valuations associated to the $G$-stable prime divisors of $X$. Observe that each irreducible component of $X \backslash(G / H)$ has codimension one, because $G / H$ is affine. Let $S:=$ $T / T \cap H \simeq T \cdot(e H / H)$. One can show that the group $\mathbb{C}(G / H)^{(B)} / \mathbb{C}^{*}$ is isomorphic to the character group $\chi(S)$ of $S$ (see [Vu90, $\S 2.3]$ ); in particular, it is a free abelian group. We define the rank of $G / H$ as the rank of $\chi(S)$. We can identify the dual group $\operatorname{Hom}_{\mathbb{Z}}\left(\mathbb{C}(G / H)^{(B)} / \mathbb{C}^{*}, \mathbb{Z}\right)$ with the group $\chi_{*}(S)$ of one-parameter subgroups of $S$; so we can identify $\chi_{*}(S)_{\mathbb{R}}$ with $\operatorname{Hom}_{\mathbb{Z}}(\chi(S), \mathbb{R})$. The restriction map to $\mathbb{C}(G / H)^{(B)} / \mathbb{C}^{*}$ is injective over $N$ (see [Br97a, $\S 3.1$, Corollaire 3]), so we can identify $N$ with a subset of $\chi_{*}(S)_{\mathbb{R}}$. We say that $N$ is the valuation monoid of $G / H$. For each color $D$, we define $\rho(D)$ as the restriction of $v_{D}$ to $\chi(S)$. In general, the map $\rho: D(G / H) \rightarrow \chi_{*}(S)_{\mathbb{R}}$ is not injective. We will describe more explicitly $\rho(D(G / H))$ and $N$ in $\S 1.5$. Let $C(X)$ be the cone in $\chi_{*}(S)_{\mathbb{R}}$ generated by $N(X)$ and $\rho(D(X))$. We say that the pair $(C(X), D(X))$ is the colored cone of $X$; it determines $X$ uniquely (see [Br97a, $\S 3.3$, Théorème]).

Let $Y$ be an orbit of a symmetric variety $X$. The set $\{x \in X: \overline{G \cdot x} \supset Y\}$ is an open simple $G$-subvariety of $X$ with closed orbit $Y$, because any spherical variety contains finitely many $G$-orbits. Let $\left\{X_{i}\right\}$ be the set of open simple subvarieties of $X$ and define the set of colors of $X, D(X)$, as $\bigcup_{i \in I} D\left(X_{i}\right)$. The family $\mathcal{F}(X):=$ $\left\{\left(C\left(X_{i}\right), D\left(X_{i}\right)\right)\right\}_{i \in I}$ is called the colored fan of $X$ and determines $X$ completely (see [Br97a, §3.4, Théorème 1]). Moreover $X$ is compact if and only if cone $(N)$ is contained in the support $|\mathcal{F}(X)|:=\bigcup_{i \in I} C\left(X_{i}\right)$ of the colored fan (see [Br97a, $\S 3.4$, Théorème 2]).

Let $(C, F)$ be a colored cone of $X$. We say that the blow-up of $X$ along the subvariety associated to $(C, F)$ is the blow-up of $X$ along $(C, F)$. Moreover, we say that blow-up of a variety along a subvariety of codimension $i$ is an $i$-blow-up.

Given a symmetric variety $X$ we denote by $\Delta$ (or $\Delta_{X}$ ) the fan associated to the colored fan of $X$, by $\Delta(i)$ the set of $i$-dimensional cones in $\Delta$ and by 
$\Delta[p]$ the set of primitive generators of 1-dimensional cones of $\Delta$. The fan $\Delta$ is formed by all the faces of the cones $C$ such that there is a colored cone $(C, F) \in$ $\mathcal{F}(X)$. The toric varieties are a special case of symmetric varieties. If $X$ is a toric variety, then $D(G / H)$ is empty and we need only consider the fan $\Delta_{X}$ (actually the theory of colored fans is a generalization of the classification of toric varieties by fans).

\section{$\S 1.3$. Restricted root system}

To describe the sets $N$ and $\rho(D(G / H))$, we associate a root system to $G / H$. We can identify $\chi\left(T^{1}\right)_{\mathbb{R}}$ with $\chi(S)_{\mathbb{R}}$ because $\left[\chi(S): \chi\left(T^{1}\right)\right]$ is finite. We denote again by $\theta$ the involution induced on $\chi(T)_{\mathbb{R}}$. The inclusion $T^{1} \subset T$ induces an isomorphism of $\chi\left(T^{1}\right)_{\mathbb{R}}$ with the $(-1)$-eigenspace of $\chi(T)_{\mathbb{R}}$ under the action of $\theta$ (see [T06, §26]). Denote by $W_{G}$ the Weyl group of $G$ (with respect to $T$ ). We can identify $\chi\left(T^{1}\right)_{\mathbb{R}}$ with its dual $\chi_{*}\left(T^{1}\right)_{\mathbb{R}}$ by the restriction $(\cdot, \cdot)$ to $\chi\left(T^{1}\right)_{\mathbb{R}}$ of a fixed $W_{G}$-invariant non-degenerate symmetric bilinear form on $\chi(T)_{\mathbb{R}}$. Let $R_{G}^{0}$ be the set of roots fixed by $\theta$ and let $R_{G}^{1}=R_{G} \backslash R_{G}^{0}$ and $R_{G}^{i,+}:=R_{G}^{i} \cap R_{G}^{+}$.

The set $R_{G, \theta}:=\left\{\beta-\theta(\beta): \beta \in R_{G}^{1}\right\}$ is a (possibly non-reduced) root system in $\chi(S)_{\mathbb{R}}$ (see [Vu90, $§ 2.3$ Lemme]), which we call the restricted root system of $(G, \theta)$; we call the non-zero $\beta-\theta(\beta)$ the restricted roots. If $G$ is semisimple, then the rank of $R_{G, \theta}$ is equal to the rank of $G / H$. Usually we denote by $\beta$ (resp. by $\alpha$ ) a root of $R_{G}$ (resp. of $R_{G, \theta}$ ); often we denote by $\varpi$ (resp. by $\omega$ ) a weight of $R_{G}$ (resp. of $R_{G, \theta}$ ). We denote by $\bar{R}_{G}=\left\{\beta_{1}, \ldots, \beta_{n}\right\}$ the basis of $R_{G}$ associated to $B$ and by $\varpi_{1}, \ldots, \varpi_{n}$ the fundamental weights of $R_{G}$. Let $\bar{R}_{G}^{i}$ be $\bar{R}_{G} \cap R_{G}^{i}$. We denote by $\alpha_{1}, \ldots, \alpha_{s}$ the elements of the basis $\bar{R}_{G, \theta}:=\left\{\beta-\theta(\beta): \beta \in \bar{R}_{G}^{1}\right\}$ of $R_{G, \theta}$. If $R_{G, \theta}$ is irreducible we order $\bar{R}_{G, \theta}$ as in [Bo68]. Let $b_{i}$ be equal to $\frac{1}{2}$ if $2 \alpha_{i}$ belongs to $R_{G, \theta}$ and equal to one otherwise; for each $i$ we define $\alpha_{i}^{\vee}$ as the coroot $\frac{2 b_{i}}{\left(\alpha_{i}, \alpha_{i}\right)} \alpha_{i}$. The set $\left\{\alpha_{1}^{\vee}, \ldots, \alpha_{s}^{\vee}\right\}$ is a basis of the dual root system $R_{G, \theta}^{\vee}$. We call the elements of $R_{G, \theta}^{\vee}$ the restricted coroots. Let $\omega_{1}, \ldots, \omega_{s}$ be the fundamental weights of $R_{G, \theta}$ with respect to $\left\{\alpha_{1}, \ldots, \alpha_{s}\right\}$ and let $\omega_{1}^{\vee}, \ldots, \omega_{s}^{\vee}$ be the fundamental weights of $R_{G, \theta}^{\vee}$ with respect to $\left\{\alpha_{1}^{\vee}, \ldots, \alpha_{s}^{\vee}\right\}$. Let $C^{+}$be the positive closed Weyl chamber of $\chi(S)_{\mathbb{R}}$ and let $C^{-}:=-C^{+}$.

There is a permutation $\bar{\theta}$ of $\bar{R}_{G}^{1}$ such that, for all $\beta \in \bar{R}_{G}^{1}, \theta(\beta)+\bar{\theta}(\beta)$ is a linear combination of roots in $\bar{R}_{G}^{0}$. We say that a dominant weight $\varpi \in \chi(T)$ is a spherical weight if $V(\varpi)$ contains a non-zero vector fixed by $G^{\theta}$. In this case, $V(\varpi)^{G^{\theta}}$ is one-dimensional and $\theta(\varpi)=-\varpi$, so $\varpi$ belongs to $\chi(S)_{\mathbb{R}}$. One can show that the set of dominant weights of $R_{G, \theta}$ is the set of spherical weights and that $C^{+}$is the intersection of $\chi(S)_{\mathbb{R}}$ with the positive closed Weyl chamber of $R_{G}$. Suppose $\beta_{j}-\theta\left(\beta_{j}\right)=\alpha_{i}$. Then $\omega_{i}$ is a positive multiple of $\varpi_{j}+\varpi_{\bar{\theta}(j)}$. 
More precisely, we have the following possibilities: (i) $\omega_{i}=\varpi_{j}+\varpi_{\bar{\theta}(j)}$ if $\bar{\theta}(j) \neq j$; (ii) $\omega_{i}=2 \varpi_{j}$ if $\bar{\theta}(j)=j$ and $\beta_{j}$ is orthogonal to $R_{G}^{0}$; (iii) $\omega_{i}=\varpi_{j}$ in the other cases (see [ChMa03, Theorem 2.3] or [T06, Proposition 26.4]). We say that a spherical weight is regular if it is strictly dominant as weight of the restricted root system.

\section{§1.4. Classification of symmetric spaces}

We say that $(G, \theta)$ is indecomposable if the unique normal, connected, $\theta$-stable subgroup of $G$ is the trivial one. Otherwise, we say that $(G, \theta)$ is decomposable. In this case $G / H$ is, up to a finite cover, a product of smaller symmetric spaces. Indeed $G=G_{1} \times G_{2}$ where $G_{1}$ and $G_{2}$ are $\theta$-stable, normal subgroups, so $G / G^{\theta}=G_{1} / G_{1}^{\theta} \times G_{2} / G_{2}^{\theta}$. If $\theta$ is indecomposable, there are three possibilities: (i) $G$ is simple; (ii) $G=\dot{G} \times \dot{G}$ with $\dot{G}$ simple and $\theta(x, y)=(y, x)$; (iii) $G=\mathbb{C}^{*}$ and $\theta(t)=t^{-1}$. The classification, up to conjugation, of the involutions of a simple group is as in Tables 1 and 2 (see also [Wa72, §1.1] and [T06, Table 5.9]). In the fourth column of these tables the Satake diagram of the involution is shown. The Satake diagram of any involution $(G, \theta)$ is obtained from the Dynkin diagram of $G$ as follows: 1) the vertices corresponding to elements of $\bar{R}_{G}^{0}$ (resp. of $\bar{R}_{G}^{1}$ ) are black (resp. white); 2) two simple roots $\beta_{1}, \beta_{2} \in \bar{R}_{G}^{1}$ such that $\bar{\theta}\left(\beta_{1}\right)=\beta_{2}$ are linked by a double-headed arrow. Finally, if $G=\dot{G} \times \dot{G}$ (with $\dot{G}$ simple) and $\theta(x, y):=(y, x)$, then $R_{G}^{0}$ is empty and the Satake diagram consists of two copies of the Dynkin diagram of $\dot{G}$. Moreover, each vertex of a copy of the Dynkin diagram of $\dot{G}$ is joined to the corresponding vertex of the other copy. For example, if $\dot{G}$ is $S L_{l+1}$, we have the following diagram:

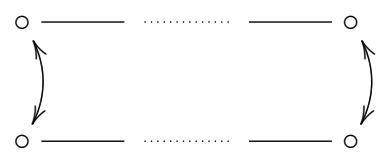

In $\S 4$, we will need to write the simple restricted roots as a linear combination of simple roots of $G$ for the following involutions (see also [T06, Table 5.9]): 1) The involution $\theta$ of type $A I I$; in this case $G / G^{\theta}=S L_{2 n} / S p_{2 n}$. Let $I_{n} \in M_{n}(\mathbb{C})$ be the identity matrix and let $\Omega_{n}$ be $\left(\begin{array}{cc}0 & I_{n} \\ -I_{n} & 0\end{array}\right)$; then $\theta(g)=\Omega_{n}\left(g^{t}\right)^{-1} \Omega_{n}^{-1}$ for all $g \in S L_{2 n}$. 2) The involution $\theta$ of type $C I I$; in this case $G / G^{\theta}=S p_{2 n} /\left(S p_{2 l} \times S p_{2 n-2 l}\right)$. Let $K_{n, l}$ be the diagonal matrix with entries $\left(I_{l},-I_{n-l}, I_{l},-I_{n-l}\right)$; then $\theta(g)=$ $K_{n, l} g K_{n, l}$ for all $g \in S p_{2 n}$. 3) The involution $\theta$ of type $D I I I$ with $n=2 l+1$; then $G / G^{\theta}=S L_{4 l+2} / G L_{2 l+1}$ and $\theta(g)=\Omega_{n} g \Omega_{n}^{-1}$ for all $g \in S L_{4 l+2}$. These descriptions allow one to prove that $\alpha_{i}=\beta_{i-1}+2 \beta_{i}+\beta_{i+1}$ for each $i<l$. 
Table 1. Involutions of simple groups

\begin{tabular}{|c|c|c|c|c|c|}
\hline type & $G$ & $G^{\vartheta}$ & Satake diagram & $R_{G, \theta}$ & $\operatorname{rank} G / G^{\theta}$ \\
\hline$A I$ & $S L_{l+1}$ & $S O_{l+1}$ & $0-\cdots-0$ & $A_{l}$ & $l \geq 1$ \\
\hline$A I I$ & $S L_{2 l+2}$ & $S p_{2 l+2}$ & $\bullet-$ & $A_{l}$ & $l \geq 1$ \\
\hline$A I I I$ & $S L_{n+1}$ & $S\left(G L_{l} \times G L_{n+1-l}\right)$ & & $B C_{l}$ & $\frac{n}{2} \geq l \geq 2$ \\
\hline$A I I I$ & $S L_{2 l}$ & $S\left(G L_{l} \times G L_{l}\right)$ & 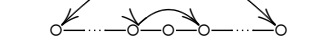 & $C_{l}$ & $l \geq 2$ \\
\hline$A I V$ & $S L_{n+1}$ & $G L_{n}$ & $0 \leqslant$ & $B C_{1}$ & $n>l=1$ \\
\hline$B I$ & $S O_{2 n+1}$ & $S\left(O_{l} \times O_{2 n+1-l}\right)$ & $0-\cdots-c$ & $B_{l}$ & $n \geq l \geq 2$ \\
\hline$B I I$ & $S O_{2 n+1}$ & $S\left(O_{1} \times O_{2 n}\right)$ & o- & $A_{1}$ & $n-1 \geq l=1$ \\
\hline$C I$ & $S p_{2 l}$ & $G L_{l}$ & o-.. & $C_{l}$ & $l \geq 3$ \\
\hline$C I I$ & $S p_{2 n}$ & $S p_{2 l} \times S p_{2 n-2 l}$ & $\bullet-0-\bullet-$ & $B C_{l}$ & $\frac{n-1}{2} \geq l \geq 1$ \\
\hline$C I I$ & $S p_{4 l}$ & $S p_{2 l} \times S p_{2 l}$ & $\bullet-0-$ & $C_{l}$ & $l \geq 2$ \\
\hline$D I$ & $S O_{2 n}$ & $S\left(O_{l} \times O_{2 n-l}\right)$ & & $B_{l}$ & $n-2 \geq l \geq 2$ \\
\hline$D I$ & $S O_{2 l+2}$ & $S\left(O_{l} \times O_{l+2}\right)$ & & $B_{l}$ & $l \geq 3$ \\
\hline$D I$ & $\mathrm{SO}_{2 l}$ & $S\left(O_{l} \times O_{l}\right)$ & & $D_{l}$ & $l \geq 4$ \\
\hline$D I I$ & $S_{2 n}$ & $S\left(O_{1} \times O_{2 n-1}\right)$ & & $A_{1}$ & $l=1$ \\
\hline$D I I I$ & $\mathrm{SO}_{4 l}$ & $G L_{2 l}$ & & $C_{l}$ & $l \geq 2$ \\
\hline$D I I I$ & $\mathrm{SO}_{4 l+2}$ & $G L_{2 l+1}$ & & $B C_{l}$ & $l \geq 2$ \\
\hline$E I$ & $E_{6}$ & $C_{4}$ & O-O-O-O-O & $E_{6}$ & $l=6$ \\
\hline$E I I$ & $E_{6}$ & $A_{5} \times A_{1}$ & $\begin{array}{c}0-0-0-0-0 \\
1 \\
0\end{array}$ & $F_{4}$ & $l=4$ \\
\hline EIII & $E_{6}$ & $D_{5} \times \mathbb{C}^{*}$ & $0 \leq-\bullet$ & $B C_{2}$ & $l=2$ \\
\hline$E I V$ & $E_{6}$ & $F_{4}$ & 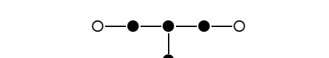 & $A_{2}$ & $l=2$ \\
\hline$E V$ & $E_{7}$ & $A_{7}$ & ০-০-o- & $E_{7}$ & $l=7$ \\
\hline$E V I$ & $E_{7}$ & $D_{6} \times A_{1}$ & $\bullet-0-\bullet-0-0-0$ & $F_{4}$ & $l=4$ \\
\hline$E V I I$ & $E_{7}$ & $E_{6} \times \mathbb{C}^{*}$ & O-O- $\bullet-\bullet-\circ$ & $C_{3}$ & $l=3$ \\
\hline
\end{tabular}




\begin{tabular}{|c|c|c|c|c|c|}
\hline EVIII & $E_{8}$ & $D_{8}$ & 每 & $E_{8}$ & $l=8$ \\
\hline$E I X$ & $E_{8}$ & $E_{7} \times A_{1}$ & 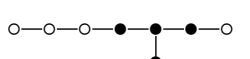 & $F_{4}$ & $l=4$ \\
\hline$F I$ & $F_{4}$ & $C_{3} \times A_{1}$ & $0-0 \Rightarrow 0-0$ & $F_{4}$ & $l=4$ \\
\hline FII & $F_{4}$ & $B_{4}$ & $\bullet-\bullet \Rightarrow \bullet-\circ$ & $B C_{1}$ & $l=1$ \\
\hline G & $G_{2}$ & $A_{1} \times A_{1}$ & $\circ \equiv \circ$ & $G_{2}$ & $l=2$ \\
\hline
\end{tabular}

\section{$\S 1.5$. The sets $N$ and $D(G / H)$}

The set $N$ equals $C^{-} \cap \chi_{*}(S)$; in particular, it consists of the lattice vectors of the rational, polyhedral, convex cone $C^{-}=\operatorname{cone}(N)$. The set $\rho(D(G / H))$ equals $\bar{R}_{G, \theta}^{\vee}$ and any fiber $\rho^{-1}\left(\alpha^{\vee}\right)$ contains at most two colors.

Now, we explain the following property: if $\rho$ is injective, then the symmetric varieties are classified by the fan associated to the colored fan. For any simple spherical variety $X, N(X)$ is formed by the primitive generators of the 1-faces of $C(X)$ which are contained in cone $(N)$. When $X$ is symmetric, also $\rho(X)$ can be recovered from $C(X)$ : its elements generate the 1-faces of $C(X)$ which are not contained in $C^{-}$. Indeed, given cone $\left(\alpha_{1}^{\vee}, \ldots, \alpha_{r}^{\vee}, \omega_{1}^{\vee}, \ldots, \omega_{s}^{\vee}\right)$ with $\omega_{1}^{\vee}, \ldots, \omega_{s}^{\vee}$ antidominant, $\alpha_{j}^{\vee}$ is the unique generator of a cone such that $\left(\alpha_{j}^{\vee}, \alpha_{j}\right)>0$.

Finally, we state some technical properties of $D(G / H)$, which we will use to study the local factoriality and the smoothness of a symmetric variety. If $(G, \theta)$ is indecomposable, then the number of colors is at most $\operatorname{rank}(G / H)+1$. If moreover $\sharp D(G / H)>\operatorname{rank}(G / H)$, we say that $G / H$ is Hermitian; we have two possibilities (see [Ru07, pp. 7-8] or [T06, pp. 177-178]): 1) $\left.G^{\theta}=H=N_{G}\left(G^{\theta}\right) ; 2\right) H=G^{\theta}$ and $\left[G^{\theta}: N_{G}\left(G^{\theta}\right)\right]=2$. In the last case any element of $N_{G}\left(G^{\theta}\right) \backslash G^{\theta}$ exchanges two colors and $R_{G, \theta}$ has type $A_{1}, B_{2}$ or $C_{n}$. We say that a simple restricted root $\alpha$ is exceptional if $\sharp \rho^{-1}\left(\alpha^{\vee}\right)=2$ and $2 \alpha$ is a restricted root. In this case the irreducible factor of $R_{G, \theta}$ containing $\alpha$ is associated to an indecomposable factor of $G / G^{\theta}$ as in 1$)$. We then say that also $(G, \theta)$ and any symmetric variety (with open orbit $G / H)$ are exceptional. We denote by $D_{\alpha}$ the sum of the colors in $\rho^{-1}\left(\alpha^{\vee}\right)$ and by $D_{\omega}$ the $G$-stable divisor corresponding to $\left(\mathbb{R}^{\geq 0} \omega, \emptyset\right) \in \mathbb{F}(X)$.

\section{§1.6. Wonderful symmetric varieties}

In this section we define the wonderful compactification of $G / H$, while in the following section we will use this variety to describe the toroidal symmetric varieties. A wonderful compactification exists only if $N(H) / H$ is finite; in particular the connected center of $G$ acts trivially on $G / H$. Thus we can suppose that $G$ is semisimple. In this case one can define a canonical compactification which has many properties. This compactification, called the standard compactification $X_{0}$ 
(or the standard symmetric variety), is associated to ( $\operatorname{cone}(N), \emptyset)$ and it is the maximal simple compactification of $G / H$ in the dominant order. We define $e_{i}$ as the primitive positive multiple of $-\omega_{i}^{\vee}$ (in $\chi_{*}(S)$ ), so $\Delta_{X_{0}}[p]=\left\{e_{1}, \ldots, e_{l}\right\}$.

The standard compactification is called wonderful if it is also smooth. A standard symmetric variety is wonderful if and only if $\chi_{*}(S)=\bigoplus \mathbb{Z} e_{i}$. De Concini and Procesi have proved that $X_{0}$ is wonderful if $H=N_{G}\left(G^{\theta}\right)$, or equivalently $\chi_{*}(S)=\bigoplus \mathbb{Z} \omega_{i}^{\vee}$ (see [dCoPr83, Theorem 3.1]). Looking at the Cartan matrix of $R_{G, \theta}$ it is not difficult to prove that a standard indecomposable symmetric variety $X$ is wonderful exactly in the following two cases: (i) $H=N_{G}\left(G^{\theta}\right)$; (ii) $R_{G, \theta}$ has type $A_{1}, B_{2}$ or $C_{l}$.

A standard (resp. wonderful) symmetric variety is the standard (resp. wonderful) compactification of a symmetric space.

\section{$\S 1.7$. Toroidal symmetric varieties}

In this section we want to define a special class of varieties, which are closely related to toric varieties. We say that a spherical variety is toroidal if $D(X)=\emptyset$. We want to explain how to associate a toric variety $Z$ to a toroidal symmetric variety $X$. Many local properties of $X$ can be studied on $Z$. Moreover, in the toroidal case the key step of our classification is the study of the anticanonical bundle of the associated toric varieties. In the rest of this section we suppose that $G$ is semisimple.

The standard compactification $X_{0}$ contains an affine toric $S$-variety $Z_{0}$, which is a quotient of an affine space by a finite group; moreover $\operatorname{dim} Z_{0}=\operatorname{rank} X_{0}$. The toroidal varieties are the symmetric varieties which dominate the standard compactification and are in one-to-one correspondence with the $S$-toric varieties which dominate $Z_{0}$ (see $[\mathrm{dCoPr} 83, \S 2.3]$ and $[\mathrm{dCoPr} 85, \S 5.2]$ ).

Let $P$ be the stabilizer of the $B$-stable affine open set $U:=X_{0} \backslash \bigcup_{D \in D(G / H)} \bar{D}$. This open set is $P$-isomorphic to $R_{u} P \times Z_{0}$, where $R_{u} P=\prod_{\beta \in R_{G}^{1+}+U_{\beta}}$ is the unipotent radical of $P$. To any toroidal variety $X$ we associate the inverse image $Z$ of $Z_{0}$ under the projection $X \rightarrow X_{0}$. Moreover, $X \backslash \bigcup_{D \in D(G / H)} \bar{D}$ is $P$-isomorphic to $R_{u} P \times Z$. Thus the restriction of the anticanonical bundle of $X$ to $Z$ is isomorphic to the anticanonical bundle of $Z$. In particular, if $X$ is Fano then $-K_{Z}$ is ample. Note that $Z_{0}$ is an affine space if and only if $X_{0}$ is wonderful.

\section{$\S 1.8$. The Picard group}

The class group of a symmetric variety is generated by the classes of the $B$ stable prime divisors modulo the relations $\operatorname{div}(f)$ with $f \in \mathbb{C}(G / H)^{(B)}$. Indeed $\mathrm{Cl}(B H / H)=\operatorname{Pic}(B H / H)$ is trivial. Given $\omega \in \chi(S)$ we denote by $f_{\omega}$ the element of $\mathbb{C}(G / H)^{(B)}$ with weight $\omega$ and such that $f_{\omega}(H / H)=1$. 
A Weyl divisor $\sum_{D \in D(G / H)} a_{D} D+\sum_{E \in N(X)} b_{E} E$ is a Cartier divisor if and only if, for any $(C, F) \in \mathcal{F}(X)$, there is $h_{C} \in \chi(S)$ such that $h_{C}(E)=a_{E}$ for all $E \in C$ and $h_{C}(\rho(D))=a_{D}$ for all $D \in F$. Let $P L^{\prime}(X)$ be the set of functions on the support $|\mathcal{F}(X)|$ that are: 1) linear on each colored cone; 2) integer on $\chi_{*}(S) \cap|\mathcal{F}(X)|$. Let $L(X) \subset P L^{\prime}(X)$ be the subset composed of the restrictions of linear functions and let $P L(X):=P L^{\prime}(X) / L(X)$. Then $\left\{h_{C}\right\}$, corresponding to any Cartier divisor, defines an element of $P L(X)$. If $X$ is compact, there is an exact sequence (see [Br89, Théorème 3.1])

$$
0 \rightarrow \bigoplus_{D \in D(G / H) \backslash D(X)} \mathbb{Z} D \rightarrow \operatorname{Pic}(X) \rightarrow P L(X) \rightarrow 0 .
$$

A Cartier divisor is globally generated (resp. ample) if and only if the associated function is convex (resp. strictly convex) and $h_{C}(\rho(D)) \leq a_{D}$ (resp. $\left.h_{C}(\rho(D))<a_{D}\right)$ for all $(C, F) \in \mathcal{F}(X)$ and for all $D \in D(G / H) \backslash F$. Thus, a Cartier divisor on a projective symmetric variety is nef if and only if it is globally generated. Given any linearized line bundle $L$, the space $H^{0}(X, L)$ is a multiplicity free $G$-module and, if $L$ is globally generated, the highest weights of $H^{0}(X, L)$ are the elements of $\chi(S) \cap \operatorname{hull}\left(\left\{h_{C}\right\}_{\operatorname{dim} C=l}\right)$, where $l=\operatorname{rank}(G / H)$ and $\operatorname{hull}\left(\left\{x_{1}, \ldots, x_{m}\right\}\right)$ is the convex hull of $x_{1}, \ldots, x_{m}$ (see $\left.[\operatorname{Br} 89, \S 3]\right)$. Moreover, a nef $G$-stable Cartier divisor on a projective symmetric variety is big if and only if the associated piecewise linear function $h$ is such that $\left(\sum_{C \in \Delta(l)} h_{C}, R^{\vee}\right) \neq 0$ for each irreducible factor $R^{\vee}$ of $R_{G, \theta}^{\vee}$ (see [Ru09, Theorem 4.2]). In particular, when $\theta$ is indecomposable every non-zero nef $G$-stable divisor is big.

When $X$ is toroidal we have an exact split sequence

$$
0 \rightarrow \operatorname{Pic}\left(X_{0}\right) \rightarrow \operatorname{Pic}(X) \rightarrow \operatorname{Pic}(Z) \rightarrow 0
$$

A normal variety $X$ is locally factorial if the Picard group is isomorphic to the class group, while $X$ is $\mathbb{Q}$-factorial if $\operatorname{Pic}(X)_{\mathbb{Q}} \cong \mathrm{Cl}(X)_{\mathbb{Q}}$. A simple symmetric variety associated to a colored cone $(C, F)$ is locally factorial if: (i) $C$ is generated by a subset of a basis of $\chi_{*}(S)$ and (ii) $\rho$ is injective over $F$ (see [Br97b] for a general statement in the spherical case). When the variety is toroidal, local factoriality is equivalent to smoothness.

We can describe an anticanonical divisor by [Br97b, Theorem 4.2] and by the local description of $X_{0}$; we also use the fact that, by the cited theorem, the non- $G$ stable part of such a divisor depends only on the open $G$-orbit. An anticanonical divisor $-K_{X}$ of $X$ is $\sum_{\alpha \in \bar{R}_{G, \theta}^{\vee}} a_{\alpha} D_{\alpha}+\sum_{E \in N(X)} E$ with $\sum a_{\alpha} \omega_{\alpha}=2 \delta-2 \delta_{0}$. Here $2 \delta:=2 \delta_{R_{G}}=\sum_{\alpha \in R_{G}^{+}} \omega_{\alpha}$ is the sum of all the positive roots of $R_{G}$, while $2 \delta_{0}:=2 \delta_{R_{G}^{0}}$ is the sum of the positive roots in $R_{G}^{0}$. 
Let $k\left(\right.$ or $k_{X}$ ) be the piecewise linear function associated to $-K_{X}$. The anticanonical divisor $-K_{X}$ is linearly equivalent to a unique $G$-stable divisor $-\widetilde{K}_{X}$. The piecewise linear function $\widetilde{k}$ ( or $\widetilde{k}_{X}$ ) associated to $-\widetilde{K}_{X}$ equals $k-2 \delta+2 \delta_{0}$ over $N(X)$ and to 0 over $\rho(D(X))$. Indeed $-\widetilde{K}_{X}$ is $-K_{X}+\operatorname{div}\left(\prod_{\alpha \in \bar{R}_{G, \theta}} f_{\alpha}^{a_{\alpha}}\right)$, where $f_{\alpha} \in \mathbb{C}(G / H)^{(B)}$ is an equation of $D_{\alpha}$ (of weight $\omega_{\alpha}$ ). In particular $\widetilde{k}=k-2 \delta+2 \delta_{0}$ if $X$ is toroidal.

\section{\$2. Statement of the main theorems}

In this section we state the main theorems of this work. In the following, unless explicitly stated, we always suppose that $G$ is semisimple (we will consider the general reductive case mainly in $\$ 2.4 .3$ and $\$ 6.3)$. Moreover, we often denote the normalizer $N_{G}(H)$ by $N(H)$.

\section{§2.1. Fano standard varieties}

When the rank of $G / H$ is one, the standard compactification $X_{0}$ of $G / H$ is the unique $G$-equivariant compactification. In that case $X_{0}$ is a homogeneous projective variety with respect to $\operatorname{Aut}^{0}(X)$ by [A83]; moreover it is wonderful and Fano, because either it is $\mathbb{P}^{n} \times \mathbb{P}^{n}$ or it has Picard number one. A standard symmetric variety is always $\mathbb{Q}$-factorial; in particular, $K_{X}$ is a $\mathbb{Q}$-Cartier divisor. We prove that in general a standard symmetric variety is a quasi- $\mathbb{Q}$-Fano variety; moreover we classify the Fano ones.

First of all, we reduce ourselves to the indecomposable case. Write $(G, \theta)$ as a product $\prod\left(G_{j}, \theta\right)$ of indecomposable involutions and let $X_{j}$ be a standard compactification of $G_{j} /\left(G_{j} \cap H\right)$. If $X$ is wonderful then also the $X_{j}$ are wonderful and $X=\prod X_{j}$ (see [Ru09, Corollary 2.1]).

Lemma 2.1. The variety $X$ is (quasi-) $\mathbb{Q}$-Fano if and only if all the $X_{j}$ are (quasi-) $\mathbb{Q}$-Fano.

Proof. The weight $\widetilde{k}_{X}$ equals $\sum \widetilde{k}_{X_{j}}$.

In $\S 6.3$ we will prove the following result, which in particular implies Theorem A.

Theorem 2.1. Let $X$ be a standard indecomposable symmetric variety. Let $n$ be the rank of $G$ and let $l$ be the rank of $X$. Then:

- The anticanonical divisor of $X$ is always a nef and big $\mathbb{Q}$-divisor.

- Suppose $X$ is wonderful. Then it is not a Fano variety if and only if the following three properties hold: (i) if the involution induced on $\chi(S)_{\mathbb{R}}$ is -id; (ii) $R_{G, \theta}$ is different from $A_{l}$ and $B_{l}$; (iii) $H=N_{G}\left(G^{\theta}\right)$. 
Table 2. Non- $\mathbb{Q}$-Fano standard indecomposable symmetric varieties

\begin{tabular}{cccc}
\hline$G / H$ & $\theta$ & $n, l$ & wonderful \\
\hline Spin $_{2 n+1} /\left(\operatorname{Spin}_{l} \times \operatorname{Spin}_{2 n+1-l}\right)$ & $B I$ & $n \geq l \geq 4$ & no \\
$\operatorname{Spin}_{7} /\left(\operatorname{Spin}_{3} \times \operatorname{Spin}_{4}\right)$ & $B I$ & $n=l=3$ & no \\
$\operatorname{Sp}_{2 n} / N\left(G L_{n}\right)$ & $C I$ & $n=l \geq 3$ & yes \\
$\operatorname{Spin}_{2 l} / N\left(\operatorname{Spin}_{l} \times \operatorname{Spin}_{l}\right)$ & $D I$ & $n=l \geq 4$ & yes \\
$\operatorname{Spin}_{2 n} / H$ & $D I$ & $n=l \geq 6$ & no \\
$\left(G^{\theta} \subset H \subsetneq N\left(G^{\theta}\right)\right)$ & $D I$ & $n>l \geq 4$ & no \\
$\operatorname{Spin}_{2 n} /\left(\operatorname{Spin}_{l} \times \operatorname{Spin}_{2 n-l}\right)$ & $D I$ & $n=4, l=3$ & no \\
$\operatorname{Spin}_{8} /\left(\operatorname{Spin}_{3} \times \operatorname{Spin}_{5}\right)$ & $E I$ & $n=l=6$ & yes \\
$E_{6} / N\left(C_{4}\right)$ & $E V$ & $n=l=7$ & yes \\
$E_{7} / N\left(A_{7}\right)$ & $E V$ & $n=l=7$ & no \\
$E_{7} / A_{7}$ & $E V I I I$ & $n=l=8$ & yes \\
$E_{8} / D_{8}$ & $F I$ & $n=l=4$ & yes \\
$F_{4} /\left(C_{3} \times A_{1}\right)$ & $G$ & $n=l=2$ & yes \\
$G_{2} /\left(A_{1} \times A_{1}\right)$ & & &
\end{tabular}

- The standard indecomposable varieties whose anticanonical divisor is not ample are compactifications of the symmetric spaces in Table 2.

\section{$\S 2.2$. Fano blow-ups of a wonderful symmetric variety along closed orbits}

In this section we state a partial result in arbitrary rank. We restrict ourselves to the smooth toroidal case. In $\S 1.7$ we have associated a toric variety $Z$ to any toroidal symmetric variety $X$; moreover $-K_{Z}$ is ample if $X$ is Fano. For toric varieties of rank 2 one can easily prove the following property:

(*) Let $Z$ be a smooth toric variety of rank 2 and let $Z^{\prime}$ be a smooth toric variety birationally proper over $Z$. If the anticanonical bundle of $Z^{\prime}$ is ample, then also the anticanonical bundle of $Z$ is ample.

This allows one to prove easily that a smooth toric variety proper over $\mathbb{A}^{2}$ with ample anticanonical bundle is either $\mathbb{A}^{2}$ or its blow-up at the origin. We would use a property like $(*)$ to classify the smooth toroidal Fano symmetric varieties. More precisely, the idea of the proof is to proceed as follows: 1) prove that for any non-Fano symmetric variety $X$ and any blow-up $X^{\prime}$ of $X, X^{\prime}$ is not Fano (generalizing $(*)$ ); 2) find an explicit set of non-Fano varieties $\left\{X_{i}\right\}$ such that there are finitely many symmetric varieties that do not dominate any $\left.X_{i} ; 3\right)$ study these last varieties.

Unfortunately this strategy is too naive. Actually, property $(*)$ is false already in rank three. Indeed, let $\bar{Z}$ be the 3 -dimensional toric variety whose fan $\bar{\Delta}$ has 
maximal cones cone $\left(e_{1}, e_{2}, e_{3}\right)$ and cone $\left(e_{1}, e_{2}, e_{1}+e_{2}-e_{3}\right)$, where $\left\{e_{1}, e_{2}, e_{3}\right\}$ is any basis of $\chi_{*}(S)$. The function associated to its anticanonical bundle is linear, so $-K_{\bar{Z}}$ is nef but non-ample. Furthermore, the blow-up $\bar{Z}^{\prime}$ of $\bar{Z}$ along cone $\left(e_{1}, e_{2}\right)$ is Fano. We can take $\bar{Z} \times \mathbb{A}^{m}$ as higher dimensional example.

In the previous example, we have considered a blow-up along a subvariety with strictly positive dimension. In Section 4 we will prove a property similar to $(*)$ considering only blow-ups along compact orbits, i.e. $S$-fixed points. This property will allow us to prove the following classification, which in particular implies Theorem B. We also prove a more precise result when the rank is three (see the next section).

Theorem 2.2. Let $G / H$ be a symmetric space of rank $l(>1)$ associated to an involution $\theta$ and let $X$ be a symmetric variety obtained from the wonderful compactification $X_{0}$ of $G / H$ by a sequence of blow-ups along closed orbits.

Table 3. Fano $X_{1, \ldots, l}$

\begin{tabular}{cccc}
\hline$G^{\prime} / H^{\prime}$ & $\theta \mid G^{\prime}$ & $\operatorname{rank}(G / H)$ & $\operatorname{rank}\left(G^{\prime} / H^{\prime}\right)$ \\
\hline$S L_{3}$ & $A_{2}$ & $l=2$ & 2 \\
$S p i n_{5}$ & $B_{2}$ & $l=2$ & 2 \\
$S L_{6} / N\left(S p_{6}\right)$ & $A I I$ & $l=3$ & 2 \\
$S L_{n+1} / S\left(G L_{2} \times G L_{n-1}\right), n \geq 4$ & $A I I I$ & $l=2$ & 2 \\
$S p_{2 n} /\left(S p_{4} \times S p_{2 n-4}\right), n \geq 5$ & $C I I$ & $l \leq 3$ & 2 \\
$S p_{8} /\left(S p_{4} \times S p_{4}\right)$ & $C I I$ & $l=3$ & 2 \\
$S p_{8} / N\left(S p_{4} \times S p_{4}\right)$ & $C I I$ & $l=2$ & 2 \\
$S O_{10} / G L_{5}$ & $D I I I$ & $l \leq 3$ & 2 \\
$E_{6} / N\left(D_{5} \times \mathbb{C}^{*}\right)$ & $E I I I$ & $l \leq 4$ & 2 \\
$E_{6} / N\left(F_{4}\right)$ & $E I V$ & $l \leq 5$ & 2 \\
$P S L_{2}$ & $A_{1}$ & $l=2$ & 1 \\
$S L_{2}$ & $A 1$ & $l \leq 3$ & 1 \\
$S L_{n+1} / N\left(S O_{n}\right)$ & $A I$ & $l=2$ & 1 \\
$S L_{n+1} / S O_{n}$ & $A I$ & $l=2$ & 1 \\
$S L_{6} / N\left(S p_{6}\right)$ & $A I I$ & $l \leq 3$ & 1 \\
$S L_{6} / S p_{6}$ & $A I I$ & $l \leq 5$ & 1 \\
$S L_{n+1} / N\left(S\left(G L_{1} \times G L_{n}\right)\right)$ & $A I V$ & $l \leq n+1$ & 1 \\
$S O_{2 n+1} / N\left(S O_{1} \times S O_{2 n}\right)$ & $B I I$ & $l \leq n+1$ & 1 \\
$S O_{2 n+1} /\left(S O_{1} \times S O_{2 n}\right)$ & $B I I$ & $l \leq 2 n$ & 1 \\
$S p_{2 n} /\left(S p_{2} \times S p_{2 n-2}\right)$ & $C I I$ & $l \leq 2 n$ & 1 \\
$S O_{2 n} / N\left(S O_{1} \times S O_{2 n-1}\right)$ & $D I I$ & $l \leq n$ & 1 \\
$S O_{2 n} /\left(S O_{1} \times S O_{2 n-1}\right)$ & $D I I$ & $l \leq 2 n-1$ & 1 \\
$F_{4} / B_{4}$ & $F I I$ & $l \leq 12$ & 1 \\
\hline & & &
\end{tabular}


1. If $X$ is a Fano variety then it is either the wonderful variety $X_{0}$ or the blow-up $X_{1, \ldots, l}$ of $X_{0}$ along the closed orbit.

2. If there is an indecomposable factor of $(G, \theta)$ of rank at least 3 then $X_{1, \ldots, l}$ is not Fano.

3. If $(G, \theta)$ has rank at least 6 and has an indecomposable factor of rank 2, then $X_{1, \ldots, l}$ is not Fano.

4. If $X_{1, \ldots, l}$ is Fano, the possibilities for the indecomposable factors of $G / H$ are as in Table 3 (we also indicate the conditions on $\operatorname{rank}(G / H)$ so that such a factor can appear).

\section{§2.3. Fano symmetric varieties of rank 3}

In this section we suppose $X_{0}$ is wonderful of rank three; recall that $\left\{e_{1}, e_{2}, e_{3}\right\}$ is the basis of $\chi_{*}(S)$ which generates $C^{-}$. We classify all the Fano symmetric varieties obtainable from $X_{0}$ by a succession of blow-ups along $G$-subvarieties. This class of varieties contains many varieties; indeed each compact symmetric variety is dominated by a smooth toroidal variety obtained by a succession of blow-ups along $G$-subvarieties of codimension two (see [dCoPr85, Proposition 2.4]).

Given a toric variety $Z$ (above $\mathbb{A}^{3}$ ) that does not contain a subvariety isomorphic to $\bar{Z}$ we will prove a property very similar to $(*)$ (see Lemma 5.1). Instead, if $Z$ contains $\bar{Z}$ we will prove that it is sufficient to study the blow-up of $Z$ along the subvariety corresponding to cone $\left(e_{1}, e_{2}\right)$ (see Lemma 5.3). These lemmas allow us to prove the following classification, which implies Theorem E. We state separately the classification depending on whether $(G, \theta)$ is indecomposable, is a product of two indecomposable involutions, or a product of three involutions. We introduce the following notation:

- $X_{i j}$ is the blow-up of $X_{0}$ along (cone $\left.\left(e_{i}, e_{j}\right), \emptyset\right)$;

- $X_{123}$ is the blow-up of $X_{0}$ along the closed $G$-orbit;

- $X_{123, i j}$ is the blow-up of $X_{123}$ along $\left(\operatorname{cone}\left(e_{i}, e_{j}\right), \emptyset\right)$;

- $X_{123, i}$ is the blow-up of $X_{123}$ along (cone $\left.\left(e_{i}, e_{1}+e_{2}+e_{3}\right), \emptyset\right)$.

By [Ru09, Corollary 2.1], if $(G, \theta)=\left(G_{1}, \theta\right) \times\left(G_{2}, \theta\right)$ and $X_{0}$ is wonderful, then $H=H_{1} \times H_{2}$, where $H_{i}:=H \cap G_{i}$. Given a 1-rank symmetric space $G_{i} / H_{i}$, let $\psi_{i}(r):=-2 \delta+2 \delta_{0}-r e_{i}^{*}, m_{i}:=\max \left\{r: \psi_{i}(r)\left(\alpha_{i}^{\vee}\right)<0\right\}$ and $\bar{m}_{i}:=\max \{r:$ $\left.\psi_{i}(r)\left(\alpha_{i}^{\vee}\right) \leq 0\right\}$. Table 4 gives the values of $m_{i}$ and $\bar{m}_{i}$ for the various $G_{i} / H_{i}$. Moreover, we indicate by $e$ (resp. $h$ ) when $G_{i} / H_{i}$ is exceptional (resp. Hermitian non-exceptional). 
Table 4 . Weights of rank 1 symmetric spaces

\begin{tabular}{ccccc}
\hline$G / H$ & $\theta$ & $\bar{m}_{1}$ & $m_{1}$ & \\
\hline$P S L_{2}$ & $A_{1}$ & 1 & 0 & \\
$S L_{2}$ & $A_{1}$ & 2 & 1 & \\
$S L_{2} / N\left(S O_{2}\right)$ & $A I$ & 0 & 0 & $h$ \\
$S L_{2} / S O_{2}$ & $A I$ & 1 & 0 & $h$ \\
$S L_{2} / N\left(S p_{2}\right)$ & $A I I$ & 2 & 1 & \\
$S L_{2} / S p_{2}$ & $A I I$ & 4 & 3 & \\
$S L_{n} / S\left(G L_{1} \times G L_{n-1}\right)$ & $A I V$ & $n$ & $n-1$ & $e$ \\
$S O_{2 n+1} / N\left(S O_{1} \times S O_{2 n}\right)$ & $B I I$ & $n-1$ & $n-1$ & \\
$S O_{2 n+1} /\left(S O_{1} \times S O_{2 n}\right)$ & $B I I$ & $2 n-1$ & $2 n-2$ & \\
$S p_{2 n} /\left(S p_{2} \times S p_{2 n-2}\right)$ & $C I I$ & $2 n-1$ & $2 n-2$ & \\
$S O_{2 n} / S\left(O_{1} \times O_{2 n-1}\right)$ & $D I I$ & $n-1$ & $n-2$ & \\
$S O_{2 n} /\left(S O_{1} \times S O_{2 n-1}\right)$ & $D I I$ & $2 n-2$ & $2 n-3$ & \\
$F_{4} / B_{4}$ & $F I I$ & 11 & 10 & \\
\hline
\end{tabular}

Table 5. Fano indecomposable symmetric varieties of rank 3

\begin{tabular}{|c|c|c|}
\hline$G / H$ & $\theta$ & $X$ \\
\hline $\mathrm{PSL}_{4}$ & $A_{3}$ & $X_{0}, X_{13}$ \\
\hline $\mathrm{SO}_{7}$ & $B_{3}$ & $X_{0}$ \\
\hline$P S p_{6}$ & $C_{3}$ & $X_{0}, X_{13}$ \\
\hline$S p_{6}$ & $C_{3}$ & $X_{0}, X_{13}$ \\
\hline $\mathrm{SL}_{4} / \mathrm{N}\left(\mathrm{SO}_{4}\right)$ & $A I$ & $X_{0}$ \\
\hline$S L_{8} / N\left(S p_{8}\right)$ & $A I I$ & $X_{0}, X_{12}, X_{13}, X_{23}, X_{123,13}$ \\
\hline$S L_{n+1} / S\left(L_{3} \times L_{n-2}\right), n \geq 6$ & $A I I I$ & $X_{0}, X_{13}$ \\
\hline$S L_{6} / N\left(S\left(L_{3} \times L_{3}\right)\right)$ & $A I I I$ & $X_{0}$ \\
\hline$S L_{6} / S\left(L_{3} \times L_{3}\right)$ & $A I I I$ & $X_{0}$ \\
\hline$S O_{2 n+1} / N\left(S_{3} \times S O_{2 n-2}\right)$ & $B I$ & $X_{0}$ \\
\hline$S p_{6} / N\left(G L_{3}\right)$ & $C I$ & $\nexists$ \\
\hline$S p_{6} / G L_{3}$ & $C I$ & $X_{0}$ \\
\hline$S p_{12} / N\left(S p_{6} \times S p_{6}\right)$ & $C I I$ & $X_{0}, X_{12}, X_{13}, X_{23}$ \\
\hline$S p_{12} /\left(S p_{6} \times S p_{6}\right)$ & $C I I$ & $X_{0}, X_{12}, X_{13}, X_{23}, X_{123,13}$ \\
\hline$S p_{2 n} /\left(S p_{6} \times S p_{2 n-6}\right), n>6$ & $C I I$ & $X_{0}, X_{12}, X_{13}, X_{23}, X_{123,13}$ \\
\hline $\mathrm{SO}_{2 n} / \mathrm{N}\left(\mathrm{SO}_{3} \times \mathrm{SO}_{2 n-3}\right)$ & $D I$ & $X_{0}$ \\
\hline$S O_{12} / N\left(G L_{6}\right)$ & $D I I I$ & $X_{0}, X_{12}$ \\
\hline$S O_{12} / N\left(G L_{6}\right)$ & $D I I I$ & $X_{0}, X_{12}$ \\
\hline$S O_{14} / G L_{7}$ & $D I I I$ & $X_{0}, X_{12}, X_{13}, X_{23}, X_{123,13}$ \\
\hline$E_{7} / N\left(E_{6} \times \mathbb{C}^{*}\right)$ & $E V I I$ & $X_{0}, X_{12}$ \\
\hline$E_{7} /\left(E_{6} \times \mathbb{C}^{*}\right)$ & $E V I I$ & $X_{0}, X_{12}$ \\
\hline
\end{tabular}


Theorem 2.3. Let $G / H$ be an indecomposable symmetric space of rank 3 such that its standard compactification $X_{0}$ is wonderful. If $X$ is a smooth Fano compactification of $G / H$ obtained from $X_{0}$ by a sequence of blow-ups along $G$-subvarieties, then it is $X_{0}, X_{12}, X_{13}, X_{23}$ or $X_{123,13}$. More precisely, the Fano ones are those appearing in Table 5.

Theorem 2.4. Let $G / H$ be a symmetric space such that $X_{0}$ is wonderful and $(G, \theta)=\left(G_{1}, \theta\right) \times\left(G_{2}, \theta\right)$ with $\operatorname{rank}\left(G_{i} / G_{i}^{\theta}\right)=i$. If $X$ is a smooth Fano compactification of $G / H$ obtained from $X_{0}$ by a sequence of blow-ups along $G$-subvarieties, then it is $X_{0}, X_{12}, X_{13}, X_{23}, X_{123}, X_{123,12}, X_{123,13}, X_{123,23}, X_{123,1}, X_{123,12}$ or $X_{123,3}$. More precisely, the classification of such varieties is as in Table 6 . In the second column, we indicate the conditions on $m_{1}$ so that $X$ is Fano.

Theorem 2.5. Let $G / H$ be a symmetric space such that $X_{0}$ is wonderful. Suppose that $(G, \theta)=\left(G_{1}, \theta\right) \times\left(G_{2}, \theta\right) \times\left(G_{3}, \theta\right)$ with $\operatorname{rank}\left(G_{i} / G_{i}^{\theta}\right)=1$ and let $x_{r}$ be the number of factors $G_{i}$ such that $\psi_{i}(r)$ is antidominant and regular. If $X$ is a smooth Fano compactification of $G / H$ obtained from $X_{0}$ by a sequence of blow-ups along $G$ subvarieties, then it is $X_{0}, X_{12}, X_{13}, X_{23}, X_{123}, X_{123,12}, X_{123,13}, X_{123,23}, X_{123,1}$, $X_{123,2}$ or $X_{123,3}$. More precisely, we have the following classification (depending on $G / H)$ :

- If $x_{1} \leq 1$, then the smooth Fano compactifications of $G / H$ are $X_{0}, X_{12}, X_{13}$ and $X_{23}$. In particular, there are four of them.

- If $x_{1}=2$, then there are five Fano varieties. Let $i<j$ be the indices such that $\psi_{i}(1)$ and $\psi_{j}(1)$ are anti-dominant and regular. The smooth Fano compactifications of $G / H$ are $X_{0}, X_{12}, X_{13}, X_{23}$ and $X_{123, i j}$.

- If $\left(x_{1}, x_{2}\right)$ is to $(3,0)$ or $(3,1)$, then there are eight Fano varieties: $X_{0}, X_{12}$, $X_{13}, X_{23}, X_{123}, X_{123,12}, X_{123,13}$ and $X_{123,23}$.

- If $\left(x_{1}, x_{2}\right)=(3,2)$, then there are nine Fano varieties. Suppose that, for all $j \neq i, \psi_{j}(2)$ is anti-dominant and regular. Then the Fano varieties are $X_{0}, X_{12}$, $X_{13}, X_{23}, X_{123}, X_{123,12}, X_{123,13}, X_{123,23}$ and $X_{123, i}$.

- If $\left(x_{1}, x_{2}\right)=(3,3)$, then there are eleven Fano varieties: $X_{0}, X_{12}, X_{13}, X_{23}$, $X_{123}, X_{123,12}, X_{123,13}, X_{123,23}, X_{123,1}, X_{123,2}$ and $X_{123,3}$.

We will see that a weaker statement holds in a more general context.

Corollary 2.1. Given any wonderful $G$-variety $X_{0}$ of rank 3 (even non-symmetric) and any Fano variety $X$ obtained from $X_{0}$ by a succession of blow-ups along $G$-stable subvarieties, then $X$ is $X_{0}, X_{12}, X_{13}, X_{23}, X_{123}, X_{123,12}, X_{123,13}$, $X_{123,23}, X_{123,1}, X_{123,2}$ or $X_{123,3}$. 
Table 6. Fano decomposable symmetric varieties of rank 3

\begin{tabular}{|c|c|c|}
\hline$G_{2} / H_{2}$ & $m_{1}$ & $X$ \\
\hline$P S L_{3}$ & - & $X_{0}, X_{12}, X_{13}, X_{23}$ \\
\hline $\mathrm{SO}_{5}$ & - & $X_{0}, X_{12}$ \\
\hline $\operatorname{Spin}_{5}$ & - & $X_{0}, X_{12}, X_{13}, X_{23}$ \\
\hline$G_{2}$ & - & $X_{0}, X_{13}$ \\
\hline$S L_{3} / N\left(S O_{3}\right)$ & - & $X_{0}$ \\
\hline$S L_{6} / N\left(S p_{6}\right)$ & $\begin{array}{c}- \\
m_{1} \geq 1\end{array}$ & $\begin{array}{c}X_{0}, X_{12}, X_{13}, X_{23}, X_{123,23} \\
X_{123}, X_{123,12}, X_{123,13}\end{array}$ \\
\hline $\begin{array}{c}S L_{n+1} / S\left(G L_{2} \times G L_{n-1}\right) \\
(n \geq 5)\end{array}$ & $\begin{array}{c}- \\
m_{1} \geq 1\end{array}$ & $\begin{array}{c}X_{0}, X_{12}, X_{13}, X_{23} \\
X_{123,13}\end{array}$ \\
\hline$S L_{5} / S\left(G L_{2} \times G L_{3}\right)$ & - & $X_{0}, X_{12}, X_{13}, X_{23}$ \\
\hline$S L_{4} / N\left(S\left(G L_{2} \times G L_{2}\right)\right)$ & - & $X_{0}$ \\
\hline$S L_{4} / S\left(G L_{2} \times G L_{2}\right)$ & - & $X_{0}, X_{12}$ \\
\hline $\mathrm{SO}_{5} / \mathrm{S}\left(\mathrm{O}_{2} \times \mathrm{O}_{3}\right)$ & - & $X_{0}$ \\
\hline $\mathrm{SO}_{5} /\left(\mathrm{SO}_{2} \times \mathrm{SO}_{3}\right)$ & - & $X_{0}$ \\
\hline$S O_{2 n+1} / S\left(O_{2} \times O_{2 n-1}\right)$ & - & $X_{0}, X_{13}$ \\
\hline$S O_{2 n+1} /\left(S_{2} \times S O_{2 n-1}\right)$ & - & $X_{0}, X_{13}$ \\
\hline $\begin{array}{c}S p_{2 n} /\left(S p_{4} \times S p_{2 n-4}\right) \\
(n \geq 5)\end{array}$ & $\begin{array}{c}- \\
m_{1} \geq 1 \\
m_{1} \geq 2\end{array}$ & $\begin{array}{c}X_{0}, X_{12}, X_{13}, X_{23}, X_{123,23} \\
X_{123}, X_{123,12}, X_{123,13} \\
X_{123,2}\end{array}$ \\
\hline$S p_{8} / N\left(S p_{4} \times S p_{4}\right)$ & - & $X_{0}, X_{12}, X_{13}, X_{23}$ \\
\hline$S p_{8} /\left(S p_{4} \times S p_{4}\right)$ & $\begin{array}{c}- \\
m_{1} \geq 1\end{array}$ & $\begin{array}{c}X_{0}, X_{12}, X_{13}, X_{23}, X_{123,23} \\
X_{123}, X_{123,12}, X_{123,13}\end{array}$ \\
\hline$S O_{2 n} / S\left(O_{2} \times O_{2 n-2}\right)$ & - & $X_{0}, X_{13}$ \\
\hline$S_{2 n} / S O_{2} \times S O_{2 n-2}$ & - & $X_{0}, X_{13}$ \\
\hline$S O_{8} / N\left(G L_{4}\right)$ & - & $X_{0}, X_{12}$ \\
\hline $\mathrm{SO}_{8} / G L_{4}$ & - & $X_{0}, X_{12}$ \\
\hline$S O_{10} / G L_{5}$ & $\begin{array}{c}- \\
m_{1} \geq 1\end{array}$ & $\begin{array}{c}X_{0}, X_{12}, X_{13}, X_{23}, X_{123,23} \\
X_{123}, X_{123,12}, X_{123,13}\end{array}$ \\
\hline$E_{6} /\left(D_{5} \times \mathbb{C}^{*}\right)$ & $\begin{array}{c}- \\
m_{1} \geq 1 \\
m_{1} \geq 2\end{array}$ & $\begin{array}{c}X_{0}, X_{12}, X_{13}, X_{23}, X_{123,23} \\
X_{123}, X_{123,12}, X_{123,13}, X_{123,1} \\
X_{123,2}, X_{123,3}\end{array}$ \\
\hline$E_{6} / N\left(F_{4}\right)$ & $\begin{array}{c}- \\
m_{1} \geq 1 \\
m_{1} \geq 2\end{array}$ & $\begin{array}{c}X_{0}, X_{12}, X_{13}, X_{23}, X_{123,23} \\
X_{123}, X_{123,12}, X_{123,13}, X_{123,1} \\
X_{123,2}, X_{123,3}\end{array}$ \\
\hline$G_{2} /\left(A_{1} \times A_{1}\right)$ & - & $\nexists$ \\
\hline
\end{tabular}




\section{$\S 2.4$. (Quasi-) Fano symmetric varieties of rank 2}

Finally we state the results about symmetric varieties of rank two. We define $\psi_{i}(r)$, $m_{i}$ and $\bar{m}_{i}$ as in the previous section. Note that, supposing $R_{G, \theta}=A_{1} \times A_{1}$, the $m_{i}$ have the same value if $\chi_{*}(S)=\mathbb{Z}\left(\omega_{1}^{\vee}+\omega_{2}^{\vee}\right) \oplus \mathbb{Z} 2 \omega_{2}^{\vee}$ or if $H=G^{\theta}$. Moreover, $G / H=\prod G_{i} /\left(H \cap G_{i}\right)$ if $H$ is either $G^{\theta}$ or $N_{G}\left(G^{\theta}\right)$. So to find the values of the $m_{i}$ it is sufficient to check Table 4.

Notice that to unequivocally specify a projective 2-rank symmetric variety with $\rho$ injective over $\varrho^{-1}(\varrho(D(X))$, it is sufficient to give $\Delta[p]$. Indeed, we have seen in $\S 1.5$ that if $\rho$ is injective over $\varrho^{-1}(\varrho(D(X))$ then $\Delta$ determines $\mathcal{F}(X)$. Moreover, $\Delta$ is specified by the maximal cones, which have maximal dimension if $X$ is projective. When the rank is 2, the maximal cones are cone $(v, w)$ with $v, w \in \Delta[p]$ and such that: (i) $v \neq w$; (ii) $\operatorname{cone}(v, w) \cap \Delta[p]=\{v, w\}$; (iii) the interior of cone $(v, w)$ intersects $C^{-}$.

2.4.1. Fano symmetric varieties. First we consider the locally factorial (resp. smooth) Fano symmetric varieties with $G$ semisimple.

Theorem 2.6. Let $G / H$ be a symmetric space of rank 2 (with $G$ semisimple).

- If a (projective) symmetric variety $X$ is Fano then $\rho^{-1}(\rho(D(X)))=D(X)$. If moreover $X$ is locally factorial, then $\sharp \rho(D(X))=\sharp \rho^{-1}(\rho(D(X)))$.

- If $R_{G, \theta}$ is irreducible and $X$ is a Fano locally factorial equivariant compactification of $G / H$, we have exactly the following possibilities for $\Delta[p]$ :

1. $\Delta[p]=\left\{e_{1}, \alpha_{1}^{\vee}\right\}$ if $\sharp \rho^{-1}\left(\alpha_{1}^{\vee}\right)=1$ and $R_{G, \theta}$ is not $G_{2}$;

2. $\Delta[p]=\left\{e_{2}, \alpha_{2}^{\vee}\right\}$ if $\sharp \rho^{-1}\left(\alpha_{2}^{\vee}\right)=1$ and $R_{G, \theta}$ is not $B_{2}$;

3. $\Delta[p]=\left\{e_{2}, \alpha_{2}^{\vee}\right\}$ if $R_{G, \theta}=B_{2}$ and $H=G^{\theta}$;

4. $\Delta[p]=\left\{-\omega_{1}^{\vee},-\omega_{2}^{\vee}\right\}$ if $G / H \neq G_{2} /\left(S L_{2} \times S L_{2}\right)$ and $H=N\left(G^{\theta}\right)$;

5. $\Delta[p]=\left\{-2 \omega_{1}^{\vee},-\omega_{2}^{\vee}\right\}$ if $R_{G, \theta}=B_{2}$ and $H=G^{\theta}$;

6. $\Delta[p]=\left\{\alpha_{1}^{\vee}, \alpha_{2}^{\vee},-\alpha_{1}^{\vee}-\alpha_{2}^{\vee}\right\}$ if $R_{G, \theta}=A_{2}$ and $H=G^{\theta}$;

7. $\Delta[p]=\left\{\alpha_{1}^{\vee},-\omega_{1}^{\vee}-\omega_{2}^{\vee},-3 \omega_{1}^{\vee}\right\}$ (and $\left.\Delta[p]=\left\{\alpha_{2}^{\vee},-\omega_{1}^{\vee}-\omega_{2}^{\vee},-3 \omega_{2}^{\vee}\right\}\right)$ if $H=$ $G^{\theta}$ and $R_{G, \theta}=A_{2}$;

8. $\Delta[p]=\left\{\alpha_{1}^{\vee},-\omega_{1}^{\vee}-\omega_{2}^{\vee},-4 \omega_{1}^{\vee}-\omega_{2}^{\vee},-3 \omega_{1}^{\vee}\right\}$ (and $\Delta[p]=\left\{\alpha_{2}^{\vee},-\omega_{1}^{\vee}-\omega_{2}^{\vee},-\omega_{1}^{\vee}-\right.$ $\left.\left.4 \omega_{2}^{\vee},-3 \omega_{2}^{\vee}\right\}\right)$ if $H=G^{\theta}, R_{G, \theta}=A_{2}$ and $\theta \neq-\mathrm{id}$ over $\chi_{*}(T)$;

9. $\Delta[p]=\left\{e_{1}, e_{2}, e_{1}+e_{2}\right\}$ if $\chi_{*}(S)=\mathbb{Z} e_{1} \oplus \mathbb{Z} e_{2}$ and $-2 \delta+2 \delta_{0}+e_{i}^{*} \in \operatorname{int}\left(C^{-}\right)$ for each $i$.

The previous varieties are singular in the following cases:

$\circ \Delta[p]=\left\{e_{i}, \alpha_{i}^{\vee}\right\}$ and $i=1,2$ if $R_{G, \theta}=A_{2}$; 
$\circ \Delta[p]=\left\{e_{1}, \alpha_{1}^{\vee}\right\}$ if $R_{G, \theta}=B_{2}, H=G^{\theta}($ and $\sharp D(G / H)=2)$;

○ $\Delta[p]=\left\{e_{2}, \alpha_{2}^{\vee}\right\}$ if $R_{G, \theta}=B C_{2}$ (and $\left.\sharp D(G / H)=2\right)$.

- Suppose $(G, \theta)=\left(G_{1}, \theta\right) \times\left(G_{2}, \theta\right)$ and $\chi_{*}(S)=\mathbb{Z} e_{1} \oplus \mathbb{Z} e_{2}$; we have the following locally factorial Fano varieties:

1. $\Delta[p]=\left\{e_{1}, e_{2}\right\}$;

2. $\Delta[p]=\left\{e_{1}, e_{2}, e_{1}+e_{2}\right\}$;

3. $\Delta[p]=\left\{\alpha_{1}^{\vee},-\alpha_{1}^{\vee},-r \alpha_{1}^{\vee}+e_{2}\right\}$ if $r \leq m_{2}+1$ and $\sharp \rho^{-1}\left(\alpha_{1}^{\vee}\right)=1$;

4. $\Delta[p]=\left\{\alpha_{2}^{\vee},-\alpha_{2}^{\vee}, e_{1}-r \alpha_{2}^{\vee}\right\}$ if $r \leq m_{1}+1$ and $\sharp \rho^{-1}\left(\alpha_{2}^{\vee}\right)=1$;

5. $\Delta[p]=\left\{\alpha_{1}^{\vee},-\alpha_{1}^{\vee},-r \alpha_{1}^{\vee}+e_{2},-(r+1) \alpha_{1}^{\vee}+e_{2}\right\}$ if $r \leq m_{2} \quad$ and $\sharp \rho^{-1}\left(\alpha_{1}^{\vee}\right)=1$;

6. $\Delta[p]=\left\{\alpha_{2}^{\vee},-\alpha_{2}^{\vee}, e_{1}-r \alpha_{2}^{\vee}, e_{1}-(r+1) \alpha_{2}^{\vee}\right\}$ if $r \leq m_{1}$ and $\sharp \rho^{-1}\left(\alpha_{2}^{\vee}\right)=1$;

7. $\Delta[p]=\left\{\alpha_{1}^{\vee}, \alpha_{2}^{\vee},-\alpha_{1}^{\vee}-\alpha_{2}^{\vee}\right\}$ if $\sharp D(G / H)=2$.

Only the first two are smooth.

- If $G / H$ is decomposable and $\chi_{*}(S)=\mathbb{Z}\left(\frac{1}{2} \alpha_{1}^{\vee}+\frac{1}{2} \alpha_{2}^{\vee}\right) \oplus \mathbb{Z} \alpha_{2}^{\vee}$, we have the following locally factorial Fano compactifications of $G / H$ :

1. $\Delta[p]=\left\{\alpha_{1}^{\vee}, \alpha_{2}^{\vee},-\frac{1}{2} \alpha_{1}^{\vee}-\frac{1}{2} \alpha_{2}^{\vee}\right\}$;

2. $\Delta[p]=\left\{\alpha_{1}^{\vee},-\alpha_{1}^{\vee},-\frac{2 r+1}{2} \alpha_{1}^{\vee}-\frac{1}{2} \alpha_{2}^{\vee}\right\}$ if $0 \leq r \leq \frac{m_{2}+1}{2}$;

3. $\Delta[p]=\left\{\alpha_{2}^{\vee},-\alpha_{2}^{\vee},-\frac{1}{2} \alpha_{1}^{\vee}-\frac{2 r+1}{2} \alpha_{2}^{\vee}\right\}$ if $0 \leq r \leq \frac{m_{1}+1}{2}$;

4. $\Delta[p]=\left\{\alpha_{1}^{\vee},-\alpha_{1}^{\vee},-\frac{2 r+1}{2} \alpha_{1}^{\vee}-\frac{1}{2} \alpha_{2}^{\vee},-\frac{2 r+3}{2} \alpha_{1}^{\vee}-\frac{1}{2} \alpha_{2}^{\vee}\right\}$ if $0 \leq r \leq \frac{m_{2}-1}{2}$;

5. $\Delta[p]=\left\{\alpha_{2}^{\vee},-\alpha_{2}^{\vee},-\frac{1}{2} \alpha_{1}^{\vee}-\frac{2 r+1}{2} \alpha_{2}^{\vee},-\frac{1}{2} \alpha_{1}^{\vee}-\frac{2 r+3}{2} \alpha_{2}^{\vee}\right\}$ if $0 \leq r \leq \frac{m_{1}-1}{2}$.

The first one is smooth, while the other ones are smooth if and only if $r=0$.

Proposition 2.1. Let $G / H$ be a symmetric space of rank 2 with $X_{0}$ smooth. If $\theta$ is indecomposable, the Fano toroidal compactifications of $G / H$ are as in Table 7. If $\theta$ is decomposable, the Fano toroidal compactifications of $G / H$ are the following:

- $\Delta[p]=\left\{e_{1}, e_{2}\right\}$;

- $\Delta[p]=\left\{e_{1}, e_{2}, r e_{1}+e_{2}\right\}$ with $r \leq m_{2}+1$;

- $\Delta[p]=\left\{e_{1}, e_{2}, e_{1}+r e_{2}\right\}$ with $r \leq m_{1}+1$.

2.4.2. Smooth quasi-Fano varieties. Now, we consider the smooth (resp. locally factorial) quasi-Fano symmetric varieties with rank two and $G$ semisimple. A Gorenstein (projective) variety is called quasi-Fano if its anticanonical divisor is big and nef. 
Table 7. Fano toroidal indecomposable symmetric varieties with rank 2

\begin{tabular}{|c|c|c|c|}
\hline$G / H$ & $\theta$ & $\Delta[p]$ & $\Delta[p]$ \\
\hline$P S L_{3}$ & $A_{2}$ & $\left\{-\omega_{1}^{\vee},-\omega_{2}^{\vee}\right\}$ & $\left\{-\omega_{1}^{\vee},-\omega_{2}^{\vee},-\omega_{1}^{\vee}-\omega_{2}^{\vee}\right\}$ \\
\hline $\mathrm{SO}_{3}$ & $B_{2}$ & $\left\{-\omega_{1}^{\vee},-\omega_{2}^{\vee}\right\}$ & \\
\hline $\operatorname{Spin}_{3}$ & $B_{2}$ & $\left\{-2 \omega_{1}^{\vee},-\omega_{2}^{\vee}\right\}$ & $\left\{-2 \omega_{1}^{\vee},-\omega_{2}^{\vee},-2 \omega_{1}^{\vee}-\omega_{2}^{\vee}\right\}$ \\
\hline$G_{2}$ & $G_{2}$ & $\left\{-\omega_{1}^{\vee},-\omega_{2}^{\vee}\right\}$ & \\
\hline$S L_{3} / N\left(S O_{3}\right)$ & $A I$ & $\left\{-\omega_{1}^{\vee},-\omega_{2}^{\vee}\right\}$ & \\
\hline$S L_{6} / N\left(S p_{6}\right)$ & $A I I$ & $\begin{array}{c}\left\{-\omega_{1}^{\vee},-\omega_{2}^{\vee}\right\} \\
\left\{-\omega_{1}^{\vee},-\omega_{2}^{\vee},-\omega_{1}^{\vee}-\omega_{2}^{\vee}\right\}\end{array}$ & $\begin{array}{l}\left\{-\omega_{1}^{\vee},-\omega_{2}^{\vee},-2 \omega_{1}^{\vee}-\omega_{2}^{\vee}\right\} \\
\left\{-\omega_{1}^{\vee},-\omega_{2}^{\vee},-\omega_{1}^{\vee}-2 \omega_{2}^{\vee}\right\}\end{array}$ \\
\hline$S L_{n+1} / S\left(G L_{2} \times G L_{n-1}\right)$ & AIII & $\left\{-\omega_{1}^{\vee},-\omega_{2}^{\vee}\right\}$ & $\begin{array}{c}\left\{-\omega_{1}^{\vee},-\omega_{2}^{\vee},-r \omega_{1}^{\vee}-\omega_{2}^{\vee}\right\}, \\
r \leq n-3\end{array}$ \\
\hline$S L_{4} / N\left(S\left(G L_{2} \times G L_{2}\right)\right)$ & AIII & $\left\{-\omega_{1}^{\vee},-\omega_{2}^{\vee}\right\}$ & \\
\hline$S L_{4} / S\left(G L_{2} \times G L_{2}\right)$ & AIII & $\left\{-\omega_{1}^{\vee},-2 \omega_{2}^{\vee}\right\}$ & \\
\hline$S O_{2 n+1} / S\left(O_{2} \times O_{2 n-1}\right)$ & $B I$ & $\left\{-\omega_{1}^{\vee},-\omega_{2}^{\vee}\right\}$ & \\
\hline$S_{2 n+1} / S_{2} \times S O_{2 n-1}$ & $B I$ & $\left\{-2 \omega_{1}^{\vee},-\omega_{2}^{\vee}\right\}$ & \\
\hline$S p_{2 n} /\left(S p_{4} \times S p_{2 n-4}\right)$ & $C I I$ & $\left\{-\omega_{1}^{\vee},-\omega_{2}^{\vee}\right\}$ & $\begin{array}{c}\left\{-\omega_{1}^{\vee},-\omega_{2}^{\vee},-r \omega_{1}^{\vee}-\omega_{2}^{\vee}\right\}, \\
r \leq 2 n-6\end{array}$ \\
\hline$n \geq 5$ & & $\left\{-\omega_{1}^{\vee},-\omega_{2}^{\vee},-\omega_{1}^{\vee}-2 \omega_{2}^{\vee}\right\}$ & \\
\hline$S p_{8} / N\left(S p_{4} \times S p_{4}\right)$ & $C I I$ & $\left\{-\omega_{1}^{\vee},-\omega_{2}^{\vee}\right\}$ & $\left\{-\omega_{1}^{\vee},-\omega_{2}^{\vee},-\omega_{1}^{\vee}-\omega_{2}^{\vee}\right\}$ \\
\hline$S p_{8} /\left(S p_{4} \times S p_{4}\right)$ & $C I I$ & $\begin{array}{c}\left\{-\omega_{1}^{\vee},-2 \omega_{2}^{\vee}\right\} \\
\left\{-\omega_{1}^{\vee},-2 \omega_{2}^{\vee},-\omega_{1}^{\vee}-2 \omega_{2}^{\vee}\right\}\end{array}$ & $\begin{array}{c}\left\{-\omega_{1}^{\vee},-2 \omega_{2}^{\vee},-2 \omega_{1}^{\vee}-2 \omega_{2}^{\vee}\right\} \\
\left\{-\omega_{1}^{\vee},-2 \omega_{2}^{\vee},-\omega_{1}^{\vee}-4 \omega_{2}^{\vee}\right\}\end{array}$ \\
\hline$S O_{2 n} / S\left(O_{2} \times O_{2 n-2}\right)$ & $D I$ & $\left\{-\omega_{1}^{\vee},-\omega_{2}^{\vee}\right\}$ & \\
\hline$S_{2 n} /\left(S_{2} \times S O_{2 n-2}\right)$ & $D I$ & $\left\{-2 \omega_{1}^{\vee},-\omega_{2}^{\vee}\right\}$ & \\
\hline $\mathrm{SO}_{8} / \mathrm{N}\left(\mathrm{SO}_{4}\right)$ & $D I I I$ & $\left\{-\omega_{1}^{\vee},-\omega_{2}^{\vee}\right\}$ & \\
\hline $\mathrm{SO}_{8} / \mathrm{SO}_{4}$ & $D I I I$ & $\left\{-\omega_{1}^{\vee},-2 \omega_{2}^{\vee}\right\}$ & \\
\hline $\mathrm{SO}_{10} / \mathrm{SO}_{5}$ & $D I I I$ & $\begin{array}{c}\left\{-\omega_{1}^{\vee},-\omega_{2}^{\vee}\right\} \\
\left\{-\omega_{1}^{\vee},-\omega_{2}^{\vee},-\omega_{1}^{\vee}-2 \omega_{2}^{\vee}\right\}\end{array}$ & $\begin{array}{c}\left\{-\omega_{1}^{\vee},-\omega_{2}^{\vee},-\omega_{1}^{\vee}-\omega_{2}^{\vee}\right\} \\
\left\{-\omega_{1}^{\vee},-\omega_{2}^{\vee},-2 \omega_{1}^{\vee}-\omega_{2}^{\vee}\right\}\end{array}$ \\
\hline$E_{6} /\left(D_{5} \times \mathbb{C}^{*}\right)$ & EIII & $\begin{array}{c}\left\{-\omega_{1}^{\vee},-\omega_{2}^{\vee},-\omega_{1}^{\vee}-r \omega_{2}^{\vee}\right\} \\
r \leq 3 \\
\left\{-\omega_{1}^{\vee},-\omega_{2}^{\vee}\right\}\end{array}$ & $\begin{array}{c}\left\{-\omega_{1}^{\vee},-\omega_{2}^{\vee},-r \omega_{1}^{\vee}-\omega_{2}^{\vee}\right\}, \\
r \leq 4\end{array}$ \\
\hline$E_{6} / N\left(F_{4}\right)$ & $E I V$ & $\begin{array}{c}\left\{-\omega_{1}^{\vee},-\omega_{2}^{\vee}\right\} \\
\left\{-\omega_{1}^{\vee},-\omega_{2}^{\vee},-r \omega_{1}^{\vee}-\omega_{2}^{\vee}\right\} \\
r \leq 4\end{array}$ & $\left\{\begin{array}{c}\left\{-\omega_{1}^{\vee},-\omega_{2}^{\vee},-\omega_{1}^{\vee}-r \omega_{2}^{\vee}\right\}, \\
r \leq 4\end{array}\right.$ \\
\hline$G_{2} /\left(A_{1} \times A_{1}\right)$ & $G$ & $\nexists$ & \\
\hline
\end{tabular}


Theorem 2.7. Let $G / H$ be a symmetric space of rank 2 (with $G$ semisimple).

- The nefness of the anticanonical bundle of a compactification of $G / H$ depends only on the fan associated to the colored fan (and not on the whole colored fan).

- The fans of the locally factorial quasi-Fano (but non-Fano) compactifications of an indecomposable symmetric space of rank 2 (with $G$ semisimple) are those in Table 8 (we also have to require that $\rho$ is injective over $D(X)$ ). Such a variety is singular if and only if $\rho(D(X))$ contains an exceptional root.

Table 8. Quasi-Fano indecomposable symmetric varieties with rank 2

\begin{tabular}{|c|c|c|}
\hline$G / H$ & $\theta$ & $\Delta[p]$ \\
\hline$P S L_{3}$ & $\left(A_{2}\right)$ & $\nexists$ \\
\hline$S L_{3}$ & $\left(A_{2}\right)$ & $\left\{\alpha_{i}^{\vee},-\omega_{i}^{\vee}-\omega_{i^{c}}^{\vee},-4 \omega_{i}^{\vee}-\omega_{i^{c}}^{\vee},-5 \omega_{i}^{\vee}-2 \omega_{i^{c}}^{\vee},-3 \omega_{i}^{\vee}\right\}, i=1,2$ \\
\hline $\mathrm{SO}_{5}$ & $\left(B_{2}\right)$ & $\left\{-\omega_{1}^{\vee},-\omega_{2}^{\vee},-\omega_{1}^{\vee}-\omega_{2}^{\vee}\right\}$ \\
\hline $\operatorname{Spin}_{5}$ & $\left(B_{2}\right)$ & $\left\{-2 \omega_{1}^{\vee},-\omega_{2}^{\vee},-2 \omega_{1}^{\vee}-\omega_{2}^{\vee},-2 \omega_{1}^{\vee}-2 \omega_{2}^{\vee}\right\}$ \\
\hline$G_{2}$ & $\left(G_{2}\right)$ & $\nexists$ \\
\hline$S L_{3} / N\left(S O_{3}\right)$ & $A I$ & $\left\{-\omega_{1}^{\vee},-\omega_{2}^{\vee},-\omega_{1}^{\vee}-\omega_{2}^{\vee}\right\}$ \\
\hline$S L_{3} / S O_{3}$ & $A I$ & $\left\{\alpha_{i}^{\vee},-3 \omega_{i}^{\vee},-\omega_{i}^{\vee}-\omega_{i^{c}}^{\vee},-4 \omega_{i}^{\vee}-\omega_{i^{c}}^{\vee}\right\}, i=1,2$ \\
\hline$S L_{6} / N\left(S p_{6}\right)$ & $A I I$ & $\left\{-\omega_{1}^{\vee},-\omega_{2}^{\vee},-\omega_{i}^{\vee}-\omega_{i^{c}}^{\vee},-2 \omega_{i}^{\vee}-\omega_{i^{c}}^{\vee}\right\}, i=1,2$ \\
\hline$S L_{6} / S p_{6}$ & $A I I$ & $\begin{array}{c}\left\{\alpha_{i}^{\vee},-\omega_{i}^{\vee}-\omega_{i^{c}}^{\vee}, \ldots,-(r+3) \omega_{i}^{\vee}-r \omega_{i^{c}}^{\vee},-3 \omega_{i}^{\vee}\right\} \\
i=1,2 ; r=2,3,4\end{array}$ \\
\hline & & $\left\{\alpha_{i}^{\vee},-\omega_{i}^{\vee}-\omega_{i^{c}}^{\vee},-4 \omega_{i}^{\vee}-\omega_{i^{c}}^{\vee},-7 \omega_{i}^{\vee}-\omega_{i^{c}}^{\vee},-3 \omega_{i}^{\vee}\right\}, i=1,2$ \\
\hline $\begin{array}{c}S L_{n+1} / S\left(G L_{2} \times G L_{n-1}\right) \\
n \geq 4\end{array}$ & $A I I I$ & $\begin{array}{c}\left\{-\omega_{1}^{\vee},-\omega_{2}^{\vee},-\omega_{1}^{\vee}-\omega_{2}^{\vee}, \ldots,-r \omega_{1}^{\vee}-\omega_{2}^{\vee}\right\}, 2 \leq r \leq n-2 \\
\left\{\alpha_{2}^{\vee},-\omega_{2}^{\vee}\right\}\end{array}$ \\
\hline$S L_{4} / N\left(S\left(G L_{2} \times G L_{2}\right)\right)$ & $A I I I$ & $\left\{-\omega_{1}^{\vee},-\omega_{2}^{\vee},-\omega_{1}^{\vee}-\omega_{2}^{\vee}\right\}$ \\
\hline$S L_{4} / S\left(G L_{2} \times G L_{2}\right)$ & AIII & $\begin{array}{c}\left\{-\omega_{1}^{\vee},-2 \omega_{2}^{\vee},-\omega_{1}^{\vee}-2 \omega_{2}^{\vee}\right\} \\
\left\{\alpha_{2}^{\vee},-2 \omega_{2}^{\vee}\right\}\end{array}$ \\
\hline$S O_{2 n+1} / S\left(O_{2} \times O_{2 n-1}\right)$ & $B I$ & $\left\{-\omega_{1}^{\vee},-\omega_{2}^{\vee},-\omega_{1}^{\vee}-\omega_{2}^{\vee}, \ldots,-r \omega_{1}^{\vee}-\omega_{2}^{\vee}\right\}, r \leq n-2 ; n \geq 3$ \\
\hline $\mathrm{SO}_{5} / \mathrm{S}\left(\mathrm{O}_{2} \times \mathrm{O}_{3}\right)$ & $B I$ & $\nexists$ \\
\hline$S O_{2 n+1} /\left(S O_{2} \times S O_{2 n-1}\right)$ & $B I$ & $\begin{aligned}\left\{-2 \omega_{1}^{\vee},-\omega_{2}^{\vee},-2 \omega_{1}^{\vee}-\right. & \left.\omega_{2}^{\vee}, \ldots,-2 r \omega_{1}^{\vee}-\omega_{2}^{\vee}\right\}, r \leq n-1 \\
& \left\{\alpha_{1}^{\vee},-\omega_{1}^{\vee}\right\}\end{aligned}$ \\
\hline $\begin{array}{l}S p_{2 n} /\left(S p_{4} \times S p_{2 n-4}\right) \\
\quad n>4\end{array}$ & $C I I$ & $\begin{array}{c}\left\{-\omega_{1}^{\vee},-\omega_{2}^{\vee},-\omega_{1}^{\vee}-\omega_{2}^{\vee}, \ldots,-r \omega_{1}^{\vee}-\omega_{2}^{\vee}\right\}, 2 \leq r \leq n-5 \\
\left\{-\omega_{1}^{\vee},-\omega_{2}^{\vee},-\omega_{1}^{\vee}-\omega_{2}^{\vee},-\omega_{1}^{\vee}-2 \omega_{2}^{\vee}\right\}\end{array}$ \\
\hline$S p_{8} / N\left(S p_{4} \times S p_{4}\right)$ & $C I I$ & $\begin{array}{l}\left\{-\omega_{1}^{\vee},-\omega_{2}^{\vee},-\omega_{1}^{\vee}-\omega_{2}^{\vee},-2 \omega_{1}^{\vee}-\omega_{2}^{\vee}\right\} \\
\left\{-\omega_{1}^{\vee},-\omega_{2}^{\vee},-\omega_{1}^{\vee}-\omega_{2}^{\vee},-\omega_{1}^{\vee}-2 \omega_{2}^{\vee}\right\}\end{array}$ \\
\hline$S p_{8} /\left(S p_{4} \times S p_{4}\right)$ & $C I I$ & $\begin{array}{c}\left\{-\omega_{1}^{\vee},-2 \omega_{2}^{\vee},-\omega_{1}^{\vee}-2 \omega_{2}^{\vee}, \ldots,-r \omega_{1}^{\vee}-2 \omega_{2}^{\vee}\right\}, 2 \leq r \leq 3 \\
\left\{-\omega_{1}^{\vee},-2 \omega_{2}^{\vee},-\omega_{1}^{\vee}-2 \omega_{2}^{\vee},-\omega_{1}^{\vee}-4 \omega_{2}^{\vee}\right\}\end{array}$ \\
\hline$S O_{2 n} / S\left(O_{2} \times O_{2 n-2}\right)$ & $D I$ & $\left\{-\omega_{1}^{\vee},-\omega_{2}^{\vee},-\omega_{1}^{\vee}-\omega_{2}^{\vee}, \ldots,-r \omega_{1}^{\vee}-\omega_{2}^{\vee}\right\}, r \leq n-2$ \\
\hline $\mathrm{SO}_{2 n} /\left(\mathrm{SO}_{2} \times \mathrm{SO}_{2 n-2}\right)$ & $D I$ & $\begin{array}{c}\left\{-2 \omega_{1}^{\vee},-\omega_{2}^{\vee},-2 \omega_{1}^{\vee}-\omega_{2}^{\vee}, \ldots,-2 r \omega_{1}^{\vee}-\omega_{2}^{\vee}\right\}, r \leq n-2 \\
\left\{\alpha_{1}^{\vee},-2 \omega_{1}^{\vee}\right\}\end{array}$ \\
\hline
\end{tabular}




\begin{tabular}{|c|c|c|}
\hline $\mathrm{SO}_{8} / \mathrm{N}\left(\mathrm{SO}_{4}\right)$ & $D I I I$ & $\begin{array}{c}\left\{-\omega_{1}^{\vee},-\omega_{2}^{\vee},-\omega_{1}^{\vee}-\omega_{2}^{\vee}\right\} \\
\left\{-\omega_{1}^{\vee},-\omega_{2}^{\vee},-\omega_{1}^{\vee}-\omega_{2}^{\vee},-\omega_{1}^{\vee}-2 \omega_{2}^{\vee}\right\}\end{array}$ \\
\hline $\mathrm{SO}_{8} / \mathrm{SO}_{4}$ & $D I I I$ & $\begin{array}{c}\left\{-\omega_{1}^{\vee},-2 \omega_{2}^{\vee}, \ldots,-\omega_{1}^{\vee}-2 r \omega_{2}^{\vee}\right\}, r=1,2 \\
\left\{\alpha_{2}^{\vee},-2 \omega_{2}^{\vee}\right\}\end{array}$ \\
\hline$S O_{10} / S O_{5}$ & $D I I I$ & $\begin{array}{c}\left\{-\omega_{1}^{\vee},-\omega_{2}^{\vee},-\omega_{1}^{\vee}-\omega_{2}^{\vee}, \ldots,-r \omega_{1}^{\vee}-\omega_{2}^{\vee}\right\}, r=2,3 \\
\left\{-\omega_{1}^{\vee},-\omega_{2}^{\vee},-\omega_{1}^{\vee}-\omega_{2}^{\vee},-\omega_{1}^{\vee}-2 \omega_{2}^{\vee}\right\} \\
\left\{\alpha_{2}^{\vee},-\omega_{2}^{\vee}\right\}\end{array}$ \\
\hline$E_{6} /\left(D_{5} \times \mathbb{C}^{*}\right)$ & EIII & $\begin{array}{c}\left\{-\omega_{1}^{\vee},-\omega_{2}^{\vee},-\omega_{1}^{\vee}-\omega_{2}^{\vee}, \ldots,-r \omega_{1}^{\vee}-\omega_{2}^{\vee}\right\}, 2 \leq r \leq 5 \\
\left\{-\omega_{1}^{\vee},-\omega_{2}^{\vee},-\omega_{1}^{\vee}-\omega_{2}^{\vee}, \ldots,-\omega_{1}^{\vee}-r \omega_{2}^{\vee}\right\}, 2 \leq r \leq 3 \\
\left\{\alpha_{2}^{\vee},-\omega_{2}^{\vee}\right\}\end{array}$ \\
\hline$E_{6} / N\left(F_{4}\right)$ & $E I V$ & $\begin{array}{c}\left\{-\omega_{1}^{\vee},-\omega_{2}^{\vee},-\omega_{i}^{\vee}-\omega_{i^{c}}^{\vee}, \ldots,-r \omega_{i}^{\vee}-\omega_{i^{c}}^{\vee}\right\} \\
i=1,2 ; r=2,3,4\end{array}$ \\
\hline$E_{6} / F_{4}$ & $E I V$ & $\begin{array}{c}\left\{\alpha_{i}^{\vee},-\omega_{i}^{\vee}-\omega_{i^{c}}^{\vee}, \ldots,-(r+3) \omega_{i}^{\vee}-r \omega_{i^{c}}^{\vee},-3 \omega_{i}^{\vee}\right\}, \\
i=1,2 ; r=2, \ldots, 8 \\
\left\{\alpha_{i}^{\vee},-\omega_{i}^{\vee}-\omega_{i^{c}}^{\vee}, \ldots,-(3 r+1) \omega_{i}^{\vee}-\omega_{i^{c}}^{\vee},-3 \omega_{i}^{\vee}\right\}, \\
\quad i=1,2 ; r=2,3,4\end{array}$ \\
\hline$G_{2} /\left(A_{1} \times A_{1}\right)$ & $G$ & $\left\{-\omega_{1}^{\vee},-\omega_{2}^{\vee}\right\}$ \\
\hline
\end{tabular}

- If $(G, \theta)=\left(G_{1}, \theta\right) \times\left(G_{2}, \theta\right)$, and $\chi_{*}(S)=\mathbb{Z} e_{1} \oplus \mathbb{Z} e_{2}$, let $v_{j}(i):=-i \alpha_{j}^{\vee}+e_{j^{c}}$ and $w_{j}(x, y):=-(x y+1) \alpha_{j}^{\vee}+y e_{j^{c}}$. We have the following locally factorial quasi-Fano compactifications of $G / H$, which are not Fano (we always suppose $\rho$ injective over $D(X))$ :

1. $\Delta[p]=\left\{\alpha_{1}^{\vee},-\alpha_{1}^{\vee}, v_{1}(r), v_{1}(r+1), \ldots, v_{1}(r+s)\right\}$ if (i) $s=0,1$, (ii) $r+s \leq$ $\bar{m}_{2}+1$, and (iii) either $r+s>m_{2}+1$ or $\sharp \rho^{-1}\left(\alpha_{1}^{\vee}\right)=2$;

2. $\Delta[p]=\left\{\alpha_{2}^{\vee},-\alpha_{2}^{\vee}, v_{2}(r), v_{2}(r+1), \ldots, v_{2}(r+s)\right\}$ if (i) $s=0,1$, (ii) $r+s \leq$ $\bar{m}_{1}+1$, and (iii) either $r+s>m_{1}+1$ or $\sharp \rho^{-1}\left(\alpha_{2}^{\vee}\right)=2$;

3. $\Delta[p]=\left\{\alpha_{1}^{\vee},-\alpha_{1}^{\vee}, v_{1}(r), v_{1}(r+1), \ldots, v_{1}(r+s)\right\}$ if (i) $s \geq 2$ and (ii) $r+s \leq$ $\bar{m}_{2}+1$;

4. $\Delta[p]=\left\{\alpha_{2}^{\vee},-\alpha_{2}^{\vee}, v_{2}(r), v_{2}(r+1), \ldots, v_{2}(r+s)\right\}$ if (i) $s \geq 2$ and (ii) $r+s \leq$ $\bar{m}_{1}+1$;

5. $\Delta[p]=\left\{\alpha_{1}^{\vee},-\alpha_{1}^{\vee},-r \alpha_{1}^{\vee}+e_{2}, w_{1}(r, 1), \ldots, w_{1}(r, s)\right\}$ if $r \leq \bar{m}_{2}$ and $2 \leq s \leq$ $\bar{m}_{1}+1$;

6. $\Delta[p]=\left\{\alpha_{2}^{\vee},-\alpha_{2}^{\vee},-r \alpha_{2}^{\vee}+e_{1}, w_{2}(r, 1), \ldots, w_{2}(r, s)\right\}$ if $r \leq \bar{m}_{1}$ and $2 \leq s \leq$ $\bar{m}_{2}+1$;

7. $\Delta[p]=\left\{\alpha_{1}^{\vee}, \alpha_{2}^{\vee},-\alpha_{1}^{\vee}-\alpha_{2}^{\vee}\right\}$ if $\sharp D(G / H)>2$ and $H=G^{\theta}$;

8. $\left.\Delta[p]=\left\{e_{1}, e_{1}+e_{2}, e_{1}+2 e_{2}, \ldots, e_{1}+(s-1) e_{2}, e_{1}+s e_{2}, e_{2}\right)\right\}$ if $2 \leq s \leq \bar{m}_{1}+1$;

9. $\left.\Delta[p]=\left\{e_{2}, e_{1}+e_{2}, 2 e_{1}+e_{2}, \ldots,(s-1) e_{1}+e_{2}, s e_{1}+e_{2}, e_{1}\right)\right\}$ if $2 \leq s \leq \bar{m}_{2}+1$. 
These varieties are smooth if they are toroidal or if, for all $\alpha^{\vee} \in \rho(D(X))$, $\sharp \rho^{-1}\left(\alpha^{\vee}\right)=2$ and $2 \alpha \notin R_{G, \theta}$.

- If $G / H$ is decomposable and $\chi_{*}(S)=\mathbb{Z}\left(\frac{1}{2} \alpha_{1}^{\vee}+\frac{1}{2} \alpha_{2}^{\vee}\right) \oplus \mathbb{Z} \alpha_{2}^{\vee}$, let $v_{j}(i):=-\frac{2 i+1}{2} \alpha_{j}^{\vee}$ $-\frac{1}{2} \alpha_{j^{c}}^{\vee}$ and $w_{j}(x, y):=-\frac{2 x y+y+2}{2} \alpha_{j}^{\vee}-\frac{y}{2} \alpha_{j^{c}}^{\vee}$. We have the following locally factorial quasi-Fano compactifications of $G / H$, which are not Fano:

1. $\Delta[p]=\left\{\alpha_{1}^{\vee},-\alpha_{1}^{\vee}, v_{1}(r), v_{1}(r+1), \ldots, v_{1}(r+s)\right\}$ if (i) $r \geq 0$, (ii) $s \geq 2$ and (iii) $r+s \leq \frac{\bar{m}_{2}+1}{2}$;

2. $\Delta[p]=\left\{\alpha_{2}^{\vee},-\alpha_{2}^{\vee}, v_{2}(r), v_{2}(r+1), \ldots, v_{2}(r+s)\right\}$ if (i) $r \geq 0$, (ii) $s \geq 2$ and (iii) $r+s \leq \frac{\bar{m}_{1}+1}{2}$;

3. $\Delta[p]=\left\{\alpha_{1}^{\vee},-\alpha_{1}^{\vee}, v_{1}(r), v_{1}(r+1), \ldots, v_{1}(r+s)\right\}$ if (i) $r \geq 0$, (ii) $s=0,1$ and (iii) $\frac{m_{2}+1}{2}<r+s \leq \frac{\bar{m}_{2}+1}{2}$;

4. $\Delta[p]=\left\{\alpha_{2}^{\vee},-\alpha_{2}^{\vee}, v_{2}(r), v_{2}(r+1), \ldots, v_{2}(r+s)\right\}$ if (i) $r \geq 0$, (ii) $s=0,1$ and (iii) $\frac{m_{1}+1}{2}<r+s \leq \frac{\bar{m}_{1}+1}{2}$;

5. $\Delta[p]=\left\{\alpha_{1}^{\vee},-\alpha_{1}^{\vee},-\frac{2 r+1}{2} \alpha_{1}^{\vee}-\frac{1}{2} \alpha_{2}^{\vee}, w_{1}(r, 1), \ldots, w_{1}(r, s)\right\}$ if (i) $r \leq \frac{\bar{m}_{2}-1}{2}$ and (ii) $2 \leq s \leq \bar{m}_{1}+1$;

6. $\Delta[p]=\left\{\alpha_{2}^{\vee},-\alpha_{2}^{\vee},-\frac{1}{2} \alpha_{1}^{\vee}-\frac{2 r+1}{2} \alpha_{2}^{\vee}, w_{2}(r, 1), \ldots, w_{2}(r, s)\right\}$ if (i) $r \leq \frac{\bar{m}_{1}-1}{2}$ and (ii) $2 \leq s \leq \bar{m}_{2}+1$;

7. $\Delta[p]=\left\{-\alpha_{1}^{\vee},-\alpha_{2}^{\vee},-\frac{1}{2} \alpha_{1}^{\vee}-\frac{1}{2} \alpha_{2}^{\vee}\right\}$.

The last variety is smooth, while the other ones are smooth if and only if $r=0$.

2.4.3. Symmetric Fano varieties with $G$ reductive. In this section we consider the 2-rank locally factorial Fano symmetric varieties over which a group $G$ acts that is only reductive. If $G$ is a torus, then $X$ is the projective space. So, we can suppose $G=G^{\prime} \times \mathbb{C}^{*}$ with $H \cap \mathbb{C}^{*}=\left(\mathbb{C}^{*}\right)^{\theta}=\{ \pm$ id $\}$. Write $\chi_{*}\left(\mathbb{C}^{*} /\{ \pm\right.$ id $\left.\}\right)=\mathbb{Z} f$.

If $R_{G, \theta}=B C_{1}$, then $H=G^{\theta}$ and $\chi_{*}(S)=\mathbb{Z} f \oplus \mathbb{Z} \alpha^{\vee}$. Instead, if $R_{G, \theta}=A_{1}$ there are three possibilities: 1) $\chi_{*}(S)=\mathbb{Z} f \oplus \mathbb{Z} \alpha^{\vee}$ and $\left.H=G^{\theta} ; 2\right) \chi_{*}(S)=$ $\mathbb{Z} f \oplus \mathbb{Z} \frac{1}{2} \alpha^{\vee}$ and $\left.H=N_{G^{\prime}}\left(G^{\theta}\right) \times\{ \pm 1\} ; 3\right) \chi_{*}(S)=\mathbb{Z} f \oplus \mathbb{Z} \frac{\alpha^{\vee}+f}{2}$. In the last case $H$ is generated by $G^{\theta}$ and by $n_{1} n_{2}$, where $n_{1} \in N_{G^{\prime}}\left(\left(G^{\prime}\right)^{\theta}\right) \backslash\left(G^{\prime}\right)^{\theta}$ and $n_{2} \in \mathbb{C}^{*}$ has order four; in particular $\left[G^{\theta}: H\right]=2$. Let $e$ be the primitive positive multiple of $-\alpha^{\vee}$ and let $\left\{e^{*}, f^{*}\right\}$ be the dual basis of $\{e, f\}$.

Theorem 2.8. Let $G / H$ be a symmetric space of rank two such that $G$ is neither semisimple nor abelian. As before, write $\psi(r)=-2 \delta+2 \delta_{0}-r e^{*}$ and $m_{1}:=\max \{r$ : $\psi(r)<0\}$. The Fano locally factorial compactifications of $G / H$ are the following:

1. $\Delta[p]=\{f,-f, e+r f\}$ if $\chi_{*}(S)=\mathbb{Z} e \oplus \mathbb{Z} f, r \in \mathbb{Z}, r \leq m_{1}+1$ and $-r \leq m_{1}+1$;

2. $\Delta[p]=\{f,-f, e+r f, e+(r+1) f\}$ if $\chi_{*}(S)=\mathbb{Z} e \oplus \mathbb{Z} f, r \in \mathbb{Z}, r \leq m_{1}$ and $-r \leq m_{1}+1$; 
3. $\Delta[p]=\left\{\alpha^{\vee},-f,-\alpha^{\vee}+f\right\}$ (and $\left.\Delta[p]=\left\{\alpha^{\vee}, f,-\alpha^{\vee}-f\right\}\right)$ if $\chi_{*}(S)=\mathbb{Z} \alpha^{\vee} \oplus \mathbb{Z} f$ and $\sharp D(G / H)=1$;

4. $\Delta[p]=\left\{\alpha^{\vee},-f,-\alpha^{\vee},-\alpha^{\vee}+f\right\}$ (and $\left.\Delta[p]=\left\{\alpha^{\vee}, f,-\alpha^{\vee},-\alpha^{\vee}-f\right\}\right)$ if $\chi_{*}(S)$ $=\mathbb{Z} \alpha^{\vee} \oplus \mathbb{Z} f$ and $\sharp D(G / H)=1$;

5. $\Delta[p]=\left\{f,-f,-\frac{1}{2} \alpha^{\vee}+\frac{2 r+1}{2} f\right\}$ if $\chi_{*}(S)=\mathbb{Z} f \oplus \mathbb{Z}\left(\frac{\alpha^{\vee}+f}{2}\right), r \in \mathbb{Z}, r \leq \frac{m_{1}+1}{2}$ and $-r \leq \frac{m_{1}+3}{2}$;

6. $\Delta[p]=\left\{f,-f,-\frac{1}{2} \alpha^{\vee}+\frac{2 r+1}{2} f,-\frac{1}{2} \alpha^{\vee}+\frac{2 r+3}{2} f\right\}$ if $\chi_{*}(S)=\mathbb{Z} f \oplus \mathbb{Z}\left(\frac{\alpha^{\vee}+f}{2}\right)$, $r \in \mathbb{Z}, r \leq \frac{m_{1}-1}{2}$ and $-r \leq \frac{m_{1}+3}{2}$;

7. $\Delta[p]=\left\{\alpha^{\vee},-\frac{1}{2} \alpha^{\vee}+\frac{1}{2} f,-\frac{1}{2} \alpha^{\vee}-\frac{1}{2} f,-\alpha^{\vee}\right\}$ if $\chi_{*}(S)=\mathbb{Z} f \oplus \mathbb{Z}\left(\frac{\alpha^{\vee}+f}{2}\right)$;

8. $\Delta[p]=\left\{\alpha^{\vee},-\frac{1}{2} \alpha^{\vee}+\frac{1}{2} f,-\frac{1}{2} \alpha^{\vee}-\frac{1}{2} f\right\}$ if $\chi_{*}(S)=\mathbb{Z} f \oplus \mathbb{Z}\left(\frac{\alpha^{\vee}+f}{2}\right)$;

9. $\Delta[p]=\left\{\alpha^{\vee},-f,-\frac{1}{2} \alpha^{\vee}+\frac{1}{2} f\right\}$ (and $\left.\Delta[p]=\left\{\alpha^{\vee}, f,-\frac{1}{2} \alpha^{\vee}-\frac{1}{2} f\right\}\right)$ if $\chi_{*}(S)=$ $\mathbb{Z} f \oplus \mathbb{Z}\left(\frac{\alpha^{\vee}+f}{2}\right) ;$

10. $\Delta[p]=\left\{\alpha^{\vee},-f,-\frac{1}{2} \alpha^{\vee}+\frac{1}{2} f,-\frac{1}{2} \alpha^{\vee}-\frac{1}{2} f\right\}$ (and $\Delta[p]=\left\{\alpha^{\vee}, f,-\frac{1}{2} \alpha^{\vee}+\frac{1}{2} f\right.$, $\left.\left.-\frac{1}{2} \alpha^{\vee}-\frac{1}{2} f\right\}\right)$ if $\chi_{*}(S)=\mathbb{Z} f \oplus \mathbb{Z}\left(\frac{\alpha^{\vee}+f}{2}\right)$.

The only singular varieties are the ones in cases 3 and 4 .

\section{$\S 3$. Standard symmetric varieties}

In this section we prove Theorem 2.1 (and Theorem A). Recall that the standard compactification $X_{0}$ of $G / H$ is the simple symmetric variety associated to the colored cone $\left(C^{-}, \emptyset\right)$, so $\widetilde{k}_{X_{0}}$ is always strictly convex.

Proof of Theorem 2.1. We can suppose $\theta$ is indecomposable (see Lemma 2.1). We have to determine when $\left(\widetilde{k}_{C^{-}}, \alpha_{i}\right) \leq 0$ for each $i \in\{1, \ldots, l\}$. We can write $-2 \delta+2 \delta_{0}$ as the sum of the spherical weights $-2 \widetilde{\delta}=-2 \sum_{\beta_{j} \in \bar{R}_{G}^{1}} \varpi_{j}$ and $2 \widetilde{\delta}_{0}=$ $-2 \sum_{\beta_{j} \in \bar{R}_{G}^{0}} \varpi_{j}+2 \delta_{0}$. Write $\beta_{j}-\theta\left(\beta_{j}\right)=\alpha_{i}$, so that $\left(\widetilde{k}_{C^{-}}, \alpha_{i}\right)=2\left(\widetilde{k}_{C^{-}}, \beta_{j}\right)$ and $\left(2 \widetilde{\delta}_{0}, \alpha_{i}\right)=4\left(\widetilde{\delta}_{0}, \beta_{j}\right) \leq 0$. Thus $\left(-2 \delta+2 \delta_{0}\right)\left(\alpha_{i}^{\vee}\right)=-1$ if $\omega_{i}=2 \varpi_{j}$ and $\left(-2 \delta+2 \delta_{0}\right)\left(\alpha_{i}^{\vee}\right) \leq-2$ otherwise. Suppose now $H$ is autonormalizing, i.e. $H=N\left(G^{\theta}\right)$; the case where $H \subsetneq N(H)$ is very similar. We want to study $k_{C^{-}}=-\sum_{i=1}^{l} \alpha_{i}$. By the expression of the Cartan matrix of $R_{G, \theta}, k_{C^{-}}\left(\alpha_{i}^{\vee}\right) \leq 1$ for each $i$. Therefore $\widetilde{k}_{C^{-}}$is always antidominant. If $\widetilde{k}_{C^{-}}$is not regular, then there is a (unique) $\alpha_{i}=2 \beta_{j} \in \bar{R}_{G, \theta}$ such that $k_{X}\left(\alpha_{i}^{\vee}\right)=1$; in particular $G$ is simple. By the classification of the involutions by their Satake diagrams, $\widetilde{k}_{C^{-}}$is not regular if and only if $\theta=-$ id over $\chi(S)$ and $R_{G, \theta}$ is different from $A_{l}$ and $B_{l}$. 


\section{$\S 4$. Blows-ups along closed orbits}

In this section we prove a partial result in arbitrary rank: Theorem 2.2 (and Theorem B). First, we prove a property similar to $(*)$ (see $\S 2.2$ ). Recall that we have associated a toric variety $Z$ to any toroidal symmetric variety $X$; moreover $-K_{Z}$ is ample if $X$ is Fano.

Lemma 4.1. Let $Z$ be a smooth $l$-dimensional toric variety whose fan contains two l-dimensional cones $\sigma_{+}$and $\sigma_{-}$such that: (i) $\sigma_{+} \cap \sigma_{-}$has dimension $l-1$ and (ii) $\sigma_{+} \cup \sigma_{-}$is strictly convex. Assume moreover that the piecewise linear function associated to the anticanonical bundle of $Z$ is not strictly convex on $\sigma_{+} \cup \sigma_{-}$. Then the anticanonical bundle of any toric variety obtained from $Z$ by a sequence of blow-ups centered at $S$-fixed points is not ample.

We can reformulate the first hypothesis in a more combinatorial way. Indeed, we can write $\sigma_{+}=\operatorname{cone}\left(v_{1}, \ldots, v_{l-1}, v_{+}\right)$and $\sigma_{-}=\operatorname{cone}\left(v_{1}, \ldots, v_{l-1}, v_{-}\right)$with $v_{1}, \ldots, v_{l-1}, v_{+}, v_{-}$primitive and $v_{+}+v_{-}=\sum a_{i} v_{i}$, where the $a_{i}$ are positive integers, not all zero.

Proof of Lemma 4.1. The anticanonical bundle of a variety with satisfies the hypotheses of the lemma is not ample. Thus, it is sufficient to show that the blow-up $Z^{\prime}$ of $Z$ centered in any $\sigma \in \Delta(l)$ satisfies again the hypotheses of the lemma. We can suppose $\sigma=\sigma_{+}$by symmetry. Then the fan of $Z^{\prime}$ contains $\sigma_{-}$and $\sigma^{\prime}:=\operatorname{cone}\left(v_{1}, \ldots, v_{l-1}, v_{+}+\sum v_{i}\right)$. We have $\left(v_{+}+\sum v_{i}\right)+v_{-}=\sum\left(a_{i}+1\right) v_{i}$, so $Z^{\prime}$ satisfies the hypotheses with respect to $\sigma_{-}$and $\sigma^{\prime}$.

Now we can classify the toric varieties with ample anticanonical bundle which are obtained from $\mathbb{A}^{l}$ by a sequence of blow-ups centered at $S$-fixed points.

Proposition 4.1. Let $Z$ be a smooth toric variety with ample anticanonical bundle which is obtained from $\mathbb{A}^{l}$ by a sequence of blow-ups centered at $S$-fixed points. Then it is either $\mathbb{A}^{l}$ or the blow-up of $\mathbb{A}^{l}$ at the $S$-fixed point.

Proof. It is not difficult to see that the blow-up $Z_{1}$ of $\mathbb{A}^{l}$ at the $S$-stable point has ample anticanonical bundle. The blow-up of $Z_{1}$ at the $S$-fixed point corresponding to cone $\left(e_{1}, \ldots, \hat{e}_{j}, \ldots, e_{l}, \sum e_{i}\right)$ satisfies the hypotheses of the previous lemma with respect to cone $\left(e_{1}, \ldots, \hat{e}_{h}, \ldots, e_{l}, \sum_{i=1}^{l} e_{i}\right)$ and cone $\left(e_{1}, \ldots, \hat{e}_{h}, \ldots, \hat{e}_{j}, \ldots, e_{l}\right.$, $\left.\sum_{i=1}^{l} e_{i}, 2 \sum_{i=1}^{l} e_{i}-e_{j}\right)$, where $h \neq j$.

Thus, a symmetric variety obtained from a wonderful one by a sequence of blow-ups along closed orbits can be Fano only if it is either the wonderful variety or its blow-up along the closed orbit (the restriction of the anticanonical bundle of the 
symmetric variety to the associated toric variety is the anticanonical bundle of this last variety by $\S 1.7)$. We have already considered the wonderful case. Now, we prove in particular that, when such a blow-up is Fano, the rank of every indecomposable factor of $R_{G, \theta}$ is at most 3 .

Lemma 4.2. Let $X_{1, \ldots, l}$ be the blow-up of the wonderful compactification of $G / H$ along the closed orbit and suppose that $R_{G, \theta}$ contains an irreducible factor of rank at least three. If $X_{1, \ldots, l}$ is Fano then it is indecomposable, has rank 3 and $H \subsetneq$ $N\left(G^{\theta}\right)$.

Proof. The weights $\left\{\widetilde{k}_{C}\right\}$ associated to $-\widetilde{K}_{X_{1, \ldots l}}$ are $\lambda_{i}=-2 \delta+2 \delta_{0}-(l-2) e_{i}^{*}+$ $\sum_{j \neq i} e_{j}^{*}$ with $i=1, \ldots, l$. First, suppose $G / H$ is indecomposable and write $e_{i}^{*}=$ $-x_{i} \alpha_{i}$. If $H \subsetneq N\left(G^{\theta}\right)$, then $R_{G, \theta}$ has type $A_{1}, B_{2}$ or $C_{l}$ because $X_{0}$ is wonderful (see the remark at the end of $\S 3$ ).

We consider two cases. First, suppose that there is $\beta_{h} \in \bar{R}_{G}^{1}$ orthogonal to $R_{G}^{0}$. Write $\beta_{h}-\theta\left(\beta_{h}\right)=\alpha_{j}$, so $0>\left(\lambda_{j}, \alpha_{j}^{\vee}\right)=\left(-2 \delta+2 \delta_{0}, \alpha_{j}^{\vee}\right)+\left((l-2) x_{j} \alpha_{j}^{\vee}, \alpha_{j}^{\vee}\right)-$ $\left(\sum_{i \neq j} x_{i} \alpha_{i}^{\vee}, \alpha_{j}^{\vee}\right) \geq-2+2(l-2) x_{j}+0$. Notice that we have used that $\left(\delta_{0}, \alpha_{j}^{\vee}\right)=0$. By the previous remark on $R_{G, \theta}$ we have $x_{j}^{-1} \leq 2$, so $l=3$ and $H \subsetneq N\left(G^{\theta}\right)$. Note that the $x_{i}$ are all 1 if $H=N\left(G^{\theta}\right)$.

Suppose now that there is not such a root. By the classification of indecomposable symmetric spaces, we have the following possibilities: 1) $\theta$ has type $A I I$ and $G / G^{\theta}$ is $\left.S L_{2 l+2} / S p_{2 l+2} ; 2\right) \theta$ has type $C I I$ and $G / G^{\theta}$ is $S p_{2 n} /\left(S p_{2 l} \times S p_{2 n-2 l}\right)$; 3) $\theta$ has type $D I I I$ and $G / G^{\theta}$ is $S O_{4 l+2} / G L_{2 l+1}$. Then there are $\beta_{3}, \beta_{5} \in R_{G}^{0}$ orthogonal to $\bar{R}_{G}^{0} \backslash\left\{\beta_{3}, \beta_{5}\right\}$ and $\beta_{4} \in R_{G}^{1}$ such that $\alpha_{2}=\beta_{3}+2 \beta_{4}+\beta_{5}$ (see $\$ 1.4$ ). Moreover, $\left(\beta_{3}, \beta_{3}\right)=\left(\beta_{4}, \beta_{4}\right)=\left(\beta_{5}, \beta_{5}\right), \alpha_{2}^{\vee}=\frac{1}{\left(\beta_{2}, \beta_{2}\right)} \alpha_{2}$ and $x_{i}=1$ if $i<l$. Thus $0>\left(\lambda_{2}, \alpha_{2}^{\vee}\right)=\left(-2 \delta+2 \delta_{0}, \alpha_{2}^{\vee}\right)+(l-2)\left(\alpha_{2}, \alpha_{2}^{\vee}\right)-\left(\alpha_{1}, \alpha_{2}^{\vee}\right)-x_{3}\left(\alpha_{3}, \alpha_{2}^{\vee}\right) \geq$ $-4+2(l-2)+1+x_{3}$, so we again have $l=3$ and $H \subsetneq N\left(G^{\theta}\right)$.

Finally, suppose $\theta$ is decomposable. Let $(G, \theta)=\left(G_{1}, \theta_{1}\right) \times\left(G_{2}, \theta_{2}\right)$ with $l^{\prime}:=\operatorname{rank}\left(G_{1} / G_{1}^{\theta}\right) \geq 3$ and define the weight $\lambda_{i}^{\prime}$ for $G_{1}$ analogously to $\lambda_{i}$. We have $\lambda_{i}=\lambda_{i}^{\prime}-\left(l-l^{\prime}\right) e_{i}^{*}+\omega$ where $\omega$ is orthogonal to $R_{G_{1}, \theta}$. By the previous part of the proof there is always an $i$ with $\lambda_{i}^{\prime}\left(\alpha_{i}^{\vee}\right) \geq-1$, so $\lambda_{i}\left(\alpha_{i}^{\vee}\right) \geq \lambda_{i}^{\prime}\left(\alpha_{i}^{\vee}\right)+\frac{1}{2}\left(\alpha_{i}, \alpha_{i}^{\vee}\right) \geq 0$, a contradiction.

One can prove Theorem 2.2 by an explicit analysis of the indecomposable involutions of rank at most three.

\section{$\S 5$. Regular Fano varieties of rank 3}

In this section we prove Theorems $2.3-2.5$. We begin by proving a result similar to Lemma 4.1 . Let $\widetilde{Z}$ be the toric variety whose fan $\widetilde{\Delta}$ has maximal cones 
cone $\left(v_{1}, v_{2}, v_{+}\right)$and cone $\left(v_{1}, v_{2}, v_{-}=x_{1} v_{1}+x_{2} v_{2}-v_{+}\right)$, where $\left\{v_{1}, v_{2}, v_{+}\right\}$is a basis of $\chi_{*}(S), x_{1}+x_{2}>0$ and $x_{1} \geq x_{2} \geq 0$. The anticanonical bundle of $\widetilde{Z}$ is ample if and only if $x_{1}=x_{1}+x_{2}=1$. In this case, $\widetilde{Z}$ is the blow-up of $\mathbb{A}^{3}$ along a stable subvariety of codimension 2. Moreover, the anticanonical bundle of $\widetilde{Z}$ is nef but non-ample if and only if $x_{1}+x_{2}=2$. We have two possibilities: either $v_{+}+v_{-}=v_{1}+v_{2}$ or $v_{+}+v_{-}=2 v_{1}$. In the first case we have a variety isomorphic to the variety $\bar{Z}$ of $\S 2.2$. This is the more problematic case, so we will study it later.

Lemma 5.1. Let $Z$ be a smooth 3-dimensional toric variety whose fan contains two maximal cones cone $\left(v_{1}, v_{2}, v_{+}\right)$and $\operatorname{cone}\left(v_{1}, v_{2}, v_{-}\right)$such that $v_{+}+v_{-}=x_{1} v_{1}+$ $x_{2} v_{2}$, where $x_{1}$ and $x_{2}$ are integers with $x_{1} \geq x_{2} \geq 0$. Suppose moreover that $Z$ satisfies the following condition:

$$
x_{1} \geq 2 .
$$

Then the anticanonical bundle of any toric variety $Z^{\prime}$ obtained from $Z$ by a sequence of blow-ups along $S$-subvarieties is not ample.

Proof. Note that the anticanonical bundle of $Z$ is not ample. We say that a variety satisfies weakly the hypotheses of the lemma if it satisfies the weaker condition

$$
x_{1}+x_{2} \geq 2 .
$$

We use the following trivial observation: $x_{1}+x_{2}>2$ implies $x_{1} \geq 2$. One can try to prove this lemma by induction as Lemma 4.1. Unfortunately we can only prove the following weaker statement.

Lemma 5.2. Let $Z$ be a toric variety which satisfies $(W H)$ and let $Z^{\prime}$ be the blow-up of $Z$ along a cone $\tau$.

1. If $\tau \neq$ cone $\left(v_{1}, v_{2}\right)$, then $Z^{\prime}$ satisfies $(H)$.

2. If $Z$ satisfies $(H)$, then $Z^{\prime}$ satisfies $(W H)$.

Proof. We can suppose $\tau \subset$ cone $\left(v_{1}, v_{2}, v_{-}\right)$by symmetry. If $\tau \neq \operatorname{cone}\left(v_{1}, v_{2}\right)$, we have three possibilities: $\tau=\operatorname{cone}\left(v_{1}, v_{-}\right), \tau=\operatorname{cone}\left(v_{2}, v_{-}\right)$or $\tau=\operatorname{cone}\left(v_{1}, v_{2}, v_{-}\right)$. We always have $\Delta_{Z^{\prime}}[p]=\Delta_{Z}[p] \cup\left\{v^{\prime}:=v_{-}+b_{1} v_{1}+b_{2} v_{2}\right\}$ with $b_{1}, b_{2} \in\{0,1\}$. Moreover, $\Delta_{Z^{\prime}}$ contains the cones cone $\left(v_{1}, v_{2}, v_{+}\right)$and cone $\left(v_{1}, v_{2}, v^{\prime}\right)$ and we have $v^{\prime}+v_{+}=\left(x_{1}+b_{1}\right) v_{1}+\left(x_{2}+b_{2}\right) v_{1}$ with $\left(x_{1}+b_{1}\right)+\left(x_{2}+b_{2}\right)>2$, so $Z^{\prime}$ satisfies $(H)$.

Finally let $\tau=\operatorname{cone}\left(v_{1}, v_{2}\right)$. The fan of $Z^{\prime}$ contains cone $\left(v_{1}, v_{1}+v_{2}, v_{+}\right)$and cone $\left(v_{1}, v_{1}+v_{2}, v_{-}\right)$. We have $v_{+}+v_{-}=\left(x_{1}-x_{2}\right) v_{1}+x_{2}\left(v_{1}+v_{2}\right)$ with $\left(x_{1}-x_{2}\right)+x_{2}$ $=x_{1} \geq 2$. 
Now, we consider the general case. We have a sequence $Z=Z_{0} \leftarrow Z_{1} \leftarrow$ $\cdots \leftarrow Z_{r}=Z^{\prime}$ where $Z_{i+1}$ is the blow-up of $Z_{i}$ along the cone $\tau_{i}$. Let $\Delta_{i}=\Delta_{Z_{i}}$ and let $j$ be maximal such that $Z_{j}$ satisfies $(H)$ (with respect to cone $\left(w_{1}, w_{2}, w_{+}\right.$) and cone $\left.\left(w_{1}, w_{2}, w_{-}\right)\right)$. By the previous lemma $Z_{j+1}$ satisfies $(W H)$, in particular its anticanonical bundle is not ample. By the maximality of $j, Z_{j+1}$ does not satisfy $(H)$, thus, by the previous lemma, $\tau_{j}=\operatorname{cone}\left(w_{1}, w_{2}\right)$ and $Z_{j+1}$ contains a variety isomorphic to $\bar{Z}$. By the previous proof, we can suppose that the maximal cones in the fan $\bar{\Delta}$ of that variety are $\sigma_{+}=\operatorname{cone}\left(w_{1}, w_{1}+w_{2}, w_{+}\right)$and $\sigma_{-}=\operatorname{cone}\left(w_{1}, w_{1}+w_{2}, w_{-}\right)$; in particular $Z_{j+1}$ satisfies $(W H)$ with respect to $\sigma_{+}$ and $\sigma_{-}$. Moreover $w_{+}+w_{-}=2 w_{1}+w_{2}$.

If $\Delta_{r}$ contains $\bar{\Delta}$ then $-K_{Z^{\prime}}$ is not ample. Indeed, otherwise there is a minimal $h$ such that $\bar{\Delta}$ is not contained in $\Delta_{h+1}$; in particular $\tau_{h}$ is contained in $\sigma^{+} \cup \sigma^{-}$. We claim that $Z_{h+1}$ satisfies $(H)$, which gives a contradiction.

By the first part of Lemma 5.2 we can suppose $\tau_{h}=\operatorname{cone}\left(w_{1}, w_{1}+w_{2}\right)$; otherwise $Z_{h+1}$ satisfies $(H)$. The fan of $Z_{h+1}$ contains two cones cone $\left(w_{1}+w_{2}\right.$, $\left.w_{+}, 2 w_{1}+w_{2}\right)$ and cone $\left(w_{1}+w_{2}, w_{+}, w^{\prime}\right)$, with $w^{\prime}=w_{2}+x_{1}\left(w_{1}+w_{2}\right)+x_{2} w_{+}$ and $x_{1}, x_{2} \geq 0$. Therefore $Z_{h+1}$ satisfies $(H)$ with respect to these cones. Indeed $\left(2 w_{1}+w_{2}\right)+w^{\prime}=\left(2+x_{1}\right)\left(w_{1}+w_{2}\right)+x_{2} w_{+}$.

Now we want to study the varieties that contain an open subvariety isomorphic to $\bar{Z}$. Observe that these varieties are never Fano varieties. Let $Z$ be such a variety and let $Z^{\prime}$ be the blow-up of $Z$ along the subvariety of $\bar{Z}$ associated to cone $\left(e_{1}, e_{2}\right)$. Such a subvariety is the unique $S$-stable projective curve contained in $\bar{Z}$. We prove that, if $Z^{\prime}$ satisfies $(H)$, then there does not exist a Fano variety obtainable from $Z$ by a sequence of blow-ups.

Lemma 5.3. Let $Z$ be a smooth 3-dimensional toric variety whose fan contains cone $\left(v_{1}, v_{2}, v_{3}\right)$ and cone $\left(v_{1}, v_{2}, v_{1}+v_{2}-v_{3}\right)$ for suitable $v_{1}, v_{2}, v_{3}$. Let $Z^{\prime}$ be the blow-up of $Z$ along the stable subvariety corresponding to cone $\left(v_{1}, v_{2}\right)$ and let $Z^{\prime \prime}$ be a toric variety obtained from $Z$ by a sequence of blow-ups along $S$-subvarieties. If the anticanonical bundle of $Z^{\prime \prime}$ is ample, then $Z^{\prime \prime}$ is obtainable from $Z^{\prime}$ by a sequence of blow-ups along $S$-subvarieties.

Proof. We cannot proceed as in the previous lemma, because we do not know the other cones of $\Delta_{Z}$. We again have a sequence $Z=Z_{0} \leftarrow Z_{1} \leftarrow \cdots \leftarrow Z_{h}=Z^{\prime \prime}$ where $\pi_{i+1}: Z_{i+1} \rightarrow Z_{i}$ is the blow-up along $\tau_{i}$. First of all, there is a cone $\tau_{j}$ contained in cone $\left(v_{1}, v_{2}, v_{3}, v_{1}+v_{2}-v_{3}\right)$, because otherwise the anticanonical bundle of $Z^{\prime \prime}$ is not ample. We take $j$ minimal. By Lemma 5.1 and by the minimality of $j$, $\tau_{j}$ is cone $\left(v_{1}, v_{2}\right)$. We want to reorder the cones associated to the subvarieties along which we are blowing-up. Clearly this operation is not well defined in general. 
We consider the following sequence of blow-ups: $Z=Z_{0}^{\prime} \leftarrow Z_{1}^{\prime} \leftarrow \cdots \leftarrow Z_{j+1}^{\prime}$, where $\pi_{0}^{\prime}: Z_{1}^{\prime} \rightarrow Z_{0}^{\prime}$ is the blow-up along $\tau_{j}$ and $\pi_{i+1}^{\prime}: Z_{i+1}^{\prime} \rightarrow Z_{i}^{\prime}$ is the blow-up along $\tau_{i-1}$ for each $i \geq 1$. Let $\Delta_{i}^{\prime}=\Delta_{Z_{i}^{\prime}}$. We show that these blow-ups are well defined and $Z_{j+1}^{\prime}=Z_{j+1}$.

The cone $\tau_{i-1}$ belongs to $\Delta_{i}^{\prime}$ for each $1 \leq i \leq j$ because $\tau_{i}$ is contained in $|\Delta| \backslash$ cone $\left(v_{1}, v_{2}, v_{3}, v_{1}+v_{2}-v_{3}\right)$ for each $i \leq j$. Moreover, the elements of $\Delta_{1}^{\prime}(3)$ not contained in cone $\left(v_{1}, v_{2}, v_{3}, v_{1}+v_{2}-v_{3}\right)$ are exactly the elements of $\Delta_{0}(3) \backslash\left\{\operatorname{cone}\left(v_{1}, v_{2}, v_{3}\right), \operatorname{cone}\left(v_{1}, v_{2}, v_{1}+v_{2}-v_{3}\right)\right\}$.

$Z$ is the union of the following two open $S$-subvarieties: $U_{1}$ whose fan has maximal cones cone $\left(v_{1}, v_{2}, v_{3}\right)$ and cone $\left(v_{1}, v_{2}, v_{1}+v_{2}-v_{3}\right)$; and $U_{2}$ whose fan has maximal cones $\Delta(3) \backslash\left\{\operatorname{cone}\left(v_{1}, v_{2}, v_{3}\right)\right.$, cone $\left.\left(v_{1}, v_{2}, v_{1}+v_{2}-v_{3}\right)\right\}$.

The blow-up $\pi_{0}^{\prime}$ induces an isomorphism between $U_{2}$ and its inverse image, because cone $\left(v_{1}, v_{2}\right)$ is not contained in any maximal cone of $U_{2}$. In the same way $\pi_{j}$ induces an isomorphism between the inverse image of $U_{2}$ in $Z_{j}$ and its inverse image in $Z_{j+1}$. So the inverse image of $U_{2}$ in $Z_{j+1}$ is isomorphic to the inverse image of $U_{2}$ in $Z_{j+1}^{\prime}$. Moreover $\pi_{j}^{\prime} \circ \cdots \circ \pi_{2}^{\prime}$ induces an isomorphism between $\left(\pi_{1}^{\prime}\right)^{-1}\left(U_{1}\right)$ and its inverse image. In the same way $\pi_{j-1} \circ \cdots \circ \pi_{1}$ induces an isomorphism between $U_{1}$ and its inverse image. So the inverse image of $U_{1}$ in $Z_{j+1}$ is isomorphic to the inverse image of $U_{1}$ in $Z_{j+1}^{\prime}$. The lemma follows because there is at most one morphism between two toric $S$-varieties extending the identity automorphism of $S$.

We now restrict the possible Fano symmetric varieties with rank three (and fixed $G / H)$ that are obtainable as before to a finite explicit list.

Proposition 5.1. The toric varieties obtainable from $\mathbb{A}^{3}$ by a sequence of blowups and with ample anticanonical bundle are, up to isomorphisms:

1. $\mathbb{A}^{3}$

2. a 2-blow-up of $\mathbb{A}^{3}$;

3. a 3-blow-up of $\mathbb{A}^{3}$;

4. the variety whose fan has maximal cones: cone $\left(e_{1}, e_{1}+e_{2}, e_{1}+e_{2}+e_{3}\right)$, cone $\left(e_{1}, e_{3}, e_{1}+e_{2}+e_{3}\right)$, cone $\left(e_{2}, e_{3}, e_{1}+e_{2}+e_{3}\right)$ and cone $\left(e_{2}, e_{1}+e_{2}, e_{1}+e_{2}+e_{3}\right)$; this variety is obtainable from $\mathbb{A}^{3}$ by two consecutive blow-ups along subvarieties of codimension two;

5. the variety whose fan has maximal cones: cone $\left(e_{1}, e_{3}, e_{1}+e_{2}+2 e_{3}\right)$, cone $\left(e_{1}\right.$, $\left.e_{1}+e_{2}+e_{3}, e_{1}+e_{2}+2 e_{3}\right), \operatorname{cone}\left(e_{1}, e_{2}, e_{1}+e_{2}+e_{3}\right)$, cone $\left(e_{2}, e_{1}+e_{2}+e_{3}, e_{1}+\right.$ $\left.e_{2}+2 e_{3}\right)$ and cone $\left(e_{2}, e_{3}, e_{1}+e_{2}+2 e_{3}\right)$; this variety is obtainable from $\mathbb{A}^{3}$ by a 3-blow up followed by a 2-blow up. 
Proof. Similarly to $\S 4$, we want to find an explicit set $\left\{X_{i}\right\}$ of symmetric varieties such that: 1) they satisfy $(H)$, in particular they are not Fano; 2$)$ there are finitely many symmetric varieties $\left\{Y_{j}\right\}$ that do not dominate any $X_{i}$, but are obtainable from $X_{0}$ through a sequence of blow-ups. In such a case it is sufficient to study the $Y_{j}$; indeed we will study them to find the $X_{i}$.

We proceed as follows: the anticanonical bundle of $\mathbb{A}^{3}$ is ample, so we consider all the possible blow-ups of $\mathbb{A}^{3}$. Let $Z$ be a blow-up of $\left.\mathbb{A}^{3}: 1\right)$ if $Z$ satisfies $(H)$ we know that there is no toric variety with ample anticanonical bundle and obtainable from $Z$ by a sequence of blow-ups; 2) if $Z$ satisfies $(W H)$, we study the variety $Z^{\prime}$ of Lemma $5.3 ; 3$ ) finally, if the anticanonical bundle $Z$ is ample, we reiterate the procedure. Observe that a priori it is possible that $Z$ belongs to none of the previous cases. If two blow-ups of a given variety are isomorphic, we can examine only one of them.

We consider explicitly only some examples to illustrate the strategy. We draw the fans of the varieties we wish to study. In the figure we indicate by $(A)$ that the anticanonical bundle of the variety is ample, and by $(H)$, respectively $(W H)$, that the variety satisfies the hypotheses of Lemma 5.1, resp. 5.3, with respect to $\sigma$ and $\sigma^{\prime}$. In the last two cases we indicate $\sigma \cap \sigma^{\prime}$ with a wavy line. We will use the following notation: $Z_{0}$ is the variety $\mathbb{A}^{3}$ associated with the wonderful compactification; $Z_{1}, Z_{2}, \ldots$ are the blow-ups of $Z_{0}$. Given a variety $Z_{i_{1} \ldots i_{k}}$ we denote its blow-ups by $Z_{i_{1} \ldots i_{k} 1}, \ldots, Z_{i_{1} \ldots i_{k} n}$. Moreover, given a variety $Z_{i_{1}, \ldots, i_{k}}$ which satisfies $(W H)$ we denote by $Z_{i_{1}, \ldots, i_{k} b}$ the variety defined in Lemma 5.3; if $Z_{i_{1}, \ldots, i_{k} b}$ again satisfies $(W H)$ we denote by $Z_{i_{1}, \ldots, i_{k}}$ c the variety defined in Lemma 5.3 and so on. Finally, we denote a vector $a e_{1}+b e_{2}+c e_{3}$ by $(a, b, c)$.

Let $Z_{0}=\mathbb{A}^{3}$; it has ample canonical bundle. Indeed it has trivial Picard group. There are three 2-blow-ups of $Z_{0}$, but they are all isomorphic to the blow-up $Z_{1}$ along cone $\left(e_{1}, e_{2}\right)$. There is a unique 3-blow-up $Z_{2}$ of $Z_{0}$ (along cone $\left(e_{1}, e_{2}, e_{3}\right)$ ).

Let $Z_{11}$ be the blow-up of $Z_{1}$ along cone $\left(e_{1}, e_{3}\right)$; it satisfies $(W H)$ with respect to cone $\left(e_{3}, e_{1}+e_{2}, e_{1}+e_{3}\right)$ and cone $\left(e_{3}, e_{1}+e_{2}, e_{2}\right)$; indeed $\left(e_{1}+e_{3}\right)+e_{2}=$ $e_{3}+\left(e_{1}+e_{2}\right)$ and cone $\left(e_{3}, e_{1}+e_{2}, e_{1}+e_{3}\right) \cap \operatorname{cone}\left(e_{3}, e_{1}+e_{2}, e_{2}\right)=\operatorname{cone}\left(e_{3}, e_{1}+e_{2}\right)$. Hence we have to study the blow-up $Z_{11 b}$ of $Z_{11}$ along cone $\left(e_{3}, e_{1}+e_{2}\right)$.

Let $Z_{12}$ be the blow-up $Z_{1}$ along cone $\left(e_{1}, e_{1}+e_{2}\right)$; it satisfies $(H)$ with respect to cone $\left(e_{3}, e_{1}+e_{2}, 2 e_{1}+e_{2}\right)$ and cone $\left(e_{3}, e_{1}+e_{2}, e_{2}\right)$. Indeed, $\left(2 e_{1}+e_{2}\right)+e_{2}=$ $2\left(e_{1}+e_{2}\right)$ and cone $\left(e_{3}, e_{1}+e_{2}, 2 e_{1}+e_{2}\right) \cap \operatorname{cone}\left(e_{3}, e_{1}+e_{2}, e_{2}\right)=\operatorname{cone}\left(e_{3}, e_{1}+e_{2}\right)$.

The following varieties have not been drawn: 1) the blow-up $Z_{146 b}$ of $Z_{146}$ along cone $\left(e_{1}, e_{1}+e_{2}+e_{3}\right)$, which is $\left.Z_{1 c} ; 2\right)$ the blow-up $Z_{23}$ of $Z_{2}$ along cone $\left(e_{1}, e_{2}\right)$, which is $\left.Z_{14} ; 3\right)$ the blow-up $Z_{229}$ of $Z_{22}$ along cone $\left(e_{1}, e_{2}\right)$, which is $Z_{143}$. 
Now, one can easily prove Theorems $2.3,2.4$ and 2.5 by verifying when the weights associated to the anticanonical bundle of a variety as in the previous lemma are strictly antidominant.
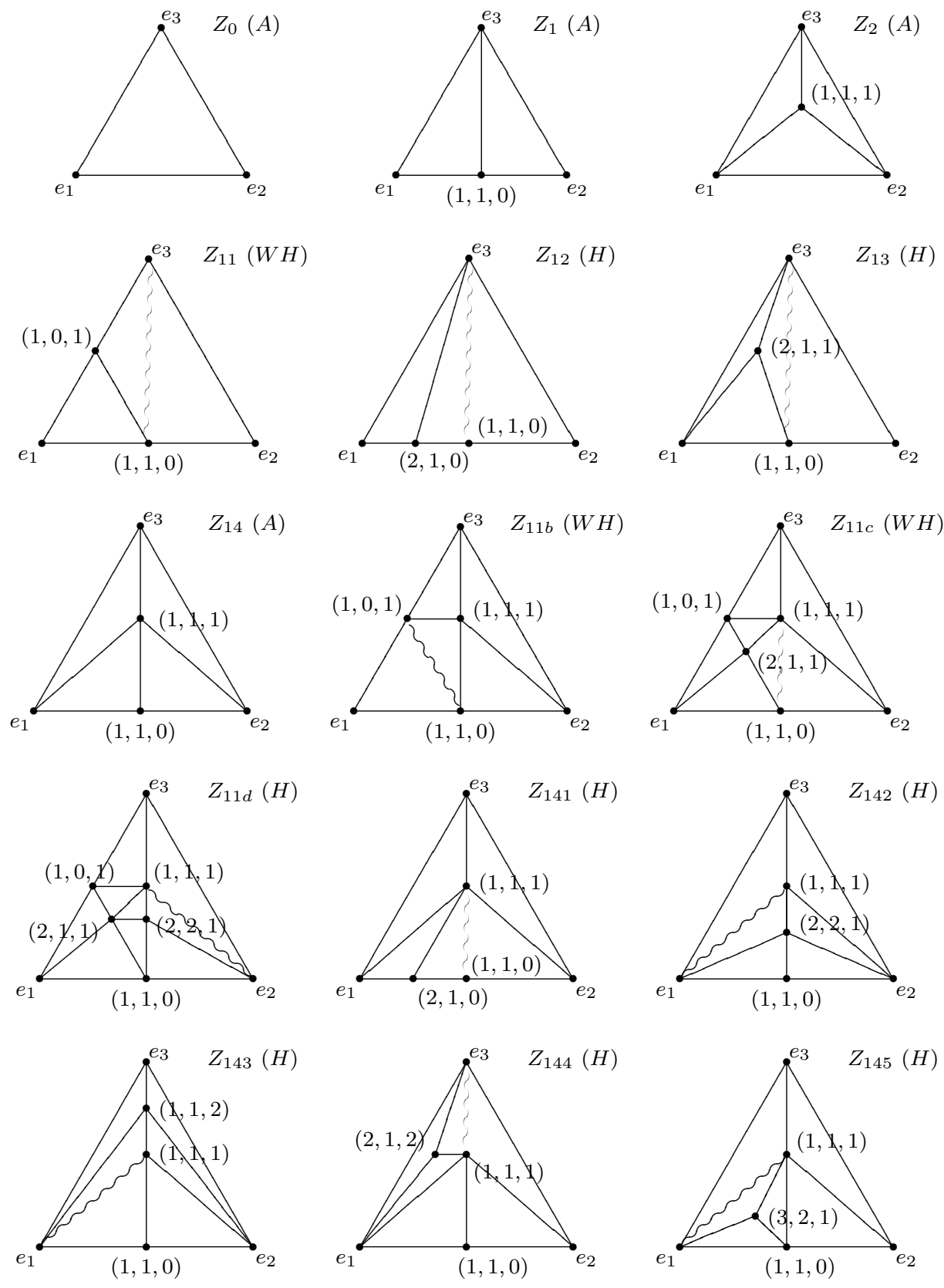

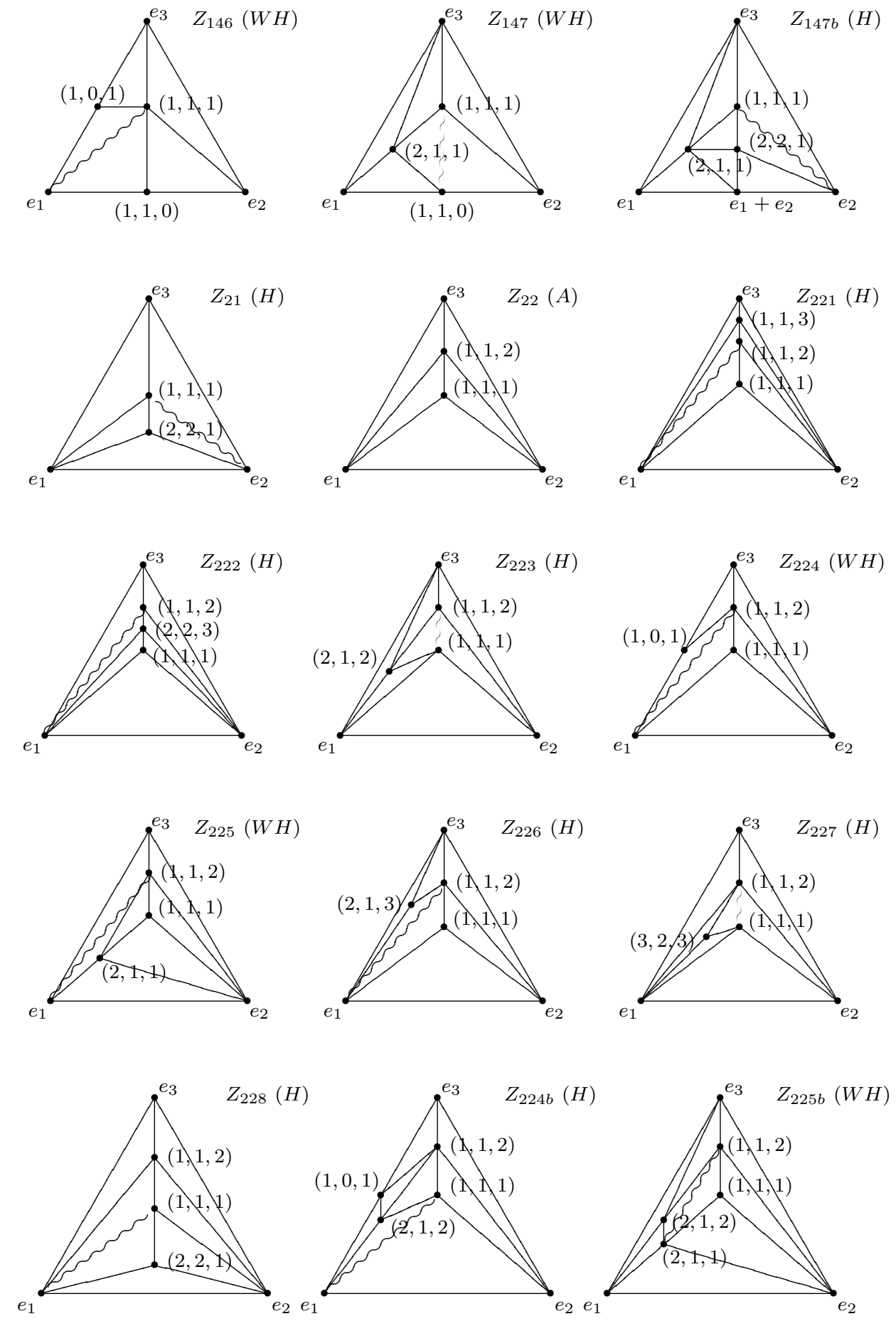


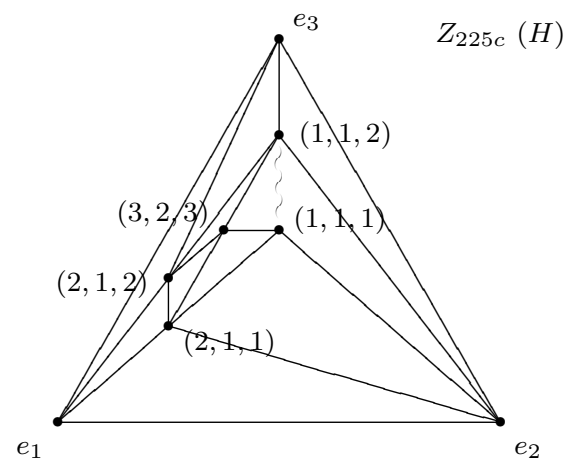

\section{$\S 6$. (Quasi-) Fano symmetric varieties of rank 2}

In this section we consider the quasi-Fano locally factorial symmetric varieties with rank 2 (and $G$ only reductive). We have seen in $\S 2.4$ that if $\rho$ is injective then $X$ is determined by $\Delta_{X}[p]$. To prove the classification of 2-rank Fano symmetric varieties we will consider three cases separately: 1) $X$ is simple; 2) $X$ is toroidal; 3) $X$ is neither simple nor toroidal. In the first case, one can study very easily the ampleness of $-K_{X}$, but to study the smoothness of $X$ is slightly more complicated. In the second case, first we will prove an upper bound on the number of 1-cones of $\Delta_{X}$. In the third case we use the local factoriality of $X$ to prove that $R_{G, \theta}$ is $A_{2}$, $A_{1}, B C_{1}$ or decomposable. The case of quasi-Fano varieties is roughly reduced to the Fano case by Lemma 6.4, which allows one to associate to any quasi-Fano symmetric variety a new quasi-Fano symmetric variety that is "almost always" Fano.

\section{$\S 6.1$. Fano symmetric varieties}

We begin by classifying the Fano varieties with $G$ semisimple. Recall that we have to verify that, given any $(C, F) \in \mathcal{F}(X), k_{C}(v)<1$ for each $v \in N(X) \backslash C$ and $k_{C}(\rho(D))<\left(2 \delta-2 \delta_{0}\right)(\rho(D))$ for each $D \in D(G / H) \backslash F$. One can show that if $X$ is simple then it is quasi- $\mathbb{Q}$-Fano. For example, if $\rho$ is injective, then $\operatorname{Pic}(X)=\mathbb{Z}$ and $K_{Z}$ is not big because $X$ is rational. Moreover, every simple and normal $G$-variety is projective by a classical result of Sumihiro. But in general a simple symmetric variety is not smooth or even locally factorial. Now, we want to find some necessary conditions for the ampleness of $-K_{X}$ when $X$ is non-simple. We consider two cases: (i) $G / H$ is indecomposable, while $X$ is neither simple nor toroidal; (ii) $X$ is toroidal.

Lemma 6.1. Let $X$ be a locally factorial projective symmetric variety. Suppose that $G / H$ is indecomposable and $X$ is neither simple nor toroidal. Then $R_{G, \theta}=A_{2}$, $H=G^{\theta}$ and $\mathcal{F}(X)$ contains $\left(\operatorname{cone}\left(-\alpha_{1}^{\vee}-\alpha_{2}^{\vee}\right), \emptyset\right)$. 
There is a simple restricted coroot $\alpha_{i}^{\vee}$ in $\Delta[p]$ because $X$ is not toroidal. Thus there is a colored cone $\left(\operatorname{cone}\left(\alpha_{i}^{\vee}, v\right), F\right)$ in $\mathcal{F}(X)$, where $v$ is an antidominant and regular weight. Indeed $\operatorname{int}\left(\operatorname{cone}\left(\alpha_{i}^{\vee}, v\right) \cap C^{-} \neq \emptyset\right.$ and $X$ is not simple (so $\left.v \neq-\omega_{i^{c}}^{\vee}\right)$.

Proof. We do a case-by-case analysis.

1) Suppose $R_{G, \theta}=A_{2}$ and $H=N\left(G^{\theta}\right)$. Then, up to reindexing $R_{G, \theta}$, we have cone $\left(\alpha_{1}^{\vee}, v\right) \in \Delta$ with $v:=-\frac{x}{3} \alpha_{1}^{\vee}-\frac{y}{3} \alpha_{2}^{\vee} \in \operatorname{int}\left(C^{-}\right)$primitive. We can write $-\omega_{2}^{\vee}$ as a positive integral combination of $\alpha_{1}^{\vee}$ and $v$; thus $y \in\{1,2\}$. Moreover, $0<\left(-v, \alpha_{2}\right)=-x / 3+2 y / 3$, so $0<x<2 y$. If $y=1$, then $v=-\frac{1}{3}\left(\omega_{1}^{\vee}+\omega_{2}^{\vee}\right) \notin$ $\chi_{*}(S)$. If $y=2$, we have three possibilities: (i) $v=-\omega_{2}^{\vee}$ which is not regular; (ii) $v=-\frac{2}{3}\left(\omega_{1}^{\vee}+\omega_{2}^{\vee}\right) \notin \chi_{*}(S)$; (iii) $v=-\alpha_{1}^{\vee}-\frac{2}{3} \alpha_{2}^{\vee} \notin \chi_{*}(S)$.

2) Suppose $R_{G, \theta}=A_{2}$ and $H=G^{\theta}$. Then, up to reindexing $R_{G, \theta}$, we have $\operatorname{cone}\left(\alpha_{1}^{\vee}, v\right) \in \Delta$ with $v:=-x \alpha_{1}^{\vee}-y \alpha_{2}^{\vee}$ primitive. Hence $y=1$ because $\left\{\alpha_{1}^{\vee}, v\right\}$ is a basis of $\chi_{*}(S)$. Moreover, $0<\left(-v, \alpha_{2}\right)=-x+2$, so $v=-\alpha_{1}^{\vee}-\alpha_{2}^{\vee}$ as in the statement.

3) Suppose $R_{G, \theta}=B_{2}$ and cone $\left(\alpha_{1}^{\vee}, v\right) \in \Delta$, with $v:=-x \alpha_{1}^{\vee}-\frac{y}{2} \alpha_{2}^{\vee}$ primitive. We can write $-\omega_{2}^{\vee}=-\alpha_{1}^{\vee}-\alpha_{2}^{\vee}$ as a positive integral combination of $\alpha_{1}^{\vee}$ and $v$; thus $y \in\{1,2\}$. Moreover, $0<\left(-v, \alpha_{2}\right)=-x+y$, so $0<x<y$. Therefore $v=-\omega_{2}^{\vee}$ is not regular.

4) Suppose $R_{G, \theta}=B_{2}$ and $\operatorname{cone}\left(\alpha_{2}^{\vee}, v\right) \in \Delta$. If $H=N\left(G^{\theta}\right)$, then $\left\{\alpha_{2}^{\vee}, v\right\}$ cannot be a basis of $\chi_{*}(S)=\mathbb{Z} \alpha_{1}^{\vee} \oplus \mathbb{Z} \frac{\alpha_{2}^{\vee}}{2}$.

5) Suppose $R_{G, \theta}=B_{2}, H=G^{\theta}$, and cone $\left(\alpha_{2}^{\vee}, v\right) \in \Delta$ with $v:=-x \alpha_{1}^{\vee}-$ $y \alpha_{2}^{\vee}$ primitive. Then $x=1$ because $\left\{\alpha_{2}^{\vee}, v\right\}$ is a basis of $\chi_{*}(S)$. Moreover, $0<$ $\left(-v, \alpha_{1}\right)=2-2 y$, so $0<y<1$.

6) Suppose $R_{G, \theta}=B C_{2}$ and $\operatorname{cone}\left(\alpha_{1}^{\vee}, v\right) \in \Delta$ with $v:=-x \alpha_{1}^{\vee}-y \alpha_{2}^{\vee}$ primitive. As before $y=1$. Moreover, $0<\left(-v, \alpha_{2}\right)=-x+1$, so $0<x<1$.

7) Suppose $R_{G, \theta}=B C_{2}$ and $\operatorname{cone}\left(\alpha_{2}^{\vee}, v\right) \in \Delta$ with $v:=-x \alpha_{1}^{\vee}-y \alpha_{2}^{\vee}$ primitive. As before $x=1$ and $0<\left(-v, \alpha_{1}\right)=2-y$, so $v=-\omega_{1}^{\vee}$ is not regular.

8) Suppose $R_{G, \theta}=G_{2}$ and $\operatorname{cone}\left(\alpha_{1}^{\vee}, v\right) \in \Delta$ with $v:=-x \alpha_{1}^{\vee}-y \alpha_{2}^{\vee}$ primitive. As before $y=1$ and $0<\left(-v, \alpha_{2}\right) \leq-3 x+2$, so $0<x<2 / 3$.

9) Suppose $R_{G, \theta}=G_{2}$ and cone $\left(\alpha_{2}^{\vee}, v\right) \in \Delta$ with $v:=-x \alpha_{1}^{\vee}-y \alpha_{2}^{\vee}$ primitive. As before $x=1$. Moreover, $0<\left(-v, \alpha_{1}\right)=2-y$ and $0<\left(-v, \alpha_{2}\right)=-3+2 y$; thus $3<2 y<4$.

In the next two lemmas, we do not make any hypothesis on the regularity of $X$.

Lemma 6.2. Let $X$ be a Fano symmetric variety of rank 2 with $G$ semisimple. Then $\mathcal{F}(X)$ contains at most three colored 1-cones. 
Proof. Suppose that $\mathcal{F}(X)$ contains $\left(\sigma_{i}:=\operatorname{cone}\left(v_{i}, v_{i+1}\right), \emptyset\right)$ with $i=1,2,3$ and $v_{1}, v_{2}, v_{3}, v_{4}$ primitive. Recall that all the 1-dimensional cones are contained in $C^{-}$, so they do not have colors. We can write $v_{2}$ as a positive linear combination $x v_{1}+y v_{4}$ of $v_{1}$ and $v_{4}$. Then $k_{\sigma_{2}}\left(v_{1}\right)<k\left(v_{1}\right)=1, k_{\sigma_{2}}\left(v_{4}\right)<k\left(v_{4}\right)=1$ and $1=k_{\sigma_{2}}\left(v_{2}\right)=x k_{\sigma_{2}}\left(v_{1}\right)+y k_{\sigma_{2}}\left(v_{4}\right) \leq 0$, which gives a contradiction.

Lemma 6.3. Let $X$ be a Fano non-simple toroidal symmetric variety. Then $X_{0}$ is smooth and $\Delta[p]$ is either $\left\{e_{1}, e_{1}+r e_{2}, e_{2}\right\}$ or $\left\{e_{1}, r e_{1}+e_{2}, e_{2}\right\}$. These varieties are smooth if and only if $r=1$.

Proof. By the previous lemma, we have $\Delta[p]=\left\{e_{1}, e_{2}, v\right\}$ for an appropriate $v$. First suppose that $X_{0}$ is smooth, i.e. $\chi_{*}(S)=\mathbb{Z} e_{1} \oplus \mathbb{Z} e_{2}$, and write $v=x_{1} e_{1}+x_{2} e_{2}$. For each $i$, let $\sigma_{i}=\operatorname{cone}\left(e_{i}, v\right)$ and $\left\{i, i^{c}\right\}=\{1,2\}$, so $k_{\sigma_{i}}$ is $e_{i}^{*}+\frac{1-x_{i}}{x_{i^{c}}} e_{i^{c}}^{*}$. If $x_{1} \geq 2$ and $x_{2} \geq 2$, then $\frac{1-x_{1}}{x_{2}}$ and $\frac{1-x_{2}}{x_{1}}$ are strictly negative integers, so $x_{1} \geq x_{2}+1 \geq$ $x_{1}+2$, a contradiction.

Suppose now that $X_{0}$ is singular. Then $R_{G, \theta}$ is either $A_{2}$ or $A_{1} \times A_{1}$. In the first case, $e_{i}=-3 \omega_{i}^{\vee}$ and the strictly positive integer $k\left(-\omega_{1}^{\vee}-\omega_{2}^{\vee}\right)$ is smaller than $\frac{1}{3} k\left(-3 \omega_{1}^{\vee}\right)+\frac{1}{3} k\left(-3 \omega_{2}^{\vee}\right)=\frac{2}{3}$, a contradiction. Finally suppose $R_{G, \theta}=A_{1} \times A_{1}$ and $\chi_{*}(S)=\mathbb{Z} 2 \omega_{2}^{\vee} \oplus \mathbb{Z}\left(\omega_{1}^{\vee}+\omega_{2}^{\vee}\right)$. Then there is $i$ such that $-\omega_{1}^{\vee}-\omega_{2}^{\vee}=a v+b\left(-2 \omega_{i}^{\vee}\right)$ with $a, b \geq 0$. The integer $a+b=k\left(-\omega_{1}-\omega_{2}\right)$ is strictly less than $\frac{1}{2} k\left(-2 \omega_{1}\right)+$ $\frac{1}{2} k\left(-2 \omega_{2}\right)=1$, a contradiction.

Note that the previous two lemmas apply also to a toroidal symmetric variety with $-K_{X}$ ample, $|\mathcal{F}(X)|$ convex and generated by a basis of $\chi_{*}(S)$ (without supposing $X$ is compact). Using Lemma 6.3, one can easily prove Proposition 2.1. Now, we prove Theorem 2.6 (and Theorem C).

Proof of Theorem 2.6. Recall that an element $v \in \Delta[p]$ is a simple coroot if it is the image of a color by $\rho$, while it is an antidominant weight if it is the image of a $G$-stable divisor (in this case $v \in N(X)$ ). The first item of the theorem holds because $-K_{X}$ is linearly equivalent to a $G$-stable divisor. Indeed suppose that there are $D_{1} \in D(X)$ and $D_{2} \in D(G / H)$ such that $\rho\left(D_{1}\right)=\rho\left(D_{2}\right)$; then the coefficients $a_{D_{1}}$ and $a_{D_{2}}$ of $-K_{X}$ with respect to $D_{1}$ and $D_{2}$ are equal. Let $h_{X}$ be the piecewise linear function associated to $-K_{X}$. If $-K_{X}$ is ample and $D_{2} \notin D(X)$, then $a_{D_{1}}=h_{X}\left(\rho\left(D_{1}\right)<h_{X}\left(\rho\left(D_{2}\right)=a_{D_{2}}\right.\right.$, which gives a contradiction. Finally, observe that if $X$ is locally factorial, then $\rho$ is injective over $D(X)$.

Let $a_{i}:=\left(2 \delta-2 \delta_{0}\right)\left(\alpha_{i}^{\vee}\right)$ for each $i$; recall that $a_{1}, a_{2} \geq 1$ (see $\left.\S 3\right)$. In the following we always suppose $\sharp \rho(D(X))=\sharp \rho^{-1}(\rho(D(X)))$.

(I) Suppose $X$ simple. If $X$ is toroidal (i.e. $X=X_{0}$ ) we have to exclude the following cases by $\S 3$ : (i) $G / H=G_{2} /\left(S L_{2} \times S L_{2}\right)$; (ii) $R_{G, \theta}=A_{2}$ and $H=G^{\theta}$; 
(iii) $R_{G, \theta}=A_{1} \times A_{1}$ and $\chi_{*}(S)=\mathbb{Z} 2 \omega_{1}^{\vee} \oplus \mathbb{Z}\left(\omega_{1}^{\vee}+\omega_{2}^{\vee}\right)$. In the first case $X_{0}$ is not Fano, while in the last two cases $X_{0}$ is not smooth. If $\rho(D(X))=\left\{\alpha_{1}^{\vee}\right\}$, then $\theta$ is indecomposable because $C(X)$ is strictly convex. Moreover, $\Delta[p]=\left\{\alpha_{1}^{\vee}, e_{1}=\right.$ $\left.-x_{1} \alpha_{1}^{\vee}-x_{2} \alpha_{2}^{\vee}\right\}$ and $k_{X}=a_{1} \omega_{1}-\frac{x_{1} a_{1}+1}{x_{2}} \omega_{2}$; so $k_{X}\left(\alpha_{2}^{\vee}\right) \leq 0<a_{2}$. Thus we have only to verify that $X$ is locally factorial. We have to exclude two cases: 1$)$ $\Delta(2)=\left\{\operatorname{cone}\left(\alpha_{1}^{\vee},-\omega_{1}^{\vee}\right)\right\}$ and $\left.R_{G, \theta}=G_{2} ; 2\right) \Delta(2)=\left\{\operatorname{cone}\left(\alpha_{2}^{\vee},-\omega_{2}^{\vee}\right)\right\}, R_{G, \theta}=B_{2}$ and $H=N\left(G^{\theta}\right)$.

(II) Suppose now that $X$ is not simple. In the toroidal case we have $\Delta[p]=$ $\left\{e_{1}, e_{2}, e_{1}+e_{2}\right\}$ and $X_{0}$ is smooth by Lemma 6.3 . This variety is Fano if and only if $-2 \delta+2 \delta_{0}+e_{i}^{*}$ is antidominant and regular for each $i$.

(III) Assume moreover that $X$ is not toroidal. Suppose first $\theta$ is indecomposable. Then, by Lemma $6.1, R_{G, \theta}=A_{2}, H=G^{\theta}$ and $\Delta(2)$ contains cone $\left(\alpha_{1}^{\vee},-\alpha_{1}^{\vee}-\alpha_{2}^{\vee}\right)$, up to reindexing. If $\rho(D(X))$ also contains $\alpha_{2}^{\vee}$ then $\Delta(2)=$ $\left\{\operatorname{cone}\left(\alpha_{1}^{\vee},-\alpha_{1}^{\vee}-\alpha_{2}^{\vee}\right)\right.$, cone $\left.\left(\alpha_{2}^{\vee},-\alpha_{1}^{\vee}-\alpha_{2}^{\vee}\right)\right\}$. Moreover, $\widetilde{k}_{X}\left(\alpha_{1}^{\vee}\right)=\widetilde{k}_{X}\left(\alpha_{2}^{\vee}\right)=0$ and $\widetilde{k}_{X}\left(-\alpha_{1}^{\vee}-\alpha_{2}^{\vee}\right) \geq 1$, so $\widetilde{k}_{X}$ is strictly convex.

If $\rho(D(X))=\left\{\alpha_{1}^{\vee}\right\}$, then $\Delta[p]$ contains $-3 \omega_{1}^{\vee}$. Note that $\left\{-\alpha_{1}^{\vee}-\alpha_{2}^{\vee},-3 \omega_{1}^{\vee}\right\}$ is a basis of $\chi_{*}(S)$. By Lemma 6.3 we have two possibilities for $\Delta[p]:\left\{\alpha_{1}^{\vee},-\alpha_{1}^{\vee}-\alpha_{2}^{\vee}\right.$, $\left.-3 \omega_{1}^{\vee}\right\}$ or $\left\{\alpha_{1}^{\vee},-\alpha_{1}^{\vee}-\alpha_{2}^{\vee},-4 \omega_{1}^{\vee}-\omega_{2}^{\vee},-3 \omega_{1}^{\vee}\right\}$. In the first case there is no condition because the weights of $k$ are $a_{1} \omega_{1}-\left(a_{1}+1\right) \omega_{2}$ and $-\omega_{2}$. In the last case we have to impose that $a_{1}, a_{2}>1$. Indeed the weights of $k$ are $a_{1} \omega_{1}-\left(a_{1}+1\right) \omega_{2},-\alpha_{2}$ and $-\omega_{1}+\omega_{2}$. Moreover $\left(-\alpha_{2}\right)\left(\alpha_{1}^{\vee}\right)<a_{1}$ and $\left(-\omega_{1}+\omega_{2}\right)\left(\alpha_{2}^{\vee}\right)<a_{2}$. In $\S 3$, we have seen that $a_{1} \leq 1, a_{2} \leq 1$ if and only if $\theta=-$ id.

(IV) Suppose now $\theta$ is decomposable. Note that $\sharp D(G / H)=2$ if $\chi_{*}(S)=$ $\mathbb{Z}\left(\omega_{1}^{\vee}+\omega_{2}^{\vee}\right) \oplus \mathbb{Z} 2 \omega_{2}^{\vee}$, because any $n \in H \backslash G^{\theta}$ exchanges the colors of $G / G^{\theta}$ associated to the same coroot. Thus in this case any projective symmetric variety is $\mathbb{Q}$-factorial.

First suppose $D(X)=D(G / H)$. Then $\Delta(2)$ contains cone $\left(\alpha_{1}^{\vee}, x\left(-m \alpha_{1}^{\vee}-\alpha_{2}^{\vee}\right)\right)$ and cone $\left(\alpha_{2}^{\vee}, x\left(-\alpha_{1}^{\vee}-r \alpha_{2}^{\vee}\right)\right)$ with $x \in\{1,1 / 2\}$. Note that $\mathbb{R}^{+}\left(-m \alpha_{1}^{\vee}-\alpha_{2}^{\vee}\right)=$ $\mathbb{R}^{+}\left(-\alpha_{1}^{\vee}-\frac{1}{m} \alpha_{2}^{\vee}\right)$ and $x\left(-\alpha_{1}^{\vee}-r \alpha_{2}^{\vee}\right) \notin \operatorname{cone}\left(\alpha_{1}^{\vee}, x\left(-m \alpha_{1}^{\vee}-\alpha_{2}^{\vee}\right)\right)$ so $r \leq 1 / m$. Therefore $\Delta(2)=\left\{\operatorname{cone}\left(\alpha_{1}^{\vee}, x\left(-\alpha_{1}^{\vee}-\alpha_{2}^{\vee}\right)\right)\right.$, cone $\left.\left(\alpha_{2}^{\vee}, x\left(-\alpha_{1}^{\vee}-\alpha_{2}^{\vee}\right)\right)\right\}$.

(V) Suppose now that $\rho(D(X))$ contains exactly one coroot, say $\alpha_{1}^{\vee}$. Suppose $X_{0}$ is smooth and let $\sigma_{0}:=\operatorname{cone}\left(\alpha_{1}^{\vee},-m \alpha_{1}^{\vee}+e_{2}\right)$ be in $\Delta(2)$. The case of $X_{0}$ singular is very similar. We can apply Lemma 6.3 to the maximal open toroidal subvariety $X^{\prime}$ of $X$ (whose colored fan has support cone $\left(-\alpha_{1}^{\vee},-m \alpha_{1}^{\vee}+e_{2}\right)$ ). There are two possibilities for $\Delta_{X^{\prime}}$ : its maximal cones are either $\left\{\sigma_{1}:=\operatorname{cone}\left(-\alpha_{1}^{\vee},-m \alpha_{1}^{\vee}+e_{2}\right)\right\}$ or $\left\{\sigma_{1}:=\operatorname{cone}\left(-m \alpha_{1}^{\vee}+e_{2},-(m+1) \alpha_{1}^{\vee}+e_{2}\right), \sigma_{2}:=\operatorname{cone}\left(-\alpha_{1}^{\vee},-(m+1) \alpha_{1}^{\vee}+e_{2}\right)\right\}$. In the first case the unique non-trivial condition is $k_{\sigma_{1}}\left(\alpha_{2}^{\vee}\right)<a_{2}$, which is equivalent to $\widetilde{k}_{\sigma_{1}}\left(\alpha_{2}^{\vee}\right)=\left(\psi_{2}(m-1)\right)\left(\alpha_{2}^{\vee}\right)<0$. In the second case the unique non-trivial condition is $\widetilde{k}_{\sigma_{2}}\left(\alpha_{2}^{\vee}\right)=\left(\psi_{2}(m)\right)\left(\alpha_{2}^{\vee}\right)<0$. 
Now, we are interested in which of these varieties are smooth. First, we explain the conditions for a projective locally factorial symmetric variety $X$ with rank two to be smooth (see [Ru07, Theorems 2.1 and 2.2]). Let $Y$ be an open simple $G$ subvariety of $X$ whose closed orbit is compact; then the associated colored cone $(C, F)$ is 2-dimensional. Write $C=\operatorname{cone}\left(v_{1}, v_{2}\right)$ and let $C^{\vee}=\operatorname{cone}\left(v_{1}^{*}, v_{2}^{*}\right)$ be the dual cone; we take $v_{1}$ and $v_{2}$ primitive. If $X$ is smooth then $\rho$ is injective over $F$ and $\rho(F)$ does not contain any exceptional root. Suppose the latter conditions are satisfied and let $R^{\prime}$ be the root subsystem of $R_{G, \theta}$ generated by the simple roots $\alpha$ such that $\alpha^{\vee} \in \rho(F)$ and $\sharp \rho^{-1}\left(\alpha^{\vee}\right)=1$. If there is no such root, then $Y$ is smooth. Otherwise it is smooth if and only if: (i) $R^{\prime}$ has type $A_{1}$; (ii) up to reindexing, $\frac{1}{2}\left(2 v_{1}^{*}-v_{2}^{*}\right)$ is the fundamental weight of $R^{\prime}$.

Suppose now that $C=\operatorname{cone}\left(\alpha_{1}^{\vee},-r \alpha_{1}^{\vee}-\alpha_{2}^{\vee}\right)$ with $-r \alpha_{1}^{\vee}-\alpha_{2}^{\vee}$ primitive. If $X$ is Fano, then $\sharp \rho^{-1}\left(\alpha_{1}^{\vee}\right)=1$. If moreover $\theta$ is indecomposable, then $R_{G, \theta}=A_{2}$, $H=G^{\theta}$ and $r=1$. Furthermore $R^{\prime}$ is $A_{1}$ and $C^{\vee}=\operatorname{cone}\left(v_{1}^{*}=\omega_{1}-\omega_{2}, v_{2}^{*}=-\omega_{2}\right)$. Hence $\frac{1}{2}\left(2 v_{1}^{*}-v_{2}^{*}\right)=\frac{1}{2} \alpha_{1}$; thus $Y$ is smooth. If $G / G^{\theta}=G_{1} / G_{1}^{\theta} \times G_{2} / G_{2}^{\theta}$, then $R^{\prime}=R_{G_{1}, \theta}, H=G_{1}^{\theta} \times\left(H \cap G_{2}\right)$ and $C^{\vee}=\operatorname{cone}\left(v_{1}^{*}=\omega_{1}-r \omega_{2}, v_{2}^{*}=-\omega_{2}\right)$. Hence $R_{G_{1}, \theta}$ has to be $A_{1}$ and $\frac{1}{2}\left(2 v_{1}^{*}-v_{2}^{*}\right)=\omega_{1}+\left(\frac{1}{2}-r\right) \omega_{2}$ has to be $\frac{1}{2} \alpha_{1}$. Thus $Y$ is not smooth. Suppose now that $R_{G, \theta}=A_{1} \times A_{1}, \chi_{*}(S)=\mathbb{Z} 2 \omega_{1}^{\vee} \oplus \mathbb{Z}\left(\omega_{1}^{\vee}+\omega_{2}^{\vee}\right)$ and $C=\operatorname{cone}\left(\alpha_{1}^{\vee},-\frac{2 r+1}{2} \alpha_{1}^{\vee}-\frac{1}{2} \alpha_{2}^{\vee}\right)$. Then $R^{\prime}$ is $A_{1}$ and we have $C^{\vee}=\operatorname{cone}\left(v_{1}^{*}=\right.$ $\left.\omega_{1}-(2 r+1) \omega_{2}, v_{2}^{*}=-2 \omega_{2}\right)$; moreover, $\frac{1}{2}\left(2 v_{1}^{*}-v_{2}^{*}\right)=\omega_{1}-2 r \omega_{2}$; thus $Y$ is smooth if and only if $r=0$. The other cases are similar.

\section{$\S 6.2$. Smooth quasi-Fano varieties}

Now, we prove Theorem 2.7 (and Theorem D) about the quasi-Fano symmetric varieties. Recall that we have to verify that, given any $(C, F) \in \mathcal{F}(X), k_{C}(v) \leq 1$ for each $v \in N(X)$ and $k_{C}\left(\alpha^{\vee}\right) \leq\left(2 \delta-2 \delta_{0}\right)\left(\alpha^{\vee}\right)$ for each simple restricted coroot $\alpha^{\vee}$. The idea is to use Theorem 2.6 and the following lemma. In the case where $\theta$ is indecomposable, if $-K_{X}$ is nef then it is also big (see $§ 1.8$ ).

Lemma 6.4. Let $X$ be a projective symmetric variety with $-K_{X}$ nef. Then there is a symmetric variety $X^{\prime}$ below $X$ such that the piecewise linear function associated to $-K_{X^{\prime}}$ is strictly convex (over the colored fan of $X^{\prime}$ ) and coincides with the function associated to $-K_{X}$. If $X$ is toroidal, we can choose $X^{\prime}$ toroidal.

Proof. Let $\Delta^{\prime}$ be the fan whose maximal cones are the maximal cones over which $k_{X}$ is linear. For any cone $C \in \Delta^{\prime}$, define $F_{C}$ as $\{D \in D(G / H)$ :

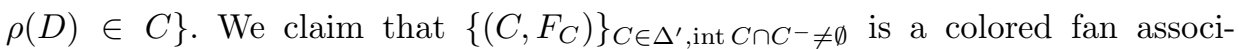
ated to a symmetric variety that satisfies the conditions of the lemma. Note that $\left|\Delta^{\prime}\right|=|\mathcal{F}(X)|$. We have only to prove that the maximal cones of $\Delta^{\prime}$ are strictly convex. Let $C=\operatorname{cone}\left(\alpha_{1}^{\vee}, \ldots, \alpha_{r}^{\vee},-\varpi_{1}^{\vee}, \ldots,-\varpi_{s}^{\vee}\right) \in \Delta^{\prime}(l)\left(\right.$ with $\varpi_{1}^{\vee}, \ldots, \varpi_{s}^{\vee}$ 
dominant) and suppose for contradiction that it contains the line generated by $v=\sum_{i=1}^{r} a_{i} \alpha_{i}^{\vee}+\sum_{j=1}^{s} b_{j}\left(-\varpi_{j}^{\vee}\right)$, where $a_{i}, b_{j} \geq 0$. Then $C$ also contains $\mathbb{R} v^{\prime}$, where $v^{\prime}=\sum b_{j}\left(-\varpi_{j}^{\vee}\right)$. Indeed $\alpha_{1}^{\vee}, \ldots, \alpha_{r}^{\vee},-\varpi_{1}^{\vee}, \ldots,-\varpi_{s}^{\vee},-v$ belong to $C$ and $-v^{\prime}=-v+\sum_{i=1}^{r} a_{i} \alpha_{i}^{\vee}$; thus $v^{\prime}$ and $-v^{\prime}$ belong to $C$. Write $-v^{\prime}=\sum_{\alpha \in \bar{R}_{G, \theta}} c_{\alpha} \alpha^{\vee}$; then $C$ contains all the $\alpha^{\vee}$ such that $c_{\alpha} \neq 0$, because $-v^{\prime} \in C \cap C^{+}$and any spherical weight is a positive rational combination of simple restricted roots. Thus, if $v^{\prime} \neq 0$, then $k_{C}\left(-v^{\prime}\right)=\sum c_{\alpha} k_{C}\left(\alpha^{\vee}\right) \geq 0$, while $k_{C}\left(v^{\prime}\right)=\sum b_{j} k_{C}\left(-\varpi_{j}^{\vee}\right)>0$. Suppose now that $v=\sum_{i=1}^{r} a_{i} \alpha_{i}^{\vee}$ with $a_{j} \neq 0$. Then $C$ contains $\mathbb{R} \alpha_{j}^{\vee}$. Write $-\alpha_{j}^{\vee}=\sum_{i=1}^{r} a_{i}^{\prime} \alpha_{i}^{\vee}+\sum_{i=1}^{s} b_{i}^{\prime}\left(-\varpi_{i}^{\vee}\right)$ with positive coefficients. Then there is $j_{0}$ such that $b_{j_{0}} \neq 0$. So $k_{C}\left(-\alpha_{j}^{\vee}\right) \geq b_{j_{0}}>0$ and $k_{C}\left(\alpha_{j}^{\vee}\right)>0$, a contradiction.

Proof of Theorem 2.7. The first property holds because the inequalities in the conditions for the nefness of a Cartier divisor are not strict; moreover given two colors in the inverse image of a simple coroot, the coefficients of $-K_{X}$ with respect to these divisors are equal.

(I) First suppose $\rho$ non-injective. We have to consider the varieties whose fan is as in Theorem 2.6, but that are not Fano because $\sharp \rho^{-1}(\rho(D(X)) \neq \sharp \rho(D(X))$. In such cases $\rho$ has to be injective over $D(X)$, so that $X$ is locally factorial. If $\theta$ is indecomposable we have the following possibilities: (i) $R_{G, \theta}=B C_{2}$ and $\Delta(2)=\left\{\operatorname{cone}\left(\alpha_{2}^{\vee},-\omega_{2}\right)\right\}$; (ii) $R_{G, \theta}=B_{2}, H=G^{\theta}$ and $\Delta(2)=\left\{\operatorname{cone}\left(\alpha_{1}^{\vee},-2 \omega_{1}\right)\right\}$. If $\theta$ is decomposable, we have to consider all the possibilities listed in Theorem 2.6 that correspond to non-toroidal varieties with $\chi_{*}(S)=\mathbb{Z} e_{1} \oplus \mathbb{Z} e_{2}$. We also have to replace the conditions of type $\psi_{i}(m)<0$ by the corresponding conditions $\psi_{i}(m) \leq 0$.

(II) Suppose $X$ toroidal and let $X^{\prime}$ be as in Lemma 6.4. If $X^{\prime}=X$ and is simple, then $G / H=G_{2} /\left(S L_{2} \times S L_{2}\right)$. If $X^{\prime} \neq X$ and is simple, then it must be singular. Otherwise, given any $w=x_{1} e_{1}+x_{2} e_{2} \in \Delta_{X}[p] \backslash \Delta_{X^{\prime}}[p]$, we have $x_{i} \in \mathbb{Z}^{>0}$ and $x_{1}+x_{2}=k(w)=1$. If $R_{G, \theta}=A_{2}$ and $H=G^{\theta}$, then $k_{X}=$ $-\frac{1}{3}\left(\omega_{1}+\omega_{2}\right) \notin \chi(S)$, so $X$ is not locally factorial. Thus $R_{G, \theta}=A_{1} \times A_{1}$ and $\chi_{*}(S)=\mathbb{Z} 2 \omega_{1}^{\vee} \oplus \mathbb{Z}\left(\omega_{1}^{\vee}+\omega_{2}^{\vee}\right)$. Let $w=-x_{1} \alpha_{1}^{\vee}-x_{2} \alpha_{2}^{\vee}$ be in $\Delta_{X}[p] \backslash \Delta_{X^{\prime}}[p] ;$ then $2 x_{1}, 2 x_{2} \in \mathbb{Z}^{>0}$ and $x_{1}+x_{2}=k(w)$ is 1 . Thus $\Delta_{X}[p]=\left\{-\alpha_{1}^{\vee},-\alpha_{2}^{\vee},-\frac{1}{2} \alpha_{1}^{\vee}-\frac{1}{2} \alpha_{2}^{\vee}\right\}$. In this case $-K_{X}$ is nef but non-ample.

If $X^{\prime}$ is not simple, then the standard compactification of $G / H$ has to be smooth because of Lemma 6.3. So it is sufficient to prove the following lemma.

Lemma 6.5. The smooth toric varieties birationally proper over $\mathbb{A}^{2}$ with nef anticanonical divisor are, up to isomorphisms, $\mathbb{A}^{2}$ and $Z_{m}$, where $Z_{m}$ is the variety whose fan has maximal cones $\left\{\operatorname{cone}\left(e_{1}, e_{1}+e_{2}\right)\right.$, cone $\left(e_{1}+e_{2}, e_{1}+2 e_{2}\right), \ldots$, cone $\left(e_{1}+\right.$ $\left.(m-1) e_{2}, e_{1}+m e_{2}\right)$, cone $\left.\left(e_{1}+m e_{2}, e_{2}\right)\right\}$. 
Proof of Lemma 6.5. The piecewise linear function $k_{m}$ associated to the anticanonical bundle of $Z_{m}$ is linear on cone $\left(e_{1}, e_{1}+m e_{2}\right)$. It is easy to see that this function is convex.

Now we show that, given any $Z$ as in the hypotheses, it is isomorphic to $Z_{m}$ for an appropriate $m$. Notice that any smooth toric variety birationally proper over $\mathbb{A}^{2}$ is obtained by a sequence of blow-ups. Thus there is nothing to prove if $\sharp \Delta[p]<4$. Suppose now that $\sharp \Delta[p] \geq 4$; we claim that up to isomorphisms, $\Delta$ contains cone $\left(e_{1}, e_{1}+e_{2}\right)$ and cone $\left(e_{1}+e_{2}, e_{1}+2 e_{2}\right)$. We know that $\tau=\mathbb{R}^{\geq 0}\left(e_{1}+e_{2}\right)$ is contained in $\Delta$.

First of all, we determine the restrictions of $k$ to the cones containing $\tau$, and afterwards we will determine the cones themselves. Let $\sigma=\operatorname{cone}\left(e_{1}+e_{2}, b_{1} e_{1}+\right.$ $\left.b_{2} e_{2}\right) \in \Delta(2)$ be a maximal cone containing $\tau$ and write $k_{\sigma}=x_{1} e_{1}^{*}+x_{2} e_{2}^{*}$, so $k_{\sigma}\left(e_{1}+e_{2}\right)=x_{1}+x_{2}=1$ and $x_{i}=k_{\sigma}\left(e_{i}\right) \leq 1$ for each $i$. Hence $k_{\sigma}$ is $e_{i}^{*}$ for an appropriate $i$ and $b_{i}=k_{\sigma}\left(b_{1} e_{1}+b_{2} e_{2}\right)=1$. Because of the non-singularity of $Z$ the only possibilities for $\sigma$ are cone $\left(e_{1}+e_{2}, e_{1}\right)$, cone $\left(e_{1}+e_{2}, e_{2}\right)$, cone $\left(e_{1}+e_{2}, e_{1}+2 e_{2}\right)$ and cone $\left(e_{1}+e_{2}, 2 e_{1}+e_{2}\right)$. The fan $\Delta$ does not contain both cone $\left(e_{1}+e_{2}, e_{1}+2 e_{2}\right)$ and cone $\left(e_{1}+e_{2}, 2 e_{1}+e_{2}\right)$; otherwise $k_{\text {cone }\left(e_{1}+e_{2}, e_{1}+2 e_{2}\right)}\left(2 e_{1}+e_{2}\right)=2>1=$ $k\left(2 e_{1}+e_{2}\right)$. Observe that if $\Delta$ contains cone $\left(e_{1}+e_{2}, 2 e_{1}+e_{2}\right)$, then $Z$ is isomorphic to a variety whose fan contains cone $\left(e_{1}+e_{2}, e_{1}+2 e_{2}\right)$ by the isomorphism induced by the automorphism of $\chi_{*}(S)$ that exchanges $e_{1}$ and $e_{2}$. So the claim is proved.

Because of the non-singularity of $Z, \Delta$ contains a cone $\sigma=\operatorname{cone}\left(e_{1}+m e_{2}, e_{2}\right)$ for a suitable integer $m$; we want to show that $Z$ is $Z_{m}$. Let $Z^{\prime}$ be the open toric subvariety of $Z$ whose fan $\Delta^{\prime}$ is $\Delta \backslash\left\{\operatorname{cone}\left(e_{1}+m e_{2}, e_{2}\right)\right.$, cone $\left.\left(e_{2}\right)\right\}$.

We claim that, for each integer $r>1$, there is a unique variety $\widetilde{Z}_{r}^{\prime}$ with the following two properties: 1$)$ the fan $\widetilde{\Delta}_{r}^{\prime}$ of $\widetilde{Z}_{r}^{\prime}$ has support cone $\left(e_{1}, e_{1}+r e_{2}\right)$; 2) $\widetilde{Z}_{r}^{\prime}$ is an open subvariety of a toric variety $\widetilde{Z}_{r}$ with nef anticanonical bundle and birationally proper over $\mathbb{A}^{2}$. In particular, the anticanonical divisor of $\widetilde{Z}_{r}^{\prime}$ is nef. The open subvariety $Z_{r}^{\prime}$ of $Z_{r}$ whose fan is $\Delta_{r} \backslash\left\{\operatorname{cone}\left(e_{1}+r e_{2}, e_{2}\right)\right.$, cone $\left.\left(e_{2}\right)\right\}$ satisfies these properties. So it is sufficient to prove the claim.

We show the claim by induction on $r$. We have already verified the case $r=2$. Let $\widetilde{Z}_{r}^{\prime}$ be a variety that satisfies the hypotheses of the claim and let $\sigma^{\prime}$ be the unique cone in $\widetilde{\Delta}_{r}^{\prime}(2)$ which contains $e_{1}+r e_{2}$. Because of the inductive hypothesis it is sufficient to show that $\sigma^{\prime}=\operatorname{cone}\left(e_{1}+r e_{2}, e_{1}+(r-1) e_{2}\right)$.

Let $k$ be the function associated to the anticanonical bundle of a fixed $\widetilde{Z}_{r}$. Let $k_{\sigma^{\prime}}=x_{1} e_{1}^{*}+x_{2} e_{2}^{*}$. Then $1=k_{\sigma^{\prime}}\left(e_{1}+r e_{2}\right)=x_{1}+r x_{2}$ and $x_{i}=k_{\sigma^{\prime}}\left(e_{i}\right) \leq 1$ for each $i$, so the unique possibilities for $k_{\sigma^{\prime}}$ are $e_{1}^{*}$ and $-(r-1) e_{1}^{*}+e_{2}^{*}$. Write $\sigma^{\prime}=\operatorname{cone}\left(e_{1}+r e_{2}, v\right)$ with $v=c_{1} e_{1}+c_{2} e_{2}$ primitive. If $k_{\sigma^{\prime}}=-(r-1) e_{1}^{*}+e_{2}^{*}$, then $c_{2}=(r-1) c_{1}+1$ because $\left(-(r-1) e_{1}^{*}+e_{2}^{*}\right)(v)=1$. Because of the nonsingularity of $Z$ we have $c_{1}-1= \pm 1$, so there are two possibilities: either $\sigma^{\prime}=$ 
$\operatorname{cone}\left(e_{1}+r e_{2}, e_{2}\right)$ or $\sigma^{\prime}=\operatorname{cone}\left(e_{1}+r e_{2}, 2 e_{1}+(2 r-1) e_{2}\right)$. We exclude the first one because $e_{2}$ does not belong to $\left|\widetilde{\Delta}_{r}^{\prime}\right|$. We also exclude the second one because $k_{\text {cone }\left(e_{1}, e_{1}+e_{2}\right)}\left(2 e_{1}+(2 r-1) e_{2}\right)=2>k(v)$. If $k_{\sigma^{\prime}}=e_{1}^{*}$, then $c_{1}=k_{\sigma^{\prime}}(v)=1$. Because of the smoothness of $Z$ we have $c_{2}-r= \pm 1$. Again, we exclude $e_{1}+(r+1) e_{2}$ because it does not belong to $\left|\widetilde{\Delta}_{r}^{\prime}\right|$. Thus $\sigma^{\prime}=\operatorname{cone}\left(e_{1}+r e_{2}, e_{1}+(r-1) e_{2}\right)$.

Suppose $\Delta_{X}[p]=\left\{e_{1}, e_{1}+e_{2}, e_{1}+2 e_{2}, \ldots, e_{1}+r e_{2}, e_{2}\right\}$. Then $-K_{X}$ is nef if and only if $-2 \delta+2 \delta_{0}+e_{1}^{*}$ and $-2 \delta+2 \delta_{0}-(r-1) e_{1}^{*}+e_{2}^{*}$ are antidominant. In that case, if $\theta$ is decomposable, then the sum of these weights is regular, so $-K_{X}$ is big. If $r=1$ and the previous weights are regular, then $-K_{X}$ is ample. Note that if $r=1$ and $\theta$ is decomposable, such weights are always regular.

(III) If $X$ is not toroidal and $\theta$ is indecomposable, let $X^{\prime}$ be as in Lemma 6.4. By Lemma 6.1 and Theorem 2.6, $R_{G, \theta}=A_{2}, H=G^{\theta}$ and $\Delta_{X^{\prime}}[p]$ contains properly $\left\{\alpha_{1}^{\vee},-\alpha_{1}^{\vee}-\alpha_{2}^{\vee},-3 \omega_{1}^{\vee}\right\}$, up to reindexing. By Lemma 6.5 we have two possibilities for $\Delta_{X^{\prime}}[p]$ : either (i) $\left\{\alpha_{1}^{\vee},-\omega_{1}^{\vee}-\omega_{2}^{\vee},-4 \omega_{1}^{\vee}-\omega_{2}^{\vee},-5 \omega_{1}^{\vee}-2 \omega_{2}^{\vee}, \ldots\right.$, $\left.-(r+3) \omega_{1}^{\vee}-r \omega_{2}^{\vee},-3 \omega_{1}^{\vee}\right\}$ or (ii) $\left\{\alpha_{1}^{\vee},-\omega_{1}^{\vee}-\omega_{2}^{\vee},-4 \omega_{1}^{\vee}-\omega_{2}^{\vee},-7 \omega_{1}^{\vee}-\omega_{2}^{\vee}, \ldots\right.$, $\left.-(3 r+1) \omega_{1}^{\vee}-\omega_{2}^{\vee},-3 \omega_{1}^{\vee}\right\}$.

Recall $a_{i}=\left(2 \delta-2 \delta_{0}\right)\left(\alpha_{i}^{\vee}\right)$. In the first case $k$ is linear over cone $\left(\alpha_{1}^{\vee},-\omega_{1}^{\vee}-\omega_{2}^{\vee}\right)$, $\sigma:=\operatorname{cone}\left(-\omega_{1}^{\vee}-\omega_{2}^{\vee},-(r+3) \omega_{1}^{\vee}-r \omega_{2}^{\vee}\right)$ and cone $\left(-(r+3) \omega_{1}^{\vee}-r \omega_{2}^{\vee},-3 \omega_{1}^{\vee}\right)$. The unique non-trivial condition is $k_{\sigma}\left(\alpha_{1}^{\vee}\right)=r \leq a_{1}$. In the second case $k$ is linear over cone $\left(\omega_{1}^{\vee},-\omega_{1}^{\vee}-\omega_{2}^{\vee}\right)$, cone $\left(-\omega_{1}^{\vee}-\omega_{2}^{\vee},-(3 r+1) \omega_{1}^{\vee}-\omega_{2}^{\vee}\right)$ and $\sigma:=$ cone $\left(-(3 r+1) \omega_{1}^{\vee}-\omega_{2}^{\vee},-3 \omega_{1}^{\vee}\right)$. The unique non-trivial condition is $k_{\sigma}\left(\alpha_{2}^{\vee}\right)=$ $2 r-1 \leq a_{2}$ (or equivalently $\widetilde{k}_{\sigma}\left(\alpha_{2}^{\vee}\right) \leq 0$ ). If $r=1$, then $\theta=-$ id over $\chi_{*}(T)$ so that $-K_{X}$ is not ample.

(IV) Finally, suppose $X$ non-toroidal and $\theta$ decomposable. Suppose also $X_{0}$ is smooth; the case of $X_{0}$ singular is very similar. By the proof of Theorem 2.6, $\rho(D(X))$ cannot be $\rho(D(G / H))$. If $\rho(D(X))=\alpha_{1}^{\vee}$, then, by the local factoriality of $X, e_{1}=-\alpha_{1}^{\vee}$ and there is $r \in \mathbb{Z}^{>0}$ with $\sigma:=\operatorname{cone}\left(\alpha_{1}^{\vee}, v^{\prime}=-r \alpha_{1}^{\vee}+e_{2}\right) \in \Delta$. We apply Lemma 6.4 to the maximal open toroidal subvariety of $X$ (whose colored fan has support cone $\left.\left(-\alpha_{1}^{\vee}, v^{\prime}\right)\right)$. Then, by Lemma $6.5, \Delta[p]$ has to be $\left\{-\alpha_{1}^{\vee}, v^{\prime}\right.$, $\left.-\alpha_{1}^{\vee}+v^{\prime},-\alpha_{1}^{\vee}+2 v^{\prime}, \ldots,-\alpha_{1}^{\vee}+s v^{\prime}, \alpha_{1}^{\vee}\right\},\left\{-\alpha_{1}^{\vee}, v^{\prime},-\alpha_{1}^{\vee}+v^{\prime},-2 \alpha_{1}^{\vee}+v^{\prime}, \ldots\right.$, $\left.-s \alpha_{1}^{\vee}+v^{\prime}, \alpha_{1}^{\vee}\right\}$ or $\left\{-\alpha_{1}^{\vee}, v^{\prime}, \alpha_{1}^{\vee}\right\}$.

In the first case, $k$ is linear over cone $\left(\alpha_{1}^{\vee}, v^{\prime}\right), \sigma_{1}:=\operatorname{cone}\left(v^{\prime},-\alpha_{1}^{\vee}+s v^{\prime}\right)$ and $\sigma_{2}:=\operatorname{cone}\left(-\alpha_{1}^{\vee}+s v^{\prime},-\alpha_{1}^{\vee}\right)$. The non-trivial conditions are $\tilde{k}_{\sigma_{1}}\left(\alpha_{1}^{\vee}\right) \leq 0$ and $\tilde{k}_{\sigma_{2}}\left(\alpha_{2}^{\vee}\right) \leq 0$ (or equivalently $s \leq \bar{m}_{1}+1$ and $r \leq \bar{m}_{2}$ ). If $s=1$, then $r>m_{2}$ because $-K_{X}$ is not ample. In the second case we can suppose $s \geq 2 ; k$ is linear over cone $\left(\alpha_{1}^{\vee}, v^{\prime}\right), \sigma_{1}:=\operatorname{cone}\left(v^{\prime},-s \alpha_{1}^{\vee}+v^{\prime}\right)$ and $\sigma_{2}:=\operatorname{cone}\left(-s \alpha_{1}^{\vee}+v^{\prime},-\alpha_{1}^{\vee}\right)$. The unique non-trivial condition is $\tilde{k}_{\sigma_{2}}\left(\alpha_{2}^{\vee}\right) \leq 0$, or, equivalently, $r+s-1 \leq \bar{m}_{2}$. In the first two cases $\tilde{k}_{\sigma_{1}}\left(\alpha_{2}^{\vee}\right)<0$ and $\tilde{k}_{\sigma_{2}}\left(\alpha_{1}^{\vee}\right)<0$, so $-\widetilde{K}_{X}$ is big. In the last case, we 
proceed as in the proof of Theorem 2.6, obtaining $m_{2}+1<r \leq \bar{m}_{2}+1$. Moreover, $\tilde{k}_{\text {cone }\left(\alpha_{1}^{\vee}, v^{\prime}\right)}\left(\alpha_{2}^{\vee}\right)<0$ and $\tilde{k}_{\text {cone }\left(-\alpha_{1}^{\vee}, v^{\prime}\right)}\left(\alpha_{1}^{\vee}\right)<0$, so $-K_{X}$ is big.

We can study the smoothness of all the previous varieties as in the proof of Theorem 2.6. Note that if $H=G^{\theta}, \theta$ is decomposable, $\sharp \rho^{-1}\left(\alpha_{1}^{\vee}\right)=2$ and $(C(Y), D(Y))=\left(\operatorname{cone}\left(\alpha_{1}^{\vee},-r \alpha_{1}^{\vee}-\alpha_{2}^{\vee}\right), F\right)$, then $Y$ is smooth if and only if $\sharp F=1$ and $\alpha_{1}$ is not exceptional (but $Y$ cannot be an open subvariety of a Fano variety). The symmetric varieties in the statement are all projective. Indeed, if $\Delta_{X}[p]=$ $\left.\left\{e_{1}, e_{1}+e_{2}, e_{1}+2 e_{2}, \ldots, e_{1}+(s-1) e_{2}, e_{1}+s e_{2}, e_{2}\right)\right\}$, then the following divisor is ample: $-p K_{X}+\sum_{i=1}^{s} i^{2} D_{e_{1}+i e_{2}}+m \sum_{D \in D(G / H) \backslash D(X)} D$ with $p, m \gg 0$. Indeed the piecewise linear function associated to $\sum_{i=1}^{s} i^{2} D_{e_{1}+i e_{2}}$ is strictly convex on $\left\{C \in \Delta_{X}: C \subset\right.$ cone $\left.\left(e_{1}, e_{1}+s_{2}\right)\right\}$. Moreover, $k_{X}$ is strictly convex on the fan with maximal cones cone $\left(e_{1}, e_{1}+s_{2}\right)$ and cone $\left(e_{1}+s_{2}, e_{2}\right)$. The other cases are similar.

\section{$\S 6.3$. Symmetric Fano varieties with $G$ reductive}

Finally we classify the 2-rank locally factorial Fano symmetric varieties acted upon by a group $G$ that is only reductive. Recall that $G=G^{\prime} \times \mathbb{C}^{*}, \chi_{*}\left(\mathbb{C}^{*} /\left(\mathbb{C}^{*}\right)^{\theta}\right)=\mathbb{Z} f$ and $e$ is the primitive multiple of $-\alpha^{\vee}$. Observe that $X$ cannot be simple because the valuation cone is not strictly convex (and $N(H) / H$ is not finite). We begin with a lemma similar to Lemma 6.2 .

Lemma 6.6. Let $X$ be a Fano locally factorial symmetric variety with $G$ as before. If $X$ is toroidal, there are at most four colored 1-cones. Otherwise, there are at most three colored 1-cones.

Proof. First suppose $X$ is toroidal. Then $k( \pm f)=1$. Let $\sigma \in \Delta(2)$ be a cone that contains neither $f$ nor $-f$. Then $k_{\sigma}( \pm f) \leq 0$, so $k_{\sigma}$ is a multiple of $e^{*}$. If there is another cone $\sigma^{\prime} \in \Delta(2)$ with the same properties (and such that $\operatorname{dim}\left(\sigma \cap \sigma^{\prime}\right)=1$ ), then $k_{\sigma}=k_{\sigma^{\prime}}$. If $X$ is not toroidal, we can study the maximal open toroidal subvariety $X^{\prime}$ of $X$ as in Lemma 6.2 (because $\left|\mathcal{F}\left(X^{\prime}\right)\right|$ is strictly convex).

Proof of Theorem 2.8. We have to require, as in Theorem 2.6, that $\sharp \rho(D(X))$ $=\sharp \rho^{-1}(\rho(D(X)))$, but in this case $\sharp \rho(D(X)) \leq 1$. If $X$ is not toroidal, then $e=-\alpha^{\vee}$, because there is an appropriate cone $\left(\alpha^{\vee}, v\right)$ in $\Delta(2)$. Suppose first that $\chi_{*}(S)=\mathbb{Z} e \oplus \mathbb{Z} f$. We have to consider the following cases:

1) If $\Delta[p]=\{f,-f, v\}$, then $v=e+r f$ because $\{f, v\}$ is a basis of $\chi_{*}(S)$. We have to impose $\widetilde{k}_{\text {cone }(f, e+r f)}\left(\alpha^{\vee}\right)=\psi(r-1)\left(\alpha^{\vee}\right)<0$ and $\widetilde{k}_{\text {cone }(-f, e+r f)}\left(\alpha^{\vee}\right)=$ $\psi(-r-1)\left(\alpha^{\vee}\right)<0$.

2) Suppose $\Delta[p]=\left\{f,-f, v_{1}, v_{2}\right\}$. As before $v_{i}=e+x_{i} f$; moreover $x_{1}=$ $x_{2} \pm 1$ because $\left\{v_{1}, v_{2}\right\}$ is a basis of $\chi_{*}(S)$. Suppose $x_{1}=x_{2}+1$. We have to 
impose that $\widetilde{k}_{\text {cone }\left(f, e+\left(x_{2}+1\right) f\right)}\left(\alpha^{\vee}\right)=\psi\left(x_{2}\right)\left(\alpha^{\vee}\right)<0$ and $\widetilde{k}_{\text {cone }\left(-f, e+x_{2} f\right)}\left(\alpha^{\vee}\right)=$ $\psi\left(-x_{2}-1\right)\left(\alpha^{\vee}\right)<0$.

3) Suppose $\Delta[p]=\left\{\alpha^{\vee},-f, v\right\}$. Then $v=-\alpha^{\vee}+r f=-m \alpha^{\vee} \pm f$ because $X$ is locally factorial. But $-f$ is not contained in cone $\left(\alpha^{\vee}, v\right)$, thus $v=-\alpha^{\vee}+f$.

4) Suppose $\Delta[p]=\left\{\alpha^{\vee},-f, v_{1}, v_{2}\right\}$ and $\Delta(2)=\left\{\operatorname{cone}\left(\alpha^{\vee}, v_{1}\right), \sigma:=\operatorname{cone}\left(v_{1}\right.\right.$, $\left.v_{2}\right)$, cone $\left.\left(-f, v_{2}\right)\right\}$. Then $v_{1}=-m \alpha^{\vee}+f$ and $v_{2}=-\alpha^{\vee}+r f$. Observe that $\mathbb{R}^{\geq 0} v_{1}=\mathbb{R}^{\geq 0}\left(-\alpha^{\vee}+\frac{1}{m} f\right)$, so $r \leq 0$. Furthermore $v_{2}-r v_{1}=-(1-r m) \alpha^{\vee}= \pm \alpha^{\vee}$, so $m r$ is 0 or 2 . Thus $v_{2}=-\alpha^{\vee}$. Moreover $h_{\sigma}(-f)=m-1<1$, so $v_{1}=-\alpha^{\vee}+f$.

$5)$ Suppose $\Delta[p]=\left\{\alpha^{\vee}, v_{1}, v_{2}\right\}$ and $\Delta(2)=\left\{\operatorname{cone}\left(\alpha^{\vee}, v_{1}\right)\right.$, cone $\left(v_{1}, v_{2}\right)$, $\left.\operatorname{cone}\left(\alpha^{\vee}, v_{2}\right)\right\}$. Thus $v_{1}=-r \alpha^{\vee}+f$ and $v_{2}=-m \alpha^{\vee}-f$ with $r, m>0$. Moreover, $v_{1}+v_{2}=-(r+m) \alpha^{\vee}= \pm \alpha^{\vee}$, a contradiction.

6) Suppose $\Delta[p]=\left\{\alpha^{\vee}, v_{1}, v_{2}, v_{3}\right\}$ and $\Delta(2)=\left\{\operatorname{cone}\left(\alpha^{\vee}, v_{1}\right), \sigma:=\operatorname{cone}\left(v_{1}, v_{2}\right)\right.$, cone $\left(v_{2}, v_{3}\right)$, cone $\left.\left(\alpha^{\vee}, v_{3}\right)\right\}$. Write $v_{2}=-x \alpha^{\vee}+y f$. By the local factoriality of $X$, $v_{1}=-r \alpha^{\vee}+f, v_{3}=-m \alpha^{\vee}-f, x=-m y \pm 1$ and $x=+r y \pm 1$. The last two conditions plus $x>0$ imply that $v_{2}=-\alpha^{\vee}$. Moreover $k_{\sigma}\left(v_{3}\right)=m+r-1<1$, a contradiction.

Now, suppose $\chi_{*}(S)=\mathbb{Z} \frac{f+\alpha^{\vee}}{2} \oplus \mathbb{Z} f$.

1) The toroidal case can be studied as before.

2) Suppose that $\Delta(2)$ contains two cones $\sigma_{ \pm}:=\operatorname{cone}\left(v_{ \pm}, \alpha^{\vee}\right)$. Let $u=\frac{1}{2} \alpha^{\vee}+$ $\frac{1}{2} f$. We have $v_{+}=-\frac{2 m+1}{2} \alpha^{\vee}+\frac{1}{2} f=-(2 m+1) u+(m+1) f$ and $v_{-}=-\frac{2 r+1}{2} \alpha^{\vee}-$ $\frac{1}{2} f=-(2 r+1) u+r f$ with $m, r \geq 0$. First, suppose that there is another $v=-x u+$ $y f$ in $\Delta[p]$; here $x>0$. By the local factoriality we have $x(m+1)=y(2 m+1) \pm 1$ and $x r=y(2 r+1) \pm 1$. Thus $x[(m+1)(2 r+1)-r(2 m+1)]= \pm 1 \mp 1$, so $x(m+r+1)=2, x(m+1)=y(2 m+1)+1$ and $x r=y(2 r+1)-1$. If $x=1$, then the previous three equations are not compatible. Instead, if $x=2$ then $\Delta[p]=$ $\left\{\alpha^{\vee},-\frac{1}{2} \alpha^{\vee}+\frac{1}{2} f,-\alpha^{\vee},-\frac{1}{2} \alpha^{\vee}-\frac{1}{2} f\right\}$. Next, suppose that $\Delta[p]=\left\{\alpha^{\vee}, v_{+}, v_{-}\right\}$; in particular $\left\{v_{+}, v_{-}\right\}$is a basis. Thus $v_{+}+v_{-}=-(m+r+1) \alpha^{\vee}= \pm \alpha^{\vee}$, so $\Delta[p]=\left\{\alpha^{\vee},-\frac{1}{2} \alpha^{\vee}+\frac{1}{2} f,-\frac{1}{2} \alpha^{\vee}-\frac{1}{2} f\right\}$.

3) Finally, suppose that $\Delta[p]$ contains $\alpha^{\vee}$ and $-f$. Then $\Delta$ contains $\sigma=$ $\operatorname{cone}\left(\alpha^{\vee}, v_{1}\right)$ and cone $\left(-f, v_{2}\right)$, with $v_{1}=-\frac{2 m+1}{2} \alpha^{\vee}+\frac{1}{2} f, m \geq 0$ and $v_{2}=-\frac{1}{2} \alpha^{\vee}-$ $\frac{2 r+1}{2} f$. If $v_{1}=v_{2}$, then $\Delta[p]=\left\{\alpha^{\vee},-f,-\frac{1}{2} \alpha^{\vee}+\frac{1}{2} f\right\}$. Otherwise, by Lemma $6.6, \Delta$ contains cone $\left(v_{1}, v_{2}\right)$. Furthermore, $(2 r+1) v_{1}+v_{2}= \pm \alpha^{\vee}$, hence $(2 r+1)(2 m+1)$ is 1 or -3 . But $r \geq 0$ because $v_{2} \notin \sigma$, so $v_{1}=-\frac{1}{2} \alpha^{\vee}+f$ and $v_{2}=-\frac{1}{2} \alpha^{\vee}-f$. The smoothness of these varieties can be studied as in Theorems 2.6 and 2.7.

\section{References}

[A83] D. N. Akhiezer, Equivariant completions of homogeneous algebraic varieties by homogeneous divisor, Ann. Global Anal. Geom. 1 (1983), 49-78. Zbl 0537.14033 MR 0739893 
[AlBr04] V. Alexeev and M. Brion, Boundedness of spherical Fano varieties, in The Fano conference, Univ. Torino, Turin, 2004, 69-80. Zbl 1087.14028 MR 2112568

[Bo68] N. Bourbaki, Éléments de mathématiques. Fasc. XXXIV. Groupes et algèbres de Lie. Chapitres IV, V, VI, Act. Sci. Indust. 1337, Hermann, Paris, 1968. Zbl 0186.33001 MR 0240238

[Br89] M. Brion, Groupe de Picard et nombres caractéristiques des variétés sphériques, Duke Math. J. 58 (1989), 397-424. Zbl 0701.14052 MR 1016427

[Br97a] Variétés sphériques, Notes de cours, 1997.

[Br97b] Curves and divisors in spherical varieties, in Algebraic groups and Lie groups, Austral. Math. Soc. Lect. Ser. 9, Cambridge Univ. Press, 1997, 21-34. Zbl 0883.14024 MR 1635672

[ChMa03] R. Chirivì and A. Maffei, The ring of sections of a complete symmetric variety, J. Algebra 261 (2003), 310-326. Zbl 1055.14052 MR 1966632

[dCoPr83] C. De Concini and C. Procesi, Complete symmetric varieties, in Invariant theory, Lecture Notes in Math. 996, Springer, New York, 1983, 1-44. Zbl 0581.14041 MR 0718125

[dCoPr85]_, Complete symmetric varieties II. Intersection theory, in Algebraic groups and related topics (Kyoto/Nagoya, 1983), Adv. Stud. Pure Math. 6, North-Holland, 1985, 481-513. Zbl 0596.14041 MR 0803344

[Kn91] F. Knop, The Luna-Vust theory of spherical embeddings, in Proceedings of the Hyderabad Conference on Algebraic Groups (Hyderabad, 1989), Manoj Prakashan, Madras, 1991, 225-249. Zbl 0812.20023 MR 1131314

[Ru07] A. Ruzzi, Smooth projective symmetric varieties with Picard number equal to one, Int. J. Math. 22 (2011), 145-177. Zbl 1213.14092 MR 2782684

[Ru09] _ Effective and big divisors on a projective symmetric variety, arXiv:0906.5226.

[Ru10] _ Geometrical description of smooth projective symmetric varieties with Picard number one, Transform. Groups 15 (2010), 201-226. Zbl 1194.14071 MR 2600702

[T06] D. A. Timashev, Homogeneous spaces and equivariant embeddings, Encyclopaedia Math. Sci. 138, Springer, 2011. Zbl pre05844504 MR 2797018

[Vu90] T. Vust, Plongements d'espaces symétriques algébriques: une classification, Ann. Scuola Norm. Sup. Pisa Cl. Sci. (4) 17 (1990), 165-195. Zbl 0728.14041 MR 1076251

[Wa72] G. Warner, Harmonic analysis on semi-simple Lie groups, Vols. I, II, Springer, New York, 1972. Zbl 0265.22020(I) Zbl 0265.22021(II) MR 0498999(I) MR 0499000(II) 